\title{
BUILDING AN IMPROVED PRIMARY HEALTH CARE SYSTEM IN TURKEY THROUGH CARE INTEGRATION
}

\author{
BY \\ SAFIR SUMER \\ JOANNE SHEAR \\ AHMET LEVENT YENER \\ JULY 17, 2019
}

(4) WORLD BANK GROUP

The Government of Japan 
(C) 2019 The World Bank

1818 H Street NW, Washington DC 20433, USA.

Telephone: 202-473-1000; Internet: www.worldbank.org.

\section{Some rights reserved}

The report entitled Building an Improved Primary Health Care System in Turkey through Care Integration has been produced under the grant received from the Japan Policy and Human Resources Development (PHRD) Technical Assistance Program which is financed by the Government of Japan.

This work is a product of the staff and consultants of the World Bank. The findings, interpretations, and conclusions expressed in this work do not necessarily reflect the views of the Executive Directors of The World Bank or the governments they represent. The World Bank does not guarantee the accuracy of the data included in this work.

\section{Rights and Permissions}

The material in this work is subject to copyright. Because The World Bank encourages dissemination of their knowledge, this work may be reproduced, in whole or in part, for noncommercial purposes as long as full attribution to this work is given.

Attribution-Please cite the work as follows: "Sumer, Safir, Joanne Shear, and Ahmet Levent Yener. 2019. Building an Improved Primary Health Care System in Turkey through Care Integration. (c) World Bank."

All queries on rights and licenses, including subsidiary rights, should be addressed to World Bank Publications, The World Bank Group, 1818 H Street NW, Washington, DC 20433, USA; fax: 202-522-2625;

e-mail: pubrights@worldbank.org. 


\section{Table of Contents}

List of Abbreviations. 3

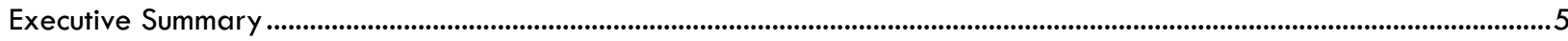

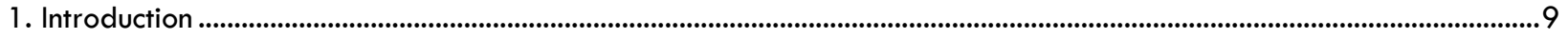

2. Background and Context for Integrated Care in Turkey ….................................................................................................. 11

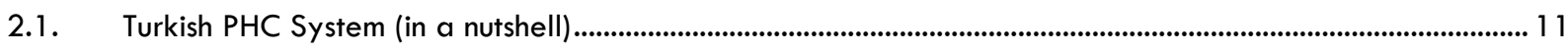

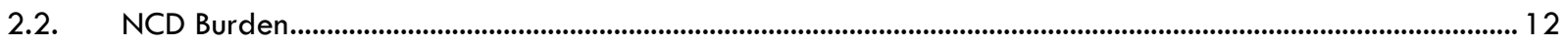

2.3. The Need for Change in Care Delivery Structures ................................................................................................ 15

2.4. Integrated Care at Higher Policy Level in Turkey .................................................................................................. 16

3. Essential Components of Integrated Care: Overview, Country Examples, Status of Turkey, and Recommendations 19

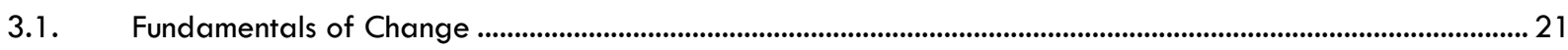

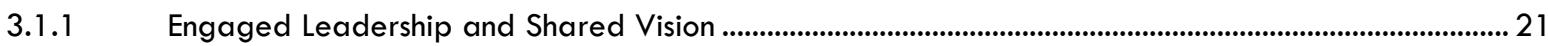

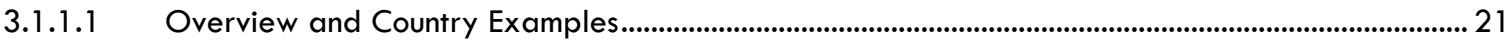

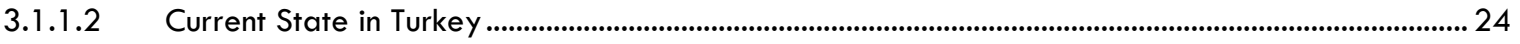

3.1.1.3 Recommendations on Integration within Primary Care Level................................................................25

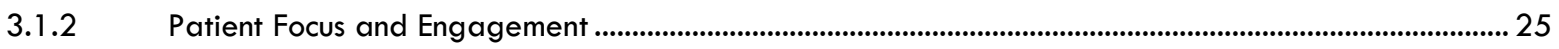

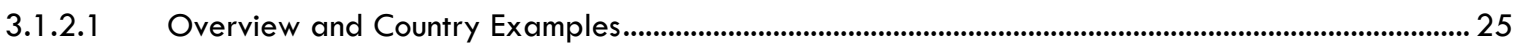

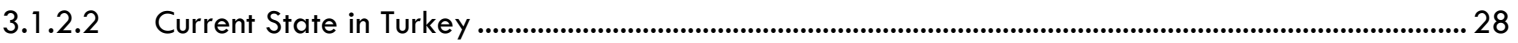

3.1.2.3 Recommendations on Integration within Primary Care Level ..............................................................30

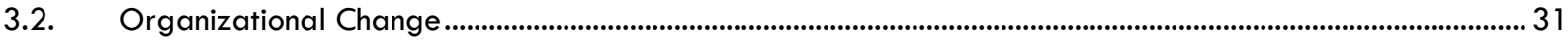

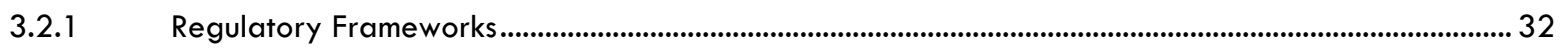

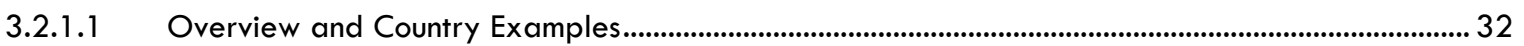

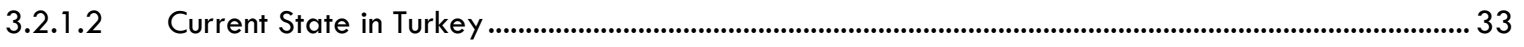

3.2.1.3 Recommendations on Integration within Primary Care Level...............................................................34

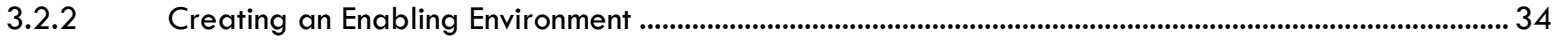

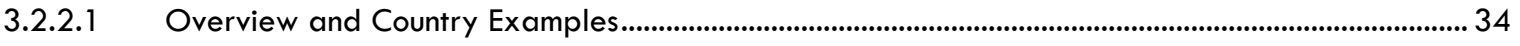

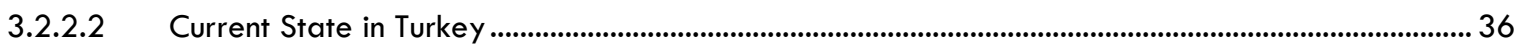

3.2.2.3 Recommendations on Integration within Primary Care Level ..............................................................3.

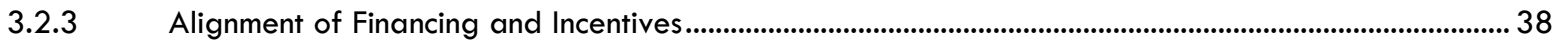

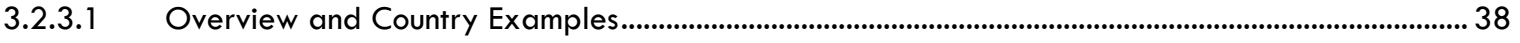

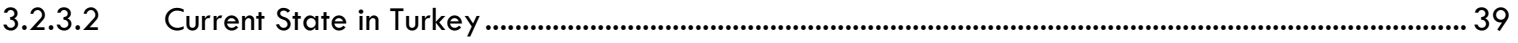

3.2.3.3 Recommendations on Integration within Primary Care Level ............................................................40 


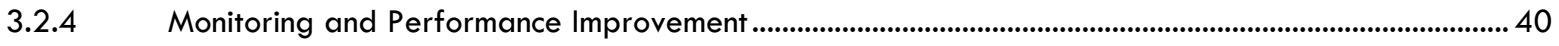

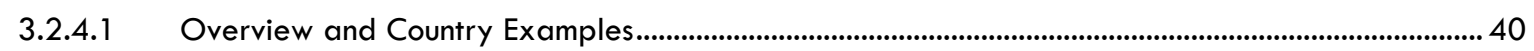

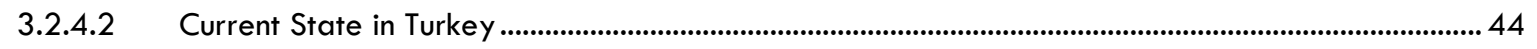

3.2.4.3 Recommendations on Integration within Primary Care Level .............................................................. 44

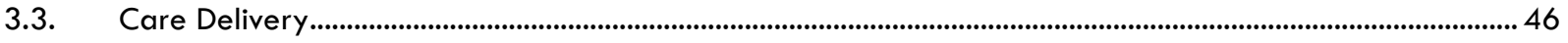

3.3.1 Multidisciplinary Teams and Standardized Training .......................................................................................46

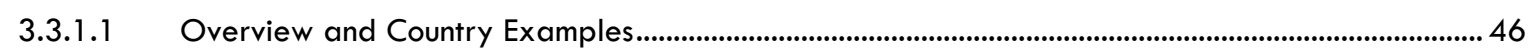

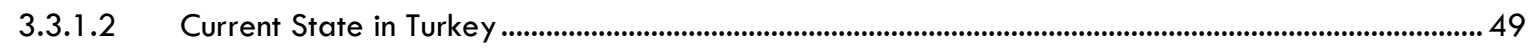

3.3.1.3 Recommendations on Integration within Primary Care Level ................................................................50

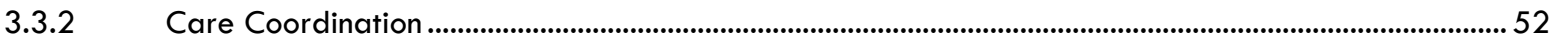

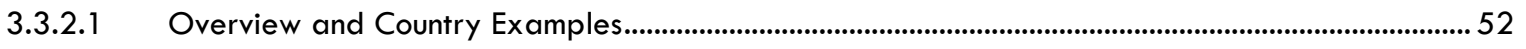

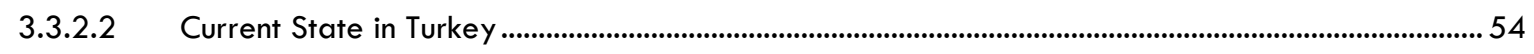

3.3.2.3 Recommendations on Integration within Primary Care Level................................................................5

Evidence-Based Pathways and Protocols ......................................................................................................61

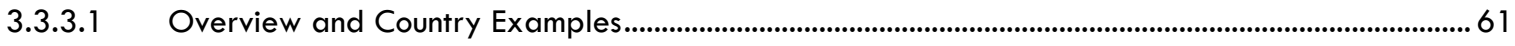

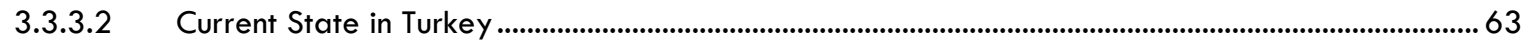

3.3.3.3 Recommendations on Integration within Primary Care Level .............................................................64

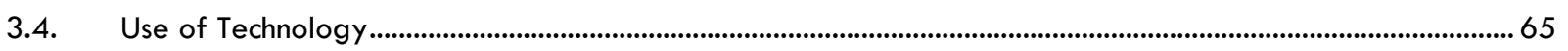

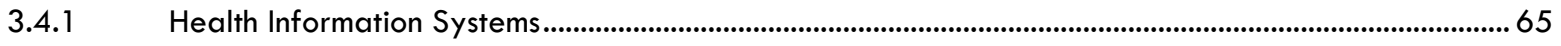

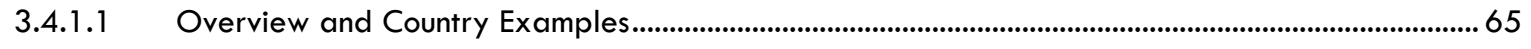

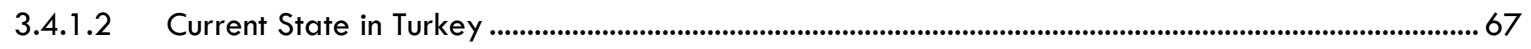

3.4.1.3 Recommendations on Integration within Primary Care Level .............................................................72

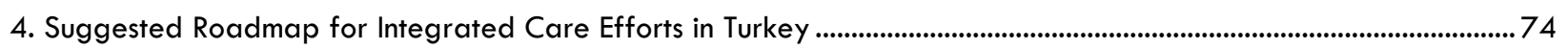

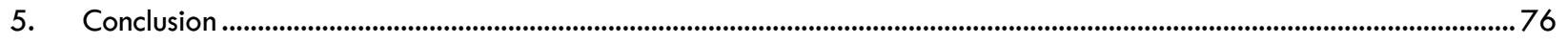

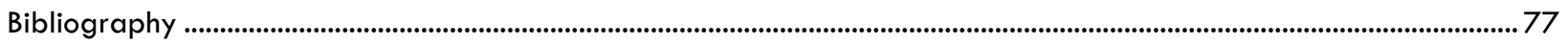

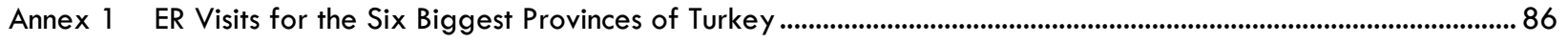

Annex 2 Role of Primary Care Providers in NCD and Elderly Care and Patient Pathways Developed by the MoH

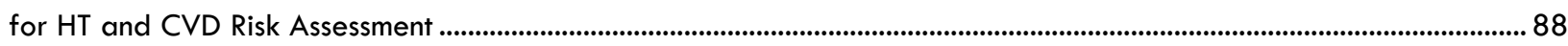


LIST OF ABBREVIATIONS

\begin{tabular}{|c|c|}
\hline ACSC & Ambulatory Care Sensitive Condition \\
\hline AHBS & Family Medicine Information System (Aile Hekimliği Bilgi Sistemi) \\
\hline ARCHO & Assessment of Readiness for Chronicity in Health Care Organizations \\
\hline BI & Business Intelligence \\
\hline CHC & Community Health Center \\
\hline COPD & Chronic Obstructive Pulmonary Disease \\
\hline COSLA & Confederation of Scottish Local Authorities \\
\hline CPG & Clinical Practice Guideline \\
\hline CVD & Cardiovascular Disease \\
\hline DALY & Disability Adjusted Life Year \\
\hline DHBS & Dental Health Information System \\
\hline DM & Diabetes Mellitus \\
\hline DSS & Decision Support System(s) \\
\hline DYOB & Dialysis Management Information System (Diyaliz Bilgi Yönetim Sistemi) \\
\hline EBYS & Electronic Document Management System \\
\hline ED & Emergency Department \\
\hline EHR & Electronic Health Record \\
\hline ER & Emergency Room \\
\hline EU & European Union \\
\hline FM & Family Medicine \\
\hline FMC & Family Medicine Center \\
\hline FMP & Family Medicine Physician \\
\hline GDHIS & General Directorate of Health Information System(s) \\
\hline HbA1c & Hemoglobin A1c \\
\hline HBYS & Hospital Information System (Hastane Bilgi Yönetim Sistemi) \\
\hline HCP & Health and Care Plan \\
\hline HIS & Health Information System(s) \\
\hline HLC & Healthy Living Center \\
\hline
\end{tabular}




\begin{tabular}{|c|c|}
\hline HSYS & Public Health Information System (Halk Sağlığı Yönetim Sistemi) \\
\hline HT & Hypertension \\
\hline HTP & Health Transformation Program \\
\hline HYP & Disease Management Platform (Hastalık Yönetim Platformu) \\
\hline ICD 10 & International Statistical Classification of Diseases and Related Health Problems 10TH Revision \\
\hline ICU & Intensive Care Unit \\
\hline IHI & Institute for Healthcare Improvement \\
\hline IRR & Internal Rate of Return \\
\hline ISBS & Occupational Medicine Information System (İşyeri Hekimliği Bilgi Sistemi) \\
\hline MBYS & Medical Examination Information System (Muayene Bilgi Yönetim Sistemi) \\
\hline MCH & Maternal and Child Health \\
\hline МoH & Ministry of Health \\
\hline MSSQL & Microsoft SQL Server \\
\hline NCD & Noncommunicable Disease \\
\hline NHS & National Health Service \\
\hline NPO & Nonprofit Organization \\
\hline PHC & Primary Health Care \\
\hline PHRD & Japan Policy and Human Resources Development Fund \\
\hline ROI & Return on Investment \\
\hline SDGs & Sustainable Development Goals \\
\hline SINA & Statistical and Causal Analysis in Health \\
\hline SQL & Structured Query Language \\
\hline STEPS & WHO STEPwise Approach to Adult Risk Factor Surveillance \\
\hline TURKSTAT & Turkish Statistical Institute \\
\hline UHTS & National Patient Tracking System (Ulusal Hastalık Takip Sistemi) \\
\hline VHA & Veterans Health Administration, United States \\
\hline WHO & World Health Organization \\
\hline
\end{tabular}




\section{EXECUTIVE SUMMARY}

Turkey has realized a very successful health reform between 2003 and 2013. The Ministry of Health (MoH) has developed strategic objectives and major transformation processes under the reform primarily focused on people and gave priority to improve and reshape the primary care structure through the introduction of the Family Medicine (FM) model. Universal health coverage has been established, and the MoH initiated various programs to improve the service provision at all stages. While the overall transformation process includes many aspects of a broader integration of care, an explicit 'integrated care' model has never been discussed in Turkey. This report therefore aims to provide a picture of the Turkish health system from an integrated care perspective while prioritizing noncommunicable diseases (NCDs) and to inform policy makers and practitioners on the needs and opportunities to design a broader care integration model as well as to present specific recommendations on how to improve integration among the primary health care (PHC) actors.

The scope of this report covers analysis and means for integration within the primary care levels as this seems a good entry point for integrated care within the Turkish context. Discussions on the vertical integration between preventive, primary, and secondary care as well as the financial models for integration are left to future activities/efforts.

Key elements of integrated care, as elaborated in the report, can be grouped under four dimensional areas: fundamentals of change, organizational change, care delivery, and the use of technology (Figure 1).

\section{Figure 1}

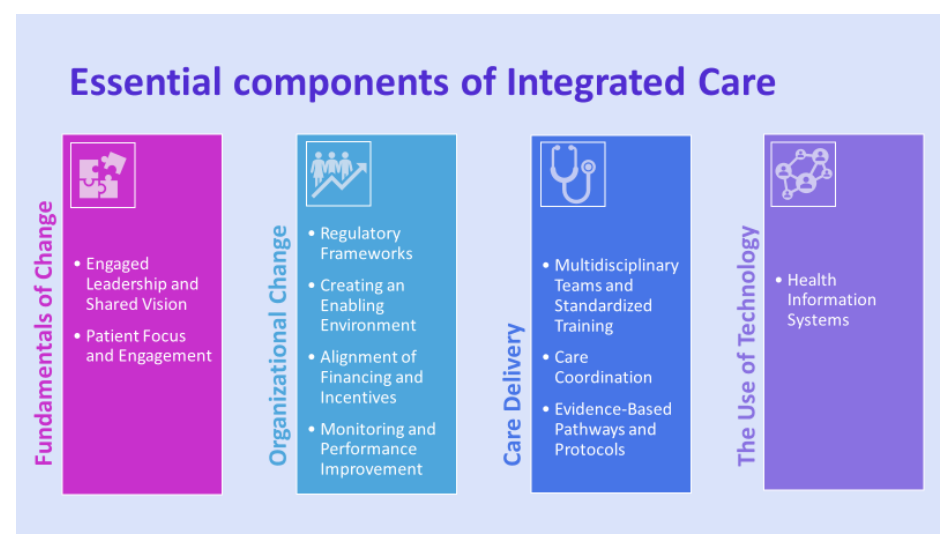

Engaged leadership necessitates charting the course for change and supporting efforts for transformation. Engaged leaders provide direction and motivation and allocate resources through effective strategies. A shared vision at all levels of the transformation process is also critical for successful change within a health care system. Leadership is crucial for articulating a shared vision and conveying the message on the urgency and importance of that vision to all stakeholders. Therefore, leaders need to provide a supportive organizational culture by encouraging open and honest feedback from key stakeholders, which will facilitate transparent monitoring and evaluation and shared decision making to foster public trust.

Patient focus, patient centeredness, and patient engagement and activation are not only essential elements of integrated care models, but they also relate to a number of positive results such as improved health outcomes, improved communication and interactions within the health care team, and patient satisfaction. The concepts of self-care, co-ownership of health care delivery options, discussion on planned outcomes, and informed expression of choice enhance the responsibility and accountability of health care seekers and necessitate revisiting professional identity and scope of responsibilities of the health care workers. Population 
risk stratification based on care needs is another necessity to allocate resources and to plan personalized and patient-centered care delivery.

A review of existing national health policies and regulatory frameworks is important for evaluating progress toward integrated care. While the current approach to addressing chronic diseases is mostly hospital based and has the potential to weaken links of NCDs with population health and wider determinants of health, traditional approaches need to be revisited to shift the burden of care to lower levels of care. Therefore, integrated care introduces new models of care delivery which facilitate this shift toward population health management and proactive and preventive care.

An enabling environment should primarily bring together all involved stakeholders for a transformational change. A cultural change in the clinical and management practices is needed to establish a shared vision and ensure sustainability. To ensure alignment among all the processes and actors involved, top management's strong and visible support for the integrated care efforts and the existence of joint decision-making mechanisms are important. Another enabler is the existence of clinical leadership to communicate a clear vision on how integrated care can produce desired improvements. Lastly, the existence of shared accountability through reliable information sharing is essential since integrated care delivery necessitates the existence and acceptance of clearly defined shared values. ${ }^{1}$

Alignment of finances and incentives, either monetary or non-monetary, can be effective in promoting change. Financing and incentives options are varied, including investments, making the business case, reward schemes, pooled budget, shared risks and revenue/profits, bundled payments, capitated payments, long-term contracts, and end-to-end managed care. While provider payments should generally be designed to encourage performance improvement for providers to improve the experience of care, improve population health status, and reduce costs, incentives and payment methods need to be aligned with broader system goals, to be consistent across providers/payers and to address provider protection from unavoidable risk as well as variation in patient morbidity.

Monitoring and performance improvement through quantitative and qualitative data against baseline is critical in implementing integrated care. Performance indicators should include measurement in clinical quality, patient experience, staff experience, staff satisfaction, and cost effectiveness.

The need for multidisciplinary teams emerges with the transformation of health care systems where new roles need to be created and new skills need to be developed. Additionally, health care demand also changes over time and this calls for retraining the skills and talent and expanding experience through novel practices of care as well as improving the systems to become 'learning systems' that would continuously improve productivity and increase success. As PHC provides the basis for a stable, cost-effective, and efficient health care delivery system, building effective health care teams for an assigned group of patients to provide preventive, chronic, and acute care would mean good PHC practice. Shifting care from hospitals to primary care needs careful planning and execution to ensure that PHC services have the capacity and capabilities to meet the demand. A team-based care approach requires that team members are trained and supported to function effectively, and they are provided with the tools and guidance to effectively deliver care.

Care coordination is the organization of health care delivery activities between multiple providers and the patient to facilitate appropriate health care. Care coordination involves the mobilization of personnel and other resources needed for all required patient care activities and managed by the exchange of information among different providers and the patient. Effective care coordination requires building relationships between PHC

${ }^{1}$ Maruthappu, Hasan, and Zeltner 2015. 
teams, key specialist groups, hospitals, and community service agencies through tools such as referral and transition protocols and systems to support information transfer.

Evidence-based pathways and protocols contribute to the advancement of evidence-based health care and practice with a number of emerging factors such as cost pressures, technological achievements, changing public expectations, value-for-money movement, political consensus, professional accountability, and changing demographic profile. The use of evidence-based pathways and protocols is currently considered a value-added approach to improve patient outcomes and bring efficiency to multidisciplinary work for standardized care and work processes.

Health information systems (HIS) are critical for efficient collection of health system and patient data and for the best use of these data to establish/improve the coordination of patient-provider and intra-provider clinical activities. HIS also concentrate on the use of data for research, planning, and management decisions about integrated care. Health care providers continuously need patient/beneficiary information in terms of their wants and needs and other providers' care details to adjust or to complement the ongoing patient care scheme, and achieving efficient integrated care is possible only with effective communication and information. Therefore, HIS have a critical role in care quality improvement, cost reduction, and establishing of innovative integrated care models. ${ }^{2}$

In light of the essential elements and good country practices of integrated care, a quick look at the Turkish health system reveals several challenges such as the lack of concretely defined objectives for integrated care, limitations to access patient data at all levels of care to plan for integrated care, and lack of adequate health care personnel at the primary care level to address the working modality change that would come with integrated care. In addition, there is lack of financial models/mechanisms to deal with NCDs at the primary care and prominent changes in the $\mathrm{MoH}$ upper management level during the last few years, which make long-term program planning and implementation difficult as there is discontinuous top-level engagement to commit and engage for possible integrated care initiatives.

Nevertheless, there are a number of good practices at both the central and local levels on differing scales, as the report points out. To name a few, prevailing practices include the existence of well-advanced and flexible HIS, small-scale care coordination efforts for a number of diseases, and initial local attempts to draft/reshape care coordination processes as well as regular integrated care data collection procedures.

To support both the good country practices and efforts toward integrated care, this report provides a set of recommendations for integrated care in Turkey based on the essential elements discussed above (Figures 2, 3, 4 , and 5).

These recommendations, together with the country-specific good practice examples, can inspire and leverage the future design efforts of integrated care and if successful, can lead the way to scale up integrated care efforts throughout the health system.

\footnotetext{
2 Øvretveit 2017.
} 
Figure 2

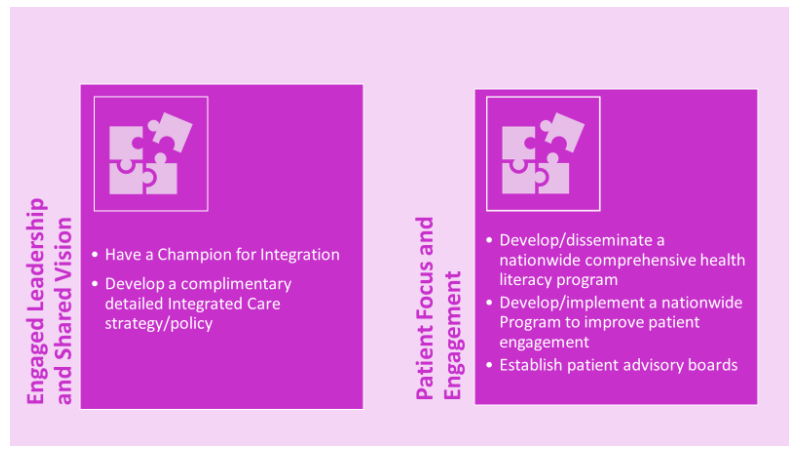

Figure 4

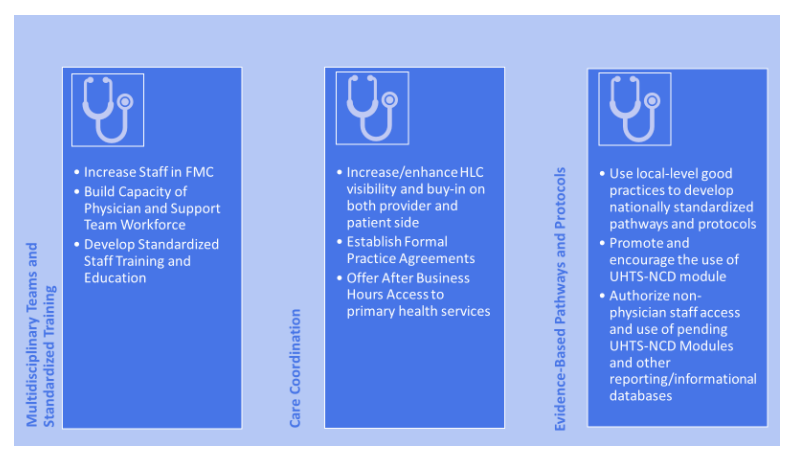

Figure 3

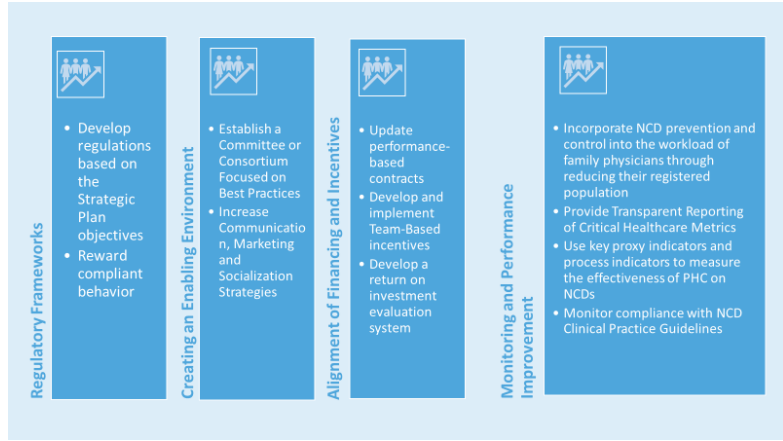

Figure 5

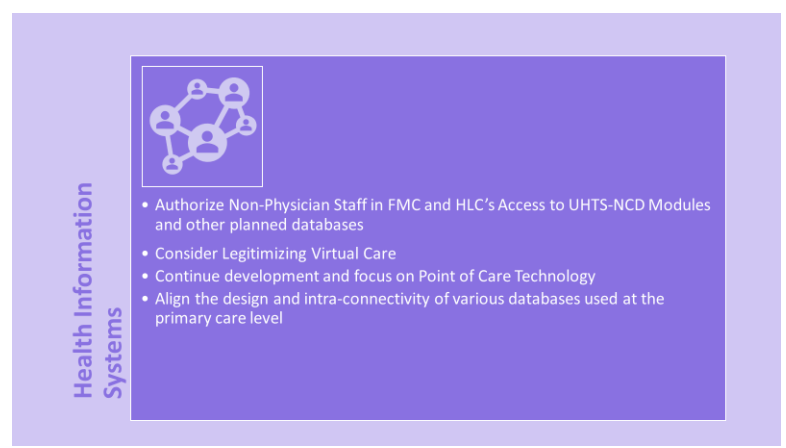




\section{INTRODUCTION}

Sustainable Development Goals (SDGs) adopted by the United Nations member states in 2015 is an international pledge for the current and future well-being of people to end/curb poverty through the reductions in inequality and improvements in areas such as health and education. Goal 3 of the SDGs focuses on ensuring healthy lives and promoting well-being for all at all ages ${ }^{3}$ and calls for increased efforts on health system strengthening to address new and changing health challenges, increase access to health care, and shift the perspective and focus toward meeting the needs of the people served by the health care system. To this end, the notion of integrated and people-centered health care presents a transformation in the management, funding, and delivery of health care services and emphasizes a structural shift from health systems designed around diseases to systems designed for people. ${ }^{4}$

With the increasing pressures of health systems posed on governments, no single actor alone can successfully improve the population's health. All involved in the health system, including health care organizations, public health departments, social service entities, school systems, and employers, need to cooperate. As this cooperation requires an integrator, policy makers can assume this role through making the right investments and creating a clear set of high-level measures to monitor progress. ${ }^{5}$

Improving population health outcomes and the well-being of the populace is an imperative that is shared globally. Over the last 10 years, the international health care community has recognized that current fragmented health care delivery and provider systems hinder good patient access, do not provide continuity of care, are cumbersome for patients to navigate, are inefficient in terms of time and resources, and do not promote and sustain quality health care. A promising model known as 'integrated care' is being widely implemented and studied to address current health care delivery. Integrated care seeks to improve outcomes of care by overcoming issues of fragmentation through linking or coordination of services of providers along the continuum of care. Strong primary care is a key component of integration and continuity between and across levels of care, which is essential for patients, particularly those with complex needs. Primary care can contribute to strengthening the overall health system's performance by providing affordable and accessible care, coordinating care for patients so that they are given the most appropriate services in the right setting, and reducing avoidable hospital admissions. ${ }^{6}$

Health care systems implementing an integrated care approach have achieved reductions in chronic disease morbidity and mortality as well as improvements in prevention care (for example, immunizations) and early detection of cancers and chronic diseases resulting in a decreased disease burden impact on systems and the population. ${ }^{7}$ Integrated care has also demonstrated reductions in the economic impact of ill health and diseases on the government, health care systems, and the people. ${ }^{8}$ A large study conducted in Switzerland demonstrated

3 https://sustainabledevelopment.un.org/sdg3 (accessed December 26, 2018).

4 https://www.who.int/servicedeliverysafety/areas/people-centred-care/en/( (accessed December 26, 2018).

${ }^{5}$ Borgermans and Devroey 2017.

${ }^{6}$ European Union 2018.

7 European Union 2017.

${ }^{8}$ Lyngsø et al. 2014. 
substantial effects of integrated care on reducing medical expenditures (up to 10 percent) and disease-related hospitalizations reflecting the quality of care in patients suffering from highly prevalent chronic diseases. ${ }^{9}$

Integrated care also seeks to improve patient experience through systems redesign that improve care coordination among providers and focuses on service delivery from a consumer's perspective (also known as person or people centered). There is substantial evidence that patient experience and satisfaction with the health care system improve when concepts of integrated care are implemented. ${ }^{10}$

When these factors are taken into consideration, it is clear that integrated care is a comprehensive and highly complex approach to redesign the health care system. Policy makers therefore need to pursue a comprehensive and system-based approach with an emphasis on prevention, effective management of multiple diseases, and multidisciplinary work. From a broader context, policy makers should consider the need to shift funding from individual service providers and institutions to networks of organizations with shared goals as integrated care systems focus mostly on constructs such as patient-centered care, care coordination, continuity of care, chronic disease management, and integrated health care delivery. ${ }^{11}$

This report has been drafted within the scope of the Japan Policy and Human Resources Development Fund (PHRD) Grant focusing on the sustainability of universal health coverage in Turkey. It is expected that the report supports diagnoses of health policies to provide options that will enable the Turkish government to identify appropriate policy and regulatory reform measures needed to achieve inclusive and sustainable expansion in health coverage under the second phase of the Health Transformation Program (HTP).

The main focus of the report is integration at the primary health care (PHC)level. PHC is elaborated for a number of reasons. First, PHC and public health can be considered as the core of integrated health services, ${ }^{12}$ where they address the needs of both the population and individuals, as services complementing each other. Second, as an ultimate goal of health systems as well as of the integrated care practices, primary care can be a good means to achieve better equity for health care and overall improvement in health ${ }^{13}$ by focusing on all health conditions through a number of services. Third, PHC seems to be an effective starting point for integrating health care services in the Turkish context: the Family Medicine (FM) model was introduced about a decade ago in Turkey within the scope of the Turkish HTP. The model has been recently supported with the newly introduced Healthy Living Center (HLC) structures which operate on a multidisciplinary basis and primarily address chronic and long-terms conditions. Effective integration practices among these primary care providers is highly needed to achieve better health conditions.

The report aims to give a general framework for integrated care, to take a broad picture of the Turkish health system from the integrated care building blocks perspective with a particular emphasis on NCDs and PHC and take stock of current strengths, capabilities, and good practices as well as challenges that need to be addressed. A series of in-depth literature surveys and desk studies as well as key informant interviews in the relevant departments of the Ministry of Health $(\mathrm{MoH})$ and field visits to different provinces of Turkey reveal the progress for integrated care and room for improvement, concerning the implementation in the field as well as

\footnotetext{
${ }^{9}$ Huber et al. 2016.

10 WHO 2015.

11 Borgermans and Devroey 2017.

12 https://www.who.int/docs/default-source/primary-health/vision.pdf.

13 http://www.euro.who.int/ data/assets/pdf file/0006/96468/E91878.pdf.
} 
the system and policy design efforts at the central level. The findings from the visits and interviews, together with the country examples, lead the reader to the recommendations and road map sections, where the report intends to provide insight to Turkish decision/policy makers in their efforts toward integrated care.

Section 2 of the report provides an overview of the Turkish context together with the status of background factors necessitating integrated care. Section 3 presents the essential elements of the integrated care according to published literature. It also explores various country examples and Turkey's corresponding state for each of these elements and provides recommendations for efforts toward integrated care in Turkey. Section 4 includes a road map which summarizes the recommendations with their priority levels and timeline for implementation.

\section{BACKGROUND AND CONTEXT FOR INTEGRATED CARE IN TURKEY}

Similar to global trends-changing disease patterns from communicable to noncommunicable diseases-the need to revisit care delivery structures together with the diverging requirements of dealing with NCDs and shifting of the burden of work toward primary care and thus toward prevention, early detection, and control are the main factors that necessitate considering integrating care in Turkish health system.

\subsection{TURKISH PHC SYSTEM (IN A NUTSHELL)}

The first phase of the HTPs was implemented between 2003 and 2013 with the aim of improving access, efficiency, and quality in health care. Primary care has been reformed through the creation of a new primary care specialty and service delivery approach through the FM model, bringing family physicians' salaries up to those of specialists, promoting/encouraging the use of clinical guidelines, implementing well-functioning health information and decision support systems (DSS), and designing properly aligned financial incentives. The FM program was initially introduced as a pilot in 2005 and has been scaled up nationwide in 2010.

Primary care services are delivered in Family Medicine Centers (FMCs) by FM practitioners. FMCs are run by FM practitioners and support staff. As of December 31, 2018, there are 26,252 FM units. The population covered is 3,098 per active FM unit and 3,378 per active FM practitioner. ${ }^{14}$

Family Medicine Physicians (FMPs) and other clinical staff operate under performance-based contracts. They are paid in accordance with a formula based on (a) the population served; (b) socioeconomic development index of the province; (c) costs of mobile health services, examinations, and consumables; and (d) FMC operating costs.

Performance-based contracts are linked with a so-called negative incentive under which up to 20 percent of the providers' payments are withheld if performance targets for maternal and child health (MCH), including vaccinations, are not met.

The FM system does not play a gatekeeper role for the secondary- and tertiary-level care. In addition to FM units, community health centers (CHCs) in the districts deliver community and public health services, including environmental health, reproductive health, child and adolescent health services, communicable disease management and control, and cancer screening.

The FM system is at the center of the PHC provision in Turkey. HLCs have been recently introduced to complement and strengthen the FM model. The conceptual framework for HLCs has been developed by the $\mathrm{MoH}$, including the types of HLCs, general roles and functions, multidisciplinary services to be provided, staffing

14 Ministry of Health Public Health General Directorate, Department of Family Medicine. 
composition, and infrastructure requirements. HLCs have a complementary role within the PHC structure and they support the FM model. HLCs mainly have two objectives. First, they aim to conduct patient follow-up activities when referred by FMCs. Second, they aim to carry out population screening programs to identify and stratify the population for specific diseases (especially for NCDs) and thus be proactive and plan for NCD management. The need for more effective approaches concerning NCD management, expanded target population screening for priority NCDs, and a proper process design for NCD screening, diagnosis, and management has been highlighted in the recent MoH Strategic Plan of 2019-2023 and HLCs aim to complement the FM model in that sense.

There are no standard or widely used clinical pathways in Turkey to deal with NCDs. While MCH is a part of the performance-based contracts at the primary care level, NCDs are still not linked with such contracts in the preventive care programs.

\subsection{NCD BURDEN}

NCDs and their burden are on the rise globally and Turkey is no exception. In 2016, 71.3 percent of all deaths worldwide were due to NCDs. ${ }^{15}$ Among those NCD deaths, 72.9 percent occurred in lower and upper-middle income countries. ${ }^{16}$

The Burden of Disease study conducted by the $\mathrm{MoH}$ in 2004 highlights the changing disease patterns in the country toward chronic diseases. The study suggests that 79 percent of the deaths are due to NCDs. ${ }^{17}$ According to the Turkish Statistical Institute (TURKSTAT) 2018 statistics, cardiovascular diseases (CVDs) and cancers are the top two causes of mortality for the entire population (CVD 38.4 percent and cancer 19.7 percent for the overall population). ${ }^{18}$ The 2004 Burden of Disease study also suggests that NCDs have a substantial share within the top 10 causes of death: ischemic heart disease ranks first - 21.7 percent, cerebrovascular disease ranks second - 15.0 percent, chronic obstructive pulmonary disease (COPD) ranks third - 5.8 percent, hypertensive heart disease ranks sixth - 3.0 percent, lung cancer ranks seventh - 2.7 percent, and diabetes mellitus (DM) ranks eighth - 2.2. Recent World Health Organization (WHO) estimates suggest that NCDs account for 89 percent of all deaths in Turkey, where CVDs account for 34 percent, cancers 23 percent, and diabetes 5 percent. ${ }^{19}$

The Institute for Health Metrics and Evaluation (IHME) estimates also highlight the significant share of NCDs for mortality where 8 out of the top 10 causes of death are due to NCDs. Additionally, IHME figures reveal the concerning increase in mortality due to NCDs between 2007 and 2017 (mortality increase: 44.0 percent hypertensive heart disease, 32.4 percent - ischemic heart disease, 41.5 percent - COPD, and 11.0 percent diabetes). ${ }^{20}$

\footnotetext{
15 http://www.who.int/healthinfo/global burden disease/estimates/en/.

16 http://www.who.int/healthinfo/global_burden_disease/estimates/en/.
}

The classification is the World Bank income groups. As of July 2017, middle-income countries (MICs) are nations with a per capita gross national income (GNI) between US\$1,005 and US\$12,235.

17 https://sbu.saglik.gov.tr/Ekutuphane/Yayin/166 (accessed July 28, .2019).

18 Ölüm Nedeni İstatistikleri, 2018 TUIK http://www.tuik.gov.tr/PreHaberBultenleri.do?id=30626 (accessed June 14, 2019).

19 http://www.who.int/nmh/countries/tur en.pdf?ua=1 (accessed October 10, 2018).

20 http://www.healthdata.org/turkey. 
The WHO's 2014 NCD Turkey country report ${ }^{21}$ suggests that the share of NCD burden in terms of disability adjusted life years (DALYs) increased from 71 percent in 2002 to 79 percent in 2012. In terms of the number of NCD cases, recent data from the MoH General Directorate of Health Information Systems (GDHIS) are consistent with the discussion above and show a remarkable rise in the total number of cases for the most common NCDs, namely diabetes, hypertension (HT), and obesity (Figures 6 and 7). ${ }^{22}$

Changing disease patterns not only have implications on the health economy as losses due to morbidity and mortality, but they require different perspectives to be brought in the health service delivery structures since NCDs are chronic and require long periods of continuous care.

Figure 6

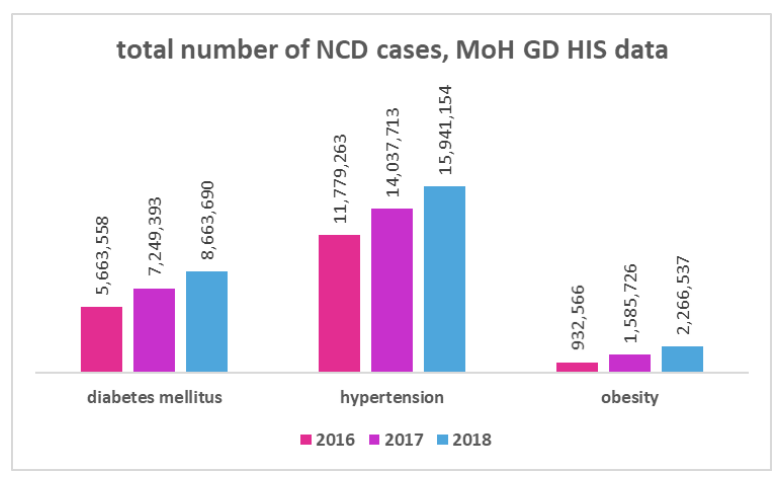

\section{Figure 7}

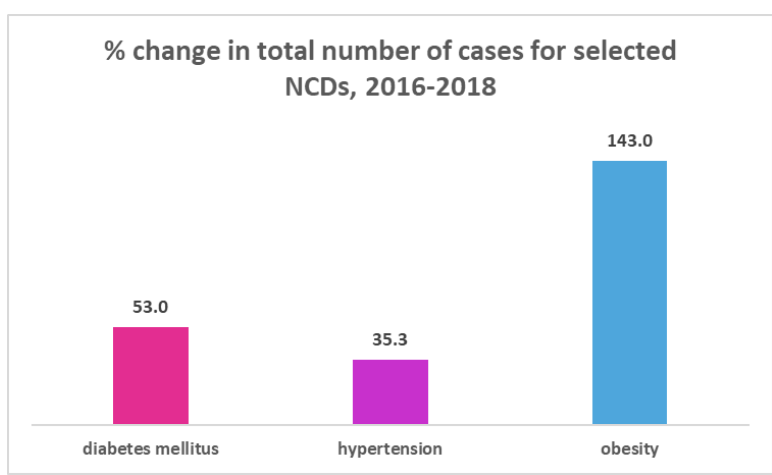

Turkey is experiencing a shift in population demographics toward increasing age. The share of people ages 65 years and above within the total population has increased significantly. The share of people younger than 15 years was 39 percent in 1980 and this number has reduced to 26 percent in 2008. 23 The TURKSTAT recent data also support the decline in the share of the young population and rise in the share of the older population (Figure 8).24

\section{Figure 8}

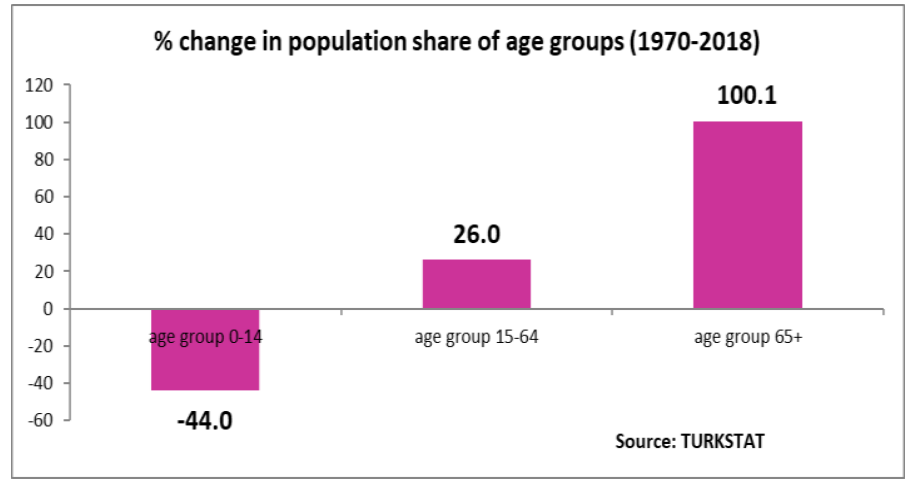

21 https://sbu.saglik.gov.tr/Ekutuphane/kitaplar/BOH \%C3\%9Clke raporu TR.pdf.

22 (a) Figures are based on data collected through e-pulse since 2015 and (b) data from all health institutions are included.

23 http://www.hips.hacettepe.edu.tr/TurkiyeninDemografikDonusumu 220410.pdf (accessed December 22, 2018).

24 http://www.tuik.gov.tr/UstMenu.do? metod=temelist. 
Changing lifestyle and rapid urbanization also exacerbate the situation where the population's exposure to NCD risk factors is high. A recent survey in Turkey ${ }^{25}$ suggests high levels of unhealthy behavior, including dietary habits and physical activity, as well as worrying figures for overweight, obesity, and high blood pressure and glucose levels. Almost 90 percent of the respondents have inadequate fruit and vegetable intake daily, 64 percent are overweight, 29 percent are obese (with a significantly high share for women), almost 30 percent of the respondents have high blood pressure, and 11.5 percent have high blood glucose (Figure 9). Almost half of the respondents have low levels of physical activity; the inactivity levels for women is very high compared to men (61.1 percent for women versus 37.4 percent for men) (Figure 10).

Figure 9

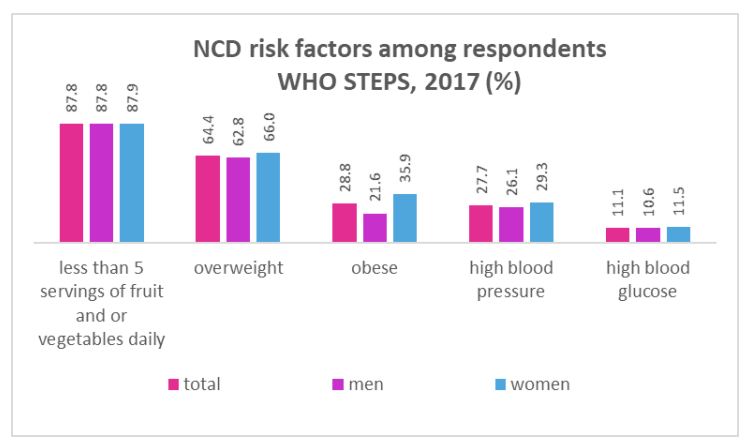

Figure 10

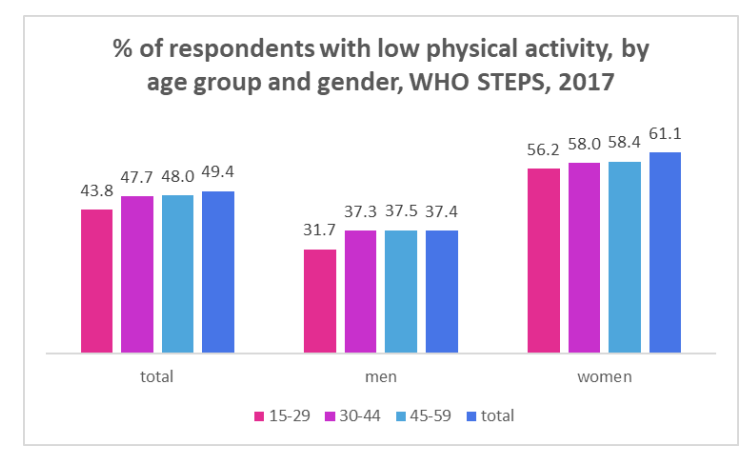

The MoH Department of Healthy Nutrition and Physical Activity indicates that salt intake is high in Turkey (twice as much as the WHO's recommendation ${ }^{26}$ ), although it has reduced from 15.0 grams per day in $2012^{27}$ to 9.9 grams per day in 2017.28 The Department of Healthy Nutrition and Physical Activity also highlights that obesity and diabetes are two important conditions ${ }^{29}$ to focus on in Turkey, where the $\mathrm{MoH}$ aims to stop the increasing trend for these conditions, in line with the WHO 2025 goals. ${ }^{30}$ The department indicates that halting the increasing trend for obesity prevalence from 30.0 percent ${ }^{31}$ and diabetes prevalence from 13.7 percent ${ }^{32}$ has started. There is also progress in the 2025 target of reducing the physically inactive population by 10.0 percent; this figure reduced from 56.0 percent in 2010 to 43.6 percent in 2017.

25 http://www.who.int/ncds/surveillance/steps/WHO Turkey Risk Factors A4 ENG 19.06.2018.pdf (STEPS 2017).

26 https://www.who.int/news-room/fact-sheets/detail/salt-reduction.

27 https:/ www.tdd.org.tr/wp-content/uploads/2018/04/t rkiye a r tuz t ketiminin azalt lmas program_2017-20211.pdf (SALTURK-2).

${ }^{28}$ National Household Health Survey in Turkey 2017,

http://www.euro.who.int/_data/assets/pdf file/0008/383984/turkey-risk-factors-eng.pdf?ua=1.

https://www.tuseb.gov.tr/enstitu/tacese/yuklemeler/ekitap/UA kuruluslar/WHO/DSO Turkiye Steps 2017.pdf (Türkiye Hanehalkı Sağlık Araştırması 2017).

29 Obesity prevalence is 30 percent according to the Turkey Nutritional Health Survey of 2010 and diabetes prevalence is 13.7 percent according to the TURDEP II .(Türkiye Diyabet, Hipertansiyon, Obezite ve Endokrinolojik Hastalıklar Prevalans Çalışması, Turkey Diabetes, Hypertension, Obesity and Endocrinological Diseases Study) study.

30 https://www.who.int/beat-ncds/take-action/targets/en/.

31 https://hsgm.saglik.gov.tr/depo/birimler/saglikli-beslenme-hareketli-hayat-db/Yayinlar/kitaplar/digerkitaplar/TBSA-Beslenme-Yayini.pdf (TBSA-2010).

32 http://www.diabetcemiyeti.org/c/turdep-2-sonuclarinin-ozeti (TURDEP-2-2009). 


\subsection{THE NEED FOR CHANGE IN CARE DELIVERY STRUCTURES}

The rise of NCDs on a global scale calls for rethinking the health care delivery structures which are mainly based on the diagnosis and treatment of acute conditions and finding novel ways to let the system evolve beyond the acute care model which would effectively incorporate the notion of health care delivery for longterm health problems. ${ }^{33}$ To this end, the concept of 'integrated care' is drawing interest for better and more cost-effective health systems, and it is seen as a demand-driven response to the challenges of changing demographics, increased NCD burden, and pressures to reduce costs on avoidable hospitalizations and services that modern health care systems face. ${ }^{34}$

A joint study of the MoH and the WHO on cost-effective practices for NCDs and risk factors (using the 'OneHealth Tool') has shown that the total cost of NCDs amounted to TL 78 billion, corresponding to 3.3 percent of gross domestic product (GDP), in 2015.35

The burden of ambulatory care in hospitals is high in Turkey. The 2017 WHO STEPwise Approach to Adult Risk Factor Surveillance (STEPS) survey suggests that more than 81 percent of respondents' NCD care in hospitals was ambulatory (Figure 11) and shifting this to the primary care level is critical. The MoH statistics also support this fact as there is an increasing trend in hospital utilization since 2013, surpassing the FM utilization, with the exception of 2016-2017 (Figures 12 and 13).

Figure 11

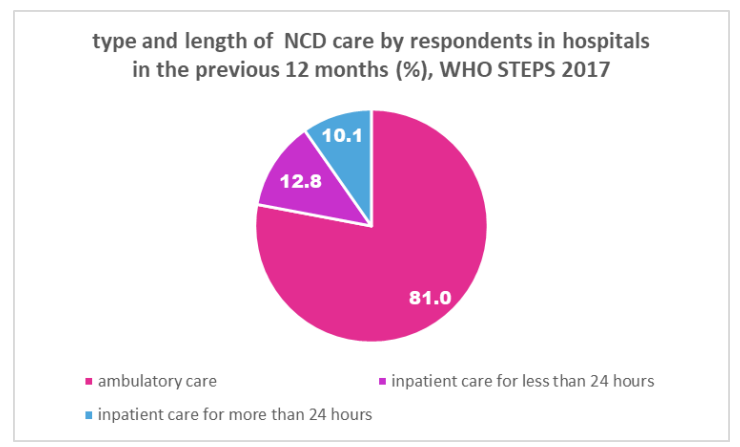

\section{Figure 12}

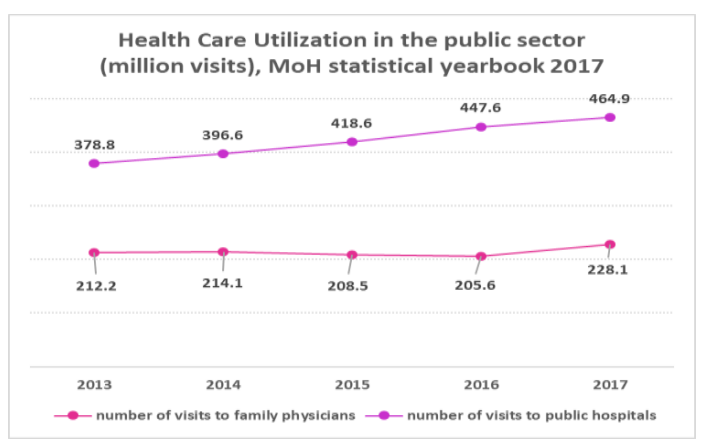

The MoH 2017 statistics also suggest that the hospital outpatient burden is extremely high and shows an increasing trend since the share of inpatient visits is less than 2 percent since 2013 (Figure 14). These data clearly show that the health care is mostly hospital centric and there is a need to shift ambulatory care to the primary care level to the extent possible.

\footnotetext{
33 WHO 2002.

34 Lyngsø et al. 2014.

$35 \mathrm{MoH} 2015$.
} 
Figure 13

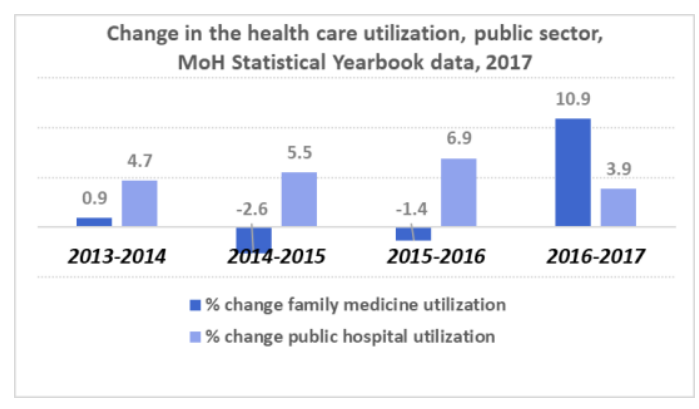

Figure 14

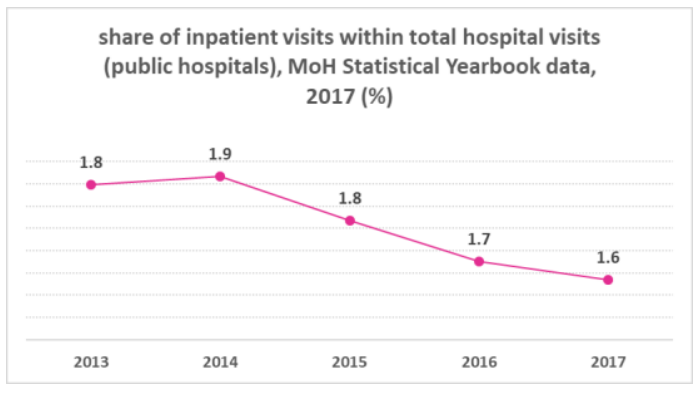

\subsection{INTEGRATED CARE AT HIGHER POLICY LEVEL IN TURKEY}

The MoH of Turkey, being aware of the pressures posed on the health system through the changing contextual factors, has shown commitment to address these challenges through its strategic plans. To this end, one of the goals of the Strategic Plan covering 2013-201736 is to provide accessible, appropriate, effective, and efficient health services to individuals and the community. The $\mathrm{MoH}$ intends to improve the integration and continuity of care by strengthening the role of PHC, especially through strengthening the integration of other PHC services into the FM model and through strengthening the integration of the FM model into hospital care and laboratory services. The plan also suggests strengthening the infrastructure and technological capacity of FM laboratory services, developing a system that will enable family physicians to consult with relevant specialists, improving the hospital appointment system by integrating it into the FM system, and improving laboratory services by integrating them into the FM system.

The Strategic Plan 2013-2017 also emphasizes the MoH's objective to control and reduce complications of NCDs through improving the quality of health care services for chronic diseases and establishing a system to manage the complications of chronic diseases by integrating PHC and hospital services and developing and using clinical quality standards.

The recently drafted MoH Strategic Plan 2019-2023 emphasizes the increasing burden of NCDs as their share in overall disease burden increased from 69.0 percent in 2002 to 82.4 percent in 2017. The plan highlights the need to improve health care delivery models and ensure continuity, proactive approaches, and close patient follow-up for NCD management.

One of the main goals of the new Strategic Plan focuses on preventing the exposure of the population to environmental risks and promoting healthy lifestyles through healthy nutrition and a physically active lifestyle and increasing health literacy. Another goal is to assign a central role to the FM system, to make patients responsible for their health (as stakeholders of health) and support the FM model through integrated care models which bring different disciplines ${ }^{37}$ together at the primary level. This new strengthened PHC model is expected to prioritize NCD management. The MoH intends to carry out systematic population-based screenings since early detection is one of the most important elements of NCD management. Both the screening processes and NCD management processes will be defined from end to end, standardized, and supported with technology. Physical infrastructure of PHC institutions will be improved and FMPs will play a more active role in the

36 https://sgb.saglik.gov.tr/Shared\%20Documents/OrtaAlan/15.02.2012\%20stratejik\%20plan.pdf.

37 Such as dietitian, physiotherapist, and psychologist. 
screening, diagnosis, patient education, and management of NCDs. Capabilities of PHC staff will be enhanced through improvements in the curriculum and in-house trainings.

Tables 1 and 2 provide the details of performance targets related to integrated care and NCDs and the integrated care related context of the MoH Strategic Plan 2019-2023.

Table 1

\begin{tabular}{|c|c|c|c|}
\hline $\begin{array}{l}\text { Indicators related to integrated care and } \\
\text { NCDs }\end{array}$ & 2019 value & $\begin{array}{l}\text { Planning period } \\
\text { baseline value }\end{array}$ & 2023 target \\
\hline Number of FMCs (cumulative) & 27,000 & $26,252(2018)$ & 32,190 \\
\hline Population per FMP & 3,069 & $3,098(2018)$ & 2,700 \\
\hline $\begin{array}{l}\text { Share of population who do not visit FMCs } \\
\text { within registered population }\end{array}$ & $22.26 \%$ & $22.40 \%(2018)$ & $18.25 \%$ \\
\hline Yearly per capita FMC visits (cumulative) & 3.3 & $3.1(2018)$ & 4.0 \\
\hline Number of HLCs (cumulative) & 205 & $193(2018)$ & 275 \\
\hline $\begin{array}{l}\text { NCDs whose screening and follow-up processes } \\
\text { defined at the primary level and integrated } \\
\text { within Disease Management Platform } \\
\text { (cumulative) }\end{array}$ & 3 & 0 (2018) & 7 \\
\hline $\begin{array}{l}\text { Colorectal cancer early diagnosis rate (phase } 0 \text { - } \\
\text { 1) }\end{array}$ & $35.5 \%$ & $35.4 \%$ & $37.0 \%$ \\
\hline Breast cancer early diagnosis rate (phase $0-1$ ) & $50.3 \%$ & $50.0 \%$ & $52.0 \%$ \\
\hline Cervical cancer early diagnosis rate (phase 0 ) & $58.0 \%$ & $57.8 \%$ & $59.5 \%$ \\
\hline HT screening coverage & $10 \%$ & $0 \%(2018)$ & $50 \%$ \\
\hline CVD risk assessment coverage & $20 \%$ & - & $60 \%$ \\
\hline Obesity prevalence (15+ population) & $31.0 \%$ & $31.5 \%(2017)$ & $29.1 \%$ \\
\hline Overweight prevalence $(15+$ population $)$ & $35.8 \%$ & $35.9 \%(2017)$ & $35.5 \%$ \\
\hline $\begin{array}{l}\text { Physically inactive population prevalence (15+ } \\
\text { population) }\end{array}$ & $38.0 \%$ & $39.6 \%(2018)$ & $32.0 \%$ \\
\hline DM prevalence & $12.4 \%$ & $12.5 \%(2017)$ & $12.1 \%$ \\
\hline HT prevalence & - & $27.9 \%(2017)$ & $24.9 \%$ \\
\hline Salt intake per capita per day & $9.4 \mathrm{~g}$ & $10.2 \mathrm{~g} \mathrm{(2017)}$ & $7.7 \mathrm{~g}$ \\
\hline $\begin{array}{l}\text { Level of awareness of activities concerning } \\
\text { health literacy }\end{array}$ & - & $30 \%(2018)$ & $60 \%$ \\
\hline $\begin{array}{l}\text { Share of population with sufficient or superior } \\
\text { health literacy }\end{array}$ & - & $31.1 \%(2017)$ & $40.0 \%$ \\
\hline
\end{tabular}




\begin{tabular}{|l|l|l|l|}
\hline $\begin{array}{l}\text { Indicators related to integrated care and } \\
\text { NCDs }\end{array}$ & $\mathbf{2 0 1 9}$ value & $\begin{array}{l}\text { Planning period } \\
\text { baseline value }\end{array}$ & $\mathbf{2 0 2 3}$ target \\
\hline $\begin{array}{l}\text { Number of nurses and midwives per 100,000 } \\
\text { population }\end{array}$ & 312 & $302(2018)$ & 350 \\
\hline $\begin{array}{l}\text { Share of population who are satisfied with FMC } \\
\text { services }\end{array}$ & $72 \%$ & $72 \%$ & $76 \%$ \\
\hline $\begin{array}{l}\text { Number of active profiles in e-pulse individual } \\
\text { health system platform }\end{array}$ & 11 million & 9.4 million (2018) & 15 million \\
\hline
\end{tabular}

\section{Table 2}

\section{Goal 1: Promote and expand healthy lifestyles}

Encourage healthy dietary habits through

(i) The introduction of incentive mechanisms that would encourage people for healthy dietary habits and physically active lifestyle;

(ii) Systematic communication activities to increase public awareness; and

(iii) Efforts to increase health literacy (increase awareness and competency of health workers on population health literacy, design digital platforms, applications, and processes that would facilitate increasing population health literacy and introducing health literacy volunteer model).

Goal 2: Strengthen PHC and increase its effectiveness within the health system

Provide multidisciplinary and highly preferred PHC through a strengthened FM structure.

Transform the primary care performance model so that it is quality based, satisfaction based, and results based for all stakeholders.

Establish standardized PHC processes where all steps are planned and incorporate related technological devices as well as ability to effectively follow patients in the processes.

Improve primary care staff capacity and qualifications through improvements in specialization programs and training curricula and through in-house training.

Goal 3: Ensure accessible, effective, efficient, and high-quality care delivery

Improve clinical quality of health care.

Ensure effectiveness and productivity for outpatient and emergency care.

Improve accessibility and sustainability of health care services.

Goal 4: Introduce Integrated Health Care Model

Carry out NCD screening at PHC institutions on HT, DM, and CVD risk assessment.

Establish a regular individual-based NCD follow-up system which would incorporate information on different areas such as patient history, patient's other medical conditions, patient's communication preferences, and behavioral patterns.

Change the public perception toward PHC by improving the PHC strategic design to include the following elements:

(i) Design a new PHC service delivery model where PHC will assume a priority role for NCD follow-up. Ensure that the FM system is at the center and patients are the continuous health stakeholders. To support and strengthen the FM model, introduce different disciplines such as dietitian, physiotherapist, and psychologist to operate at the PHC and provide integrated services.

(ii) Redesign the FM performance system to be more effective and to include elements such as quality, satisfaction, and resultsbased focus and to reflect the MoH priorities.

(iii) Design all PHC processes end to end for long-terms cases such as NCDs. The processes should be standard and should aim to improve the patient experience. The processes will include case-based clinical treatment protocols, decision trees, service points, actors to assume the roles, and service delivery flow diagrams. Clinical and administrative processes also have to be thoroughly reflected to the HIS and there should be perfect information flow between stakeholders. 


Increase PHC staff competencies through effective FM training schemes that are redesigned and improved to reflect effective
NCD management at the primary level.
Increase effectiveness of the early diagnosis and management of complications of NCDs.
Prevent, maintain, and improve the mental health of people.
Strengthen the multistakeholder governance model to improve health responsibility.
Goal 5: Ensure patient and provider satisfaction and health system sustainability
Increase patient satisfaction.
Continuously improve health worker satisfaction.
Increase the use of information technologies in the health care provision and decision-making processes to strengthen the health
system.
Increase the effectiveness of human resource planning and skills building.
Improve effectiveness and efficiency of health management.

\section{ESSENTIAL COMPONENTS OF INTEGRATED CARE: OVERVIEW, COUNTRY EXAMPLES,} STATUS OF TURKEY, AND RECOMMENDATIONS

The widespread move toward integrated care is being driven by the increasing burden of chronic disease, multimorbidity, and high-risk populations, which present an overwhelming case to better manage the processes and outcomes related to the needs of the population that a health care system serves. Evidence suggests that an integrated care approach can improve care quality, patient/staff experience, process efficiency and effectiveness; reduce costs; and improve patient outcomes and well-being. ${ }^{38}$

Integrated care occurs on different dimensional areas of the health system, including organizational, functional, and service integration. Organizational integration usually includes formal mergers of health care provider systems (bringing together different sectors of the health care) or establishing collective structures for coordinated provider networks often through contracts or policy. Functional integration recognizes and necessitates the need to integrate nonclinical business practices (such as back-office activities) and electronic patient records. Service integration focuses on bringing different clinical services together at the organizational level through teams of multidisciplinary professionals. ${ }^{39}$ (Figure 15)

\section{Figure 15}

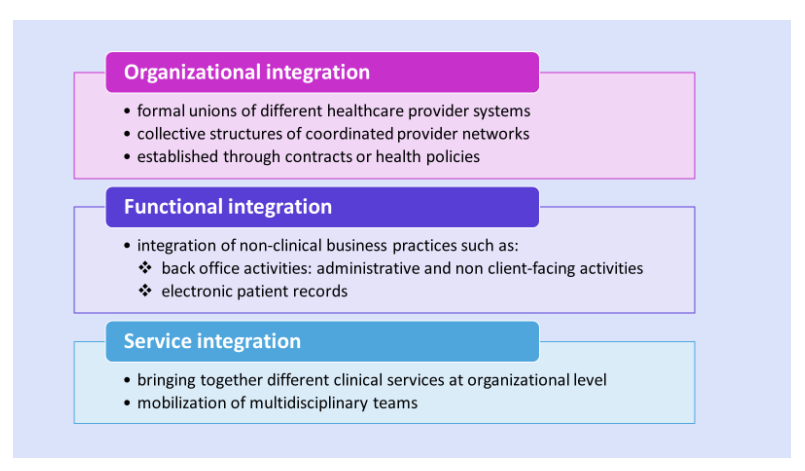

\footnotetext{
38 Bertram et al. 2018; Rosland et al. 2017.

39 WHO 2016b.
}

\section{https://www.nuffieldtrust.org.uk/files/2017-01/where-next-integrated-care-english-nhs-web-final.pdf.}


Integration is often referred to as vertical or horizontal. Horizontal integration is defined as establishing formal care delivery between two or more health care entities at the same stage/level in the process of care, such as integrating two hospitals. Vertical integration involves organizations addressing care needs at different levels of care such as primary care and secondary care or general practice and the community health care. In terms of the scale, integrated care can be implemented at three levels: micro, meso, and macro. The micro level focuses on the individual care experience made through a personalized care plan. The meso level represents integrated care for a specific group or population with the same disease or condition. Implementing care at the macro level entails needs stratification for an entire population and then tailoring services to attend to those needs.

Depending on the level of intensity, integrated care models range from partial to complete. While the latter aims to integrate health and social sectors into a new organizational model, the former is about forming voluntary links to support integration and to enhance coordination between health and social sectors. ${ }^{40}$ Some integration models focus on the needs of high-risk groups, others focus on groups with specific chronic diseases. $^{41}$

The WHO outlines three models of integrated care: (a) individual models where the goal is to facilitate coordination among providers to reduce fragmentation and to improve chronic illness/disease management (case management, individual care plans, patient-centered medical home, personal health budget); (b) models that target wider groups of patients (chronic care model, integrated models for frail and the elderly, and disease-specific models); and (c) population-based models (Kaiser Permanente USA, Veterans Health Administration, USA [VHA], and Integrated Care Basque Country). ${ }^{42}$ The WHO notes, however, that the broader models often contain subsets and portions of disease and case management aspects found in individual models of integrated care.

As the integrated care approach aims to improve access, quality, and continuity of health care services, the role of the primary care in integrated care settings becomes crucial. In fact, the basic functions of the primary carefirst point of contact; continuous, comprehensive, and coordinated care-make primary care the starting point for efforts to improve and integrate care. The integration of the biomedical, psychological, and social dimensions of health and well-being is the core value of primary care and addresses both the concepts of person-focused and population-based care, which constitute the guiding principles to achieve better care coordination across the care continuum. Health systems where the principles of primary care (first point of contact; continuous, comprehensive, and coordinated care) actively function achieve better health outcomes and improved health care equity when compared with systems focusing on specialty care. In fact, several highincome country settings reveal that health care service integration is disrupted by the existence of fragmented health service provision which in turn brings about suboptimal care and increased costs due to duplication and poor quality of care. 43

There is now substantial evidence that outlines the essential components of integrated care, delivery strategies, success factors, and challenges faced by many countries and health care systems as they move to transform their health system toward a more integrated model.

\footnotetext{
40 WHO 2016b.

41 WHO 2016.

42 WHO 2016.

43 Valentijn et al. 2013.
} 
Even though the definition of integrated care is varied and often viewed and expressed within different contexts, key components of integrated care are emerging from the literature. ${ }^{44}$ These include engaged leadership, a focus on patient centeredness, engagement and self-management support, development of a multi-professional team and work culture, adoption of evidence-based clinical pathways and protocols, care coordination and case management, alignment of incentives for participation, effective management of resources, continuous monitoring and performance improvement, and investment in supporting technologies. These components are the foundation for practical approaches that guide organizations to successfully implement integrated care practices. This report identifies four major dimensional areas that describe the key components of integrated care found in the literature: (a) fundamentals of change, (b) organizational change, (c) care delivery, and (d) the use of technologies. The sections below further detail the critical elements of integrated care under these dimensional areas and also present good country practices for each of these elements. Real-life approaches to achieve improved and integrated care delivery and improved capacity within the PHC system include lessons for leaders, stakeholders, and staff alike. "Well performing health systems require attention to design, implementation and monitoring." 45 Therefore, the following sections outline specific strategies and actions taken in the identified countries as they strive for an integrated care system. Country examples are selected to be relevant and guiding for the Turkish context, and they aim to provide insight to Turkish policy makers for the integrated care efforts.

\subsection{FUNDAMENTALS OF CHANGE}

\subsubsection{ENGAGED LEADERSHIP AND SHARED VISION}

3.1.1.1 OVERVIEW AND COUNTRY EXAMPLES

Published literature identifies the key role of national, regional, and local leadership in facilitating change transformation. Leaders are responsible for charting the course for change and supporting and ultimately sustaining all associated efforts for transformation. One of the key roles of leaders during transformation is to identify and allocate resources (staffing, time, equipment, technology/tools, education funding, and so on) to implement and maintain efforts. Engaged leaders are visible and active in working with staff to identify issues and support solutions. Engaged leaders provide direction, motivation, and resources through strategies such as making the case for transformation and communicating extensively and effectively, identifying and mentoring champions, using data to drive and guide improvement, and ensuring adequate resources for the work. ${ }^{46}$ The literature on leadership, clinical governance, and/or advisory groups is well studied. Several authors recommend specific approaches for leadership, governance, and/or advisory committee engagement to effect large system change and improvement. ${ }^{47}$

Strong leadership and shared vision at all levels are critical to successful change within a health care system, including formal and informal leaders at each level. Leadership is important for articulating a shared vision and conveying the urgency and importance of that vision to all stakeholders. Establishing a strong policy framework

\footnotetext{
44 Evans et al. 2016.

45 Cotlear et al. 2015.

46 Reinertsen, Bisognano, and Pugh 2008; Safety Net Medical Home Initiative 2013a.

47 Barker, Reid, and Schall 2016; Best et al. 2012; Nolan et al. 2005; Reinertsen, Gosfield, and Rupp 2007; Willis et al. 2016
} 
to support reforms, define execution, and communicate all through various media is important. Leaders also secure and dedicate needed resources to enable transformational changes. They provide a supportive organizational culture by promoting open and honest feedback from key stakeholders, providing for transparent monitoring and evaluation and shared decision making to foster public trust. ${ }^{48}$ Appointing an executive-level leader with subject matter expertise to spearhead the implementation effort provides more focus on the effort and has demonstrated success in other countries by addressing fragmentation, miscommunication, and conflict of interests. ${ }^{49}$

Scotland and Japan are two good examples of strong and engaged leadership.

The case of Scotland is a good example for demonstrating a committed leadership where plans are put in action. Facing similar pressures of many countries on addressing changing demographics and the health problems of ageing populations, the Scottish government, Confederation of Scottish Local Authorities (COSLA), and National Health Service (NHS) established in 2009 a program 'Reshaping Care for Older People' to improve care and make health services more sustainable. In 2010, the report of the Christie Commission on the Future of Public Services followed this effort and suggested an approach for health care service integration which prioritizes person-centered care, partnerships for seamless care, and prevention and close monitoring of health care services. These themes constituted the Scottish government's 2020 vision and plans to integrate health and social care across Scotland. After the May 2011 elections, the Scottish government had the support of all political parties and was able to enact integrated care. The Public Bodies Act of 2014 was the primary legislation for integrated care, mainly focusing on personal and population health outcomes rather than inputs. In December 2011, the Scottish government's plan to integrate adult health and social care was announced. The Public Bodies Bill presenting the legislative framework for integrating health and social care was introduced in the Scottish Parliament in May 2013. The bill emphasized the need to improve quality and consistency of health care and to provide seamless health and social care especially for people with longer-term and complex health needs. To remove barriers against joint working between NHS boards and local authorities, new public organizations, known as integration authorities, have been established by the Public Bodies Act of 2014. In addition to the requirement of integrating health and social care budgets for NHS boards and local authorities, the Public Bodies Act necessitated the introduction of nationally agreed outcomes and partnerships which will strengthen the role of clinicians and health care professionals in the process of health care planning and delivery. 50

The Japanese Mitsugi prefecture case is a good example to show the commitment and genuine engagement of stakeholders for integrated care at the local level. The head of the Mitsugi prefectural hospital, members of the prefectural government and town council, and the town residents and health professionals were the stakeholders for the community-based integrated care initiative in Mitsugi, which started in the early 1970s. As a local leader, the Hiroshima prefectural governor was highly interested in Yamaguchi's plan to serve the elderly within the community as he was struggling to meet the increasing demand for elderly care. In line with this interest, the local authorities supported Yamaguchi's plan and approved the integration of medical, preventive, and welfare services. This move made the use of the health workforce in a flexible manner. To

\footnotetext{
48 WHO 2015.

49 WHO 2015.

50 Bruce and Perry 2015.

https://akademia.nfz.gov.pl/wp-content/uploads/2016/12/Raport Opieka_koordynowana_ENG.pdf. http://www.parliament.scot/ResearchBriefingsAndFactsheets/S5/SB_16-70_Integration_of_Health_and_Social_Care.pdf
} 
illustrate, community/public health nurses in Mitsugi who were originally employed by the municipal government were able to be employed by the hospital with the changes toward integration. ${ }^{51}$

On a broader leadership engagement level, as an attempt to respond to the rapid ageing of the Japanese population, an integrated community care system was introduced by the Japanese government in 2006 to integrate preventive, medical, and long-term care as well as to provide living support and social care. The new care model is managed by municipal governments and is financed under a long-term care insurance fund. It aims to create an environment for the elderly where they can live with dignity with sufficient social support. Integrated community care model presents an important change in the Japanese health system as reflected in the high-level policy document 'Japan Vision: Health Care 2035'52 that suggests a shift from inputs to outcomes, from quantity to quality, from cure to care and prevention, and from specialist practices to an integrated perspective. Concerning integrated care, Japan Vision: Health Care 2035 suggests the introduction of concepts such as the implementation of value-based care, empowerment of society, and encouragement of personal choice for life design. ${ }^{53}$

Ensuring a high-quality health care workforce is another requirement for leadership to apply when driving system transformation and promoting capacity to deliver services. "Governments provide the political leadership, resolve and resources to effectively steward the education, deployment, management, financing and performance of a health workforce that equitably serves population needs, promotes the right to health and accelerates progress towards population-specific, comprehensive universal coverage". ${ }^{54}$ The WHO emphasizes that countries are responsible for ensuring a high-quality health care workforce by developing a national strategy to address gaps in numbers, distribution, and retention; modernizing training curricula and integrating principles of quality and improvement methods; and encouraging programs of professional development and evaluating their impact. 55

Improvements in developing a high-quality health care workforce in Brazil, Ghana, Mexico, and Thailand reveals the following:

(a) Training more staff is necessary but trainee selection, location, content and mode of training, and development of appropriate skills for an effective mix across multidisciplinary teams is essential.

(b) Employing more staff in underserved areas is necessary but not sufficient to improve quality and access. Appropriate geographic distribution which is complemented with the right bundle of financial and nonfinancial incentives is required to recruit and retain. Therefore, fully funded human resource policies are critical.

(c) Addressing deep-seated health system bottlenecks requires sustained investments, recurrent cost budgets for staffing, education and incentives, and a policy focus over a longer period. Adequate policies and funding are crucial to achieve a skilled and motivated workforce as well as supplies, equipment, infrastructure, and financing to provide quality care..$^{56}$

\footnotetext{
51 Hatano et al. 2017.

52 Miyata et al. 2015; Reich and Shibuya 2015.

53 Japan health system review http://apps.searo.who.int/PDS DOCS/B5390.pdf.

54 OECD 2016.

55 WHO $2016 \mathrm{c}$.

56 Campbell et al. 2013.
} 
Turkey has demonstrated a successful and important example of leadership engagement in the last decade to improve and take the Turkish health system forward. Drawing on several factors (including demographic and epidemiologic changes, health system infrastructure, health human resources, and care delivery mechanisms on different levels of care) necessitating reforms, Turkey's HTP had the leadership support and commitment and started in 2003. The HTP took citizens as the main focus and brought about remarkable changes to the Turkish health system. A more-balanced distribution of health human resources throughout the country contributed to increased access to care and population coverage and improved equity. The program was successful in introducing the FM model in $2005^{57}$ with the underlying idea to shift care from hospitals to PHC by increasing physical access to care and by addressing PHC issues at the FM level rather than at hospitals as a less costly mechanism. Currently, while the $\mathrm{MoH}$ has a number of efforts to strengthen the primary care, the presence of fragmented policies and structures constitutes an obstacle for more effective approaches toward integrated care.

At the ministerial level, interviews with department leaders at the local, regional, and national levels of government reveal a lack of common vision, understanding, and consensus around the implementation of the MoH Strategic Plans, resulting in local and regional systems to define, develop, and implement their unique version. This might result in a lack of standardization which can negatively affect national goals. Without a common vision and shared understanding-including well-defined approaches-national, regional, and local leaders cannot fully subscribe and implement as the $\mathrm{MoH}$ had intended.

Health human resources is another important area to be addressed in Turkey through active and effective engagement of leaders. As has been indicated in the MoH Strategic Plan 2019-2023, the health workforce does not have enough staff to address the demand for health care and for institutional needs. The number of FMPs could not be increased as planned. The international comparative data 58 also suggest that Turkey's performance is below the European Union (EU) averages in terms of the number of general practitioners and nurses (Figures 16 and 17). Therefore, managing the capacity building, deployment, and performance improvement of health workers is critical to ensure quality and equity of care. ${ }^{59}$

Figure 16

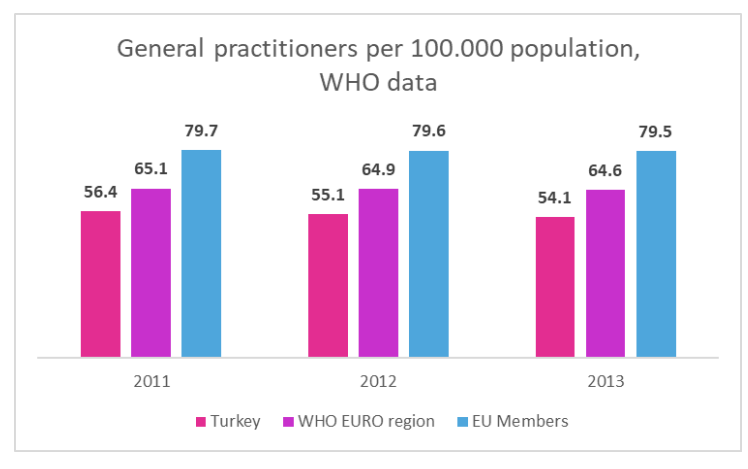

Figure 17

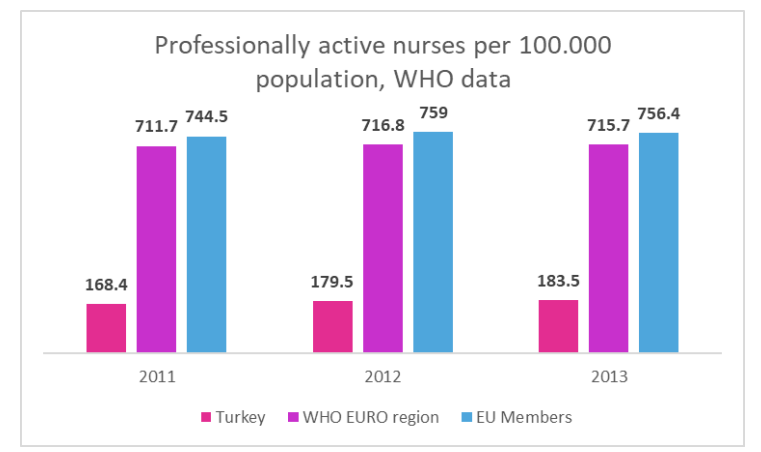

\footnotetext{
${ }^{57}$ Family medicine system was rolled out throughout Turkey in phases (started in 1 pilot province in 2005, 6 provinces joined in 2006, 7 provinces in 2007, 17 provinces in 2008, 4 provinces in 2009, and finally 46 provinces in 2010).

$58 \mathrm{https://gateway.euro.who.int/en/indicators/hlthres} \mathrm{71-general-practitioners-per-100-000/.}$

59 The $\mathrm{MoH}$ indicates the number of physicians per 100,000 population as 188 for 2018 and 230 as 2023 target. The number of nurses and midwives per 100,000 population is indicated as 302 for 2018 and 350 as 2023 target.
} 
Additionally, the recent and frequent organizational restructurings and changes in the MoH administration had negative effects on the MoH's management and operations. These changes prevent the MoH administration from establishing an open and functioning communication channel with the health care providers (in public and private sector).

\subsubsection{RECOMMENDATIONS ON INTEGRATION WITHIN PRIMARY CARE LEVEL}

The recommendations below concentrate on ensuring a shared vision, common understanding, and consensus at various leadership levels which will help reduce fragmentation.

Have a champion for integration. The MoH Strategic Plan identifies a number of different MoH program executives and directorates as accountable leaders for integrated care which makes a unified approach to integrated care execution difficult. Therefore, having a political or technical champion to lead this important transformation effort may provide more focus on the effort and address fragmentation, miscommunication, and conflict of interests. The champion would be responsible and accountable for ensuring that all MoH central and provincial units are formally and actively engaged in joint planning, strategizing, and fulfilling the $\mathrm{MoH}$ goals for integrated care. The setting would also hold all engaged parties equally accountable for the progress toward integrated care.

Develop a complementary detailed integrated care strategy/policy. The MoH Strategic Plan and guidance have provided useful information; however, interviews and discussion with local HLC's and provincial leaders emphasize a need for more specific and standardized implementation guidance and/or policy to ensure a standardized approach to regional and local integration as envisioned. The champion for integration, as recommended earlier, would be the natural and most appropriate person to be tasked with development and publication of more specific and targeted materials, tools, guidance, and policy. ${ }^{60}$ This will ensure a more standardized approach to implementation, monitoring, and performance, fostering quick wins for short- and long-term goal achievements.

\subsubsection{PATIENT FOCUS AND ENGAGEMENT}

\subsubsection{OVERVIEW AND COUNTRY EXAMPLES}

The global literature consistently demonstrates that patient centeredness, person focused, patient engagement, and activation are foundational elements found in all models of integrated care. ${ }^{61}$ Research also links this key element to positive health outcomes, improved relationships and communications with the health care team and patient satisfaction, better recall of information and treatment adherence, better recovery, and improved health outcomes. ${ }^{62}$ "Patient-centered care is considered to be care that is relationship-based and makes the patient feel known, respected, involved, engaged, and knowledgeable."63 Engaging patients in their health care and self-management is essential in addressing their needs and managing both medical and social determinants of health, which are the primary influencers and often drivers of health for individuals. ${ }^{64}$

\footnotetext{
${ }^{60}$ A more detailed discussion on these issues can be found in Sections 3.2.2.3, 3.2.4.3, and 3.3.1.3.

61 Oni et al. 2014; Struckman et al. 2017; WHO 2013.

62 Schottenfeld et al. 2016.

63 Schottenfeld et al. 2016.

64 Gulliford 2017; Shi et al. 2002.
} 
For people with multimorbidity, there is a need for guidelines that prioritize recommendations for individuals and also compare relative benefits or risks. Population risk stratification based on care needs is a necessity to allocate resources and to plan personalized and patient-centered care delivery. ${ }^{65}$ The emerging concepts of self-care, co-ownership of care delivery options, discussion on planned outcomes, and informed expression of choice call for revisiting professional identity of health care workers. ${ }^{66}$

For a health care system to shape services as people-centered, robust feedback from the population is required. Approaches often include general patient surveys, often mailed, but they do not provide specific feedback regarding the who, what, when, and where. Therefore, health care systems aspiring to transform health care provision toward a people-centered perspective and to use patient experience to better meet the needs of the population are moving toward additional approaches that provide patient feedback with methods that are more regular and in real time, along with routine surveys. ${ }^{67}$ These approaches include establishing patient advisory boards at the local (for example, FMC/HLC), regional, and national level delivery systems; implementing point-of-care-specific brief surveys following a clinic visit; conducting focus groups at a clinic to elicit feedback on a specific topic of service delivery or operation; and implementing secret shopper programs whereby a person interacts with the clinic as an undercover patient to observe workflow and operations and provides feedback about his/her experience to clinic management. All these methods provide a much more usable and targeted approach to improvements in service delivery and are part of a comprehensive program of people-centered health care.

The cases of Germany and Japan are noteworthy for patient focus and engagement.

Germany's Gesundes Kinzigtal scheme is a good example that promotes active patient engagement. The program's founding actors strongly believe that improving patients' self-management capabilities and encouraging their active participation in treatment decisions are important to ensure sustained gains in overall population health as well as to transform patients to become the 'co-producers' of their health. 68

The Gesundes Kinzigtal scheme pursues a shared decision-making process for health care provision. It includes joint development of individual treatment plans as well as treatment goal-setting agreements between the physicians and enrolled patients. Jointly developed treatment plans and goals to be achieved are continuously evaluated and patients are offered with additional educational programs. ${ }^{69}$ The model provides support to physicians in terms of know-how for case management and financial incentives and encourages patients' active participation in the process by offering them services such as patient education programs, self-help initiatives, and support in health-related decision making. ${ }^{70}$ On a broader institutional level, a structure comprising a

\footnotetext{
65 Onder etal. 2015.

66 Borgermans and Devroey 2017.

${ }^{67}$ Department of Health Australia 2015; Hempel 2018; Safety Net Medical Home Initiative 2013b, 2014.

68 https://www.ncbi.nlm.nih.gov/pmc/articles/PMC2914874/pdf/ijic2010-2010046.pdf.

69 The Gesundes Kinzigtal program, Germany. http://www.icare4eu.org/pdf/Gesundes Kinzigtal.pdf.

70 One example of patient support schemes is the active health promotion for the elderly program which is a half-day patient education program focusing on health literacy for nutrition, physical exercise, and social participation and aims to promote and sustain healthy behaviors on the part of the patients. The half-day course is then followed by continuing support through regular letters and refresher workshops.
}

Another example for patient-focused support is the healthy body weight intervention program for patients with high blood sugar level, high body mass index, and lipid metabolic disorder and involves interventions concerning nutrition and physical exercise with regular checks of blood sugar level, performed either by physicians or by patients themselves. 
patient ombudsman and a patient advisory board is established to incorporate patients' perspectives to the managerial decision-making processes. The patient advisory board has an intermediary role between patients and the health care service partners and ensures that the patients are involved in the development of the

Self-management training program involves both coaches and patients with multimorbidity to improve patients' coping skills and better organize patients' daily activities and family lives, irrespective of their specific diseases

http://www.icare4eu.org/pdf/Gesundes Kinzigtal.pdf 
programs. The ombudsman has the role to solve/address the problems by meeting with the people concerned. ${ }^{71}$

The Mitsugi community-based integrated care model in Japan is another good example. The success of this local initiative with extensive focus on patients later made Mitsugi system a model for nationwide integrated care. ${ }^{72}$ In fact, one of the main drivers to introduce a new model was the high number of old people and the significantly higher share of bedridden elderly population in the town. ${ }^{73}$ Many people who had a stroke and got acute treatment in a hospital became bedridden due to inadequate post-acute care at home and separate provision of hospital outpatient and inpatient services, and the long-term care made the service provision difficult. The concerns of Mitsugi municipal government and a number of health professionals in the town led them to take action. Home-based and integrated care services were therefore introduced to address the needs of bedridden patients and their families.

\subsubsection{CURRENT STATE IN TURKEY}

The HTP has introduced a number of mechanisms concerning patient focus and engagement. These include the patient's right to choose physicians, the establishment of patient feedback systems (such as the patient right units in hospitals and a hotline called SABIM [MoH Communication Centre] to collect feedback on patient complaints and suggestions and regular patient satisfaction surveys). In addition to these efforts, the MoH also aims to improve population health literacy as indicated in its Strategic Plans. ${ }^{74}$

Turkey's health literacy rates are considered low when compared to the EU (Table 3). The awareness of population on risk factors for chronic diseases is also low, particularly for dietary and physical exercise habits (Figure 18). Low health literacy is a primary contributing factor in health disparities and patients with low health literacy understand less about their medical conditions and treatments and overall report worse health status. Therefore, improving health literacy for Turkey is paramount for effective and continuous patient engagement.

\section{Table 3}

\begin{tabular}{|l|l|l|l|l|}
\hline $\begin{array}{l}\text { Health literacy in } \\
\text { the EU and Turkey }\end{array}$ & Inadequate (\%) & Problematic (\%) & Sufficient (\%) & Excellent (\%) \\
\hline EU75 $^{70.4}$ & 35.2 & 36 & 16.5 \\
\hline Turkey $^{76}$ & 24.5 & 40.1 & 27.8 & 7.6 \\
\hline
\end{tabular}

71 https://www.ncbi.nlm.nih.gov/pmc/articles/PMC2914874/pdf/ijic2010-2010046.pdf

Healthy Kinzigtal Germany http://chrodis.eu/wp-content/uploads/2017/03/healthy-kinzigtal.pdf.

72 The rollout of the program in other districts started in 2005 where the Long-term Care Insurance Act has been also revised. The centers include teams of public health nurses, social workers, and care managers and are responsible for preventative care services, outreach, and counselling for elderly patients, and continuous and comprehensive care management support.

${ }^{73}$ Elderly people accounted for 16.7 percent of the population, exceeding the national average of 9.1 percent. Additionally, the number of bedridden populations living at home in 1980 was 30 percent higher in Mitsugi than in all of Hiroshima prefecture.

75 https://www.ncbi.nlm.nih.gov/pmc/articles/PMC4668324/pdf/ckv043.pdf.

76 http://www.sagliksen.org.tr/cdn/uploads/gallery/pdf/8dcec50aa18c21cdaf86a2b33001a409.pdf 


\section{Figure 18}

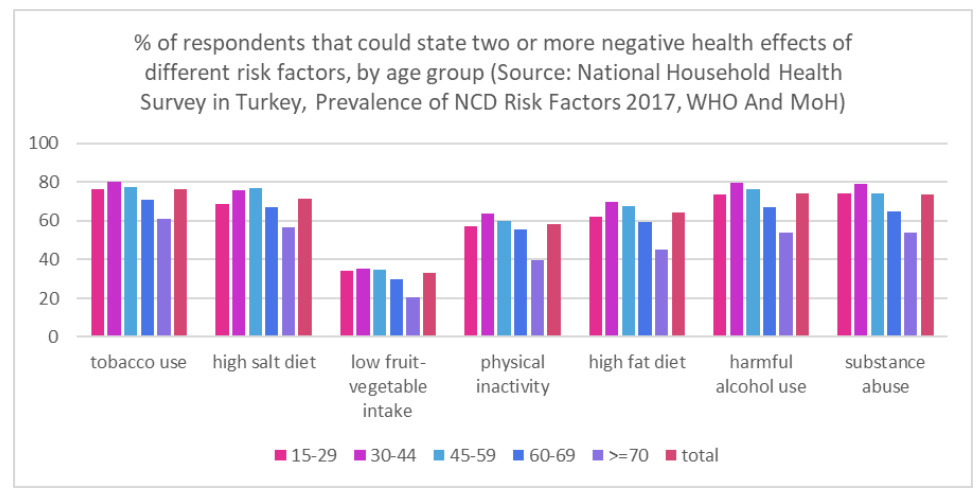

The MoH Strategic Plan 2019-2023 77 indicates the aim to develop a patient-centered care system and to promote patient engagement. To promote patient engagement, the Strategic Plan indicates the MoH's intention to encourage and improve healthy dietary habits and physical activity efforts, to carry out systematic communication activities to increase public awareness on healthy lifestyles, and therefore to transform the population to have high health literacy and high awareness on self-health, and use the health system effectively. The Strategic Plan also highlights the introduction of integrated care models that require cooperation of different levels of care, and one of the strategies related with this goal is to increase patient participation in the management of disease prevention.

While the new Strategic Plan shows the MoH's intention to improve and expand efforts on patient engagement, how this will be done still remains unclear, as concrete action plans to ensure full patient engagement and patient self-management at the primary care level, which constitute an important pillar of patient centric care, have not been presented. There are, however, local initiatives such as the one in Bursa Osmangazi.

Interviewees from Bursa and Hatay HLCs highlight that low health literacy among population is an issue. To promote awareness and increase health literacy, regular public training sessions are held in Bursa every Thursday. Topics for the public trainings are selected among those areas where patients mostly seek advice and solutions. The training content for each of these topics is then standardized and adjusted to increase population coverage for training. Currently, the public training content focuses on cancer, NCD risk factors and prevention, coping with stress, ergonomics, back pain, and healthy eating habits. Bursa HLC staff also work face to face with patients in the field to increase patient-provider dialogue. Hatay HLC staff have worked with around 530 volunteered young people on a number of health issues including obesity control, health literacy, and rational drug use to create population awareness.

HLCs also make efforts to provide individual care to their patients. The qualitative fieldwork carried out in July 2018 within the scope of the PHRD grant (a project implemented jointly with the World Bank and the General Directorate of Public Health) included interviews with patients, and these interviews reveal that patients are satisfied with HLC services as they report that there is one-to-one counseling and customized service/care in HLCs. Interviewed patients also indicated that HLC specialists understand them and they are cordial and communicate to them in a language/tone they can understand. Patients highlighted that specialists give answers that convince them.

76 http://www.sagliksen.org.tr/cdn/uploads/gallery/pdf/8dcec50aa18c21cdaf86a2b33001a409.pdf

77 This recent plan is currently not published. 
Another good example that would promote patient engagement is an effort under the PHRD grant which aims to increase the appeal of primary care utilization through the nudges (small, non-mandatory interventions that are expected to induce changes in people's behavior). The results and lessons learned from this project are yet to be seen and they might provide insight to design broader efforts for patient engagement.

\subsubsection{RECOMMENDATIONS ON INTEGRATION WITHIN PRIMARY CARE LEVEL}

Develop and disseminate a nationwide comprehensive health literacy program. Improving health literacy is an important part of the MoH Strategic Plan that notes a need to develop capacity-building programs for trainers and teachers of health literacy and to strengthen communication efforts aimed at improving the health literacy of the populace.

Health literacy represents a significant barrier and challenge to improve the health and well-being of the patients treated in HLCs and FMCs. Topics to be included in a comprehensive health literacy program should address NCDs and prevention care and should be available in printed form, online, through social media, and in e-pulse at a low-grade reading level with clear illustrations and/or with audio means, as appropriate.

Develop and implement a nationwide program to improve patient engagement. As the Gesundes Kinzigtal case of Germany highlights, patient engagement in terms of self-management, active participation in treatment decisions and plans, and shared decision making are key in integrated care. Therefore, consistent with the MoH Strategic Plan, a targeted approach to the topics that support patient engagement, patientcentered care, and self-management is recommended to be developed and disseminated widely. Specific approaches and topics known to be successful in other countries include (but are not limited to) the following:

1. Required training of all FMC and HLC staff on communication techniques with patients (teach back, motivational interviewing, $5 \mathrm{As}^{78}$, and so on). This will improve the effectiveness of the patient-provider dialogue.

2. Development of self-management support tools, sometimes called as a health library, ${ }^{79}$ for patients and staff to use and make available across all types of informational media. The $\mathrm{MoH}$ might consider introducing the option of a health library through e-pulse as this is a strong platform.

3. Survey of patients regarding their needs and experiences at the point of care. This can be done through small printed cards that anonymously ask about the patient's immediate opinion/impression on that specific visit, before he/she leaves the FMC or HLC. Aggregated results of these mini surveys should be available to FMC/HLC staff and be used to improve processes and care delivery.

4. Provision of self-management support at every visit with professional staff. This practice requires the health workers to regularly ask generic questions to the patient on how he/she cares about him/herself, reiterating the tasks the patient is supposed to do himself/herself.

5. Provision of proactive care management and outreach to patients with NCDs and prevention care needs. This involves actively contacting patients and encouraging them to either visit their care providers or to counsel them virtually (like over the phone) for care without waiting for them to seek care by themselves. To

\footnotetext{
78 The 5As (Ask, Advise, Agree, Assist, Arrange) are a clinical tool recommended for health behavior counseling in primary care (https://www.ncbi.nlm.nih.gov/pubmed/23110376).

79 The library includes information and guidance to patients. One good example is the diabetic feet examination guidance and information for diabetic patients.
} 
illustrate, these counseling sessions or visits can include dialogues with patients to check whether they follow their medication regimen, follow their care scheme, do the regular checks, and so on.

6. Improvement of the personal health record interface of the e-pulse app platform through the introduction of new tools that can be used directly by the patients. This will increase the patient engagement through improved perception on self-management and encourage the patients to more effectively enter their personal health information (such as personal health plans and self-management goals) that can be used to facilitate a partnership between the FMC/HLC health team and the patient. Through this partnership with the patient, the FMC/HLC health team can follow up on the progress of the patient and can give feedback.

These actions will facilitate the development of a culture for patients to be more active and eager to take part in their health care decisions and practices.

Establish patient advisory boards. Similar to the Gesundes Kinzigtal case, where patient ombudsman and patient advisory board structures are in place to consider the patient perspective in the managerial decision making, Turkey can also form such structures or can improve its patient feedback mechanisms. Both approaches are important because they aim to obtain feedback from patients and patients' families about their health care experience, to be used for quality improvement.

The MoH can establish patient advisory boards or include patient representatives on existing boards at the local, regional, and national levels. Concerning the patient feedback, the MoH Strategic Plan already substantiates the importance of receiving feedback from the Turkish populace regarding the health care system and discusses continuing to conduct surveys for patient satisfaction, include patient surveys in the Monitoring and Evaluation Framework, and set targets for improvement. ${ }^{80}$ These efforts also need to be complemented with more real-time methods such as immediate point of care mini patient surveys, patient focus groups, and secret shopper programs. ${ }^{81}$ As discussed in the overview section, these new approaches of eliciting feedback from patients are more pertinent to their experiences in a clinic visit or in their FMC.

\subsection{ORGANIZATIONAL CHANGE}

The provision of integrated care and redesign of health care services imply changes in the health care structures, organization of workflows, workforce development, and resource allocation to provide more responsive care delivery. There are a number of ways which facilitate the process of integrating health and social care programs and reorganizing systems, services, and care processes. ${ }^{82}$ As elaborated in the following sections, critical elements proven to drive organizational change include addressing regulatory frameworks, creating an enabling environment to foster change and implementation, aligning finances and incentives that support integrated care delivery, and establishing a robust monitoring and performance improvement system.

\footnotetext{
80 The Strategic Plan 2019-2023 indicates that the health reforms helped increase patient satisfaction but also contributed to raise patient expectations from health care delivery. The plan highlights that increased expectations are among the factors for recently stagnating patient satisfaction figures. To consider patient expectations in more detail and more effectively, the MoH intends to introduce new measurement methods which will be coherent with the health system and which will continuously and systematically keep track of patient satisfaction. This will help the MoH improve the health system and the care delivery based on changing patient expectations.

${ }^{81}$ A secret shopper is defined as someone employed to test the service in shops and businesses by pretending to be a normal customer.

82 European Union 2017.
} 


\subsubsection{REGULATORY FRAMEWORKS}

\subsubsection{OVERVIEW AND COUNTRY EXAMPLES}

The 2018 WHO publication Global Coordination Mechanism on NCDs Working Group on the Inclusion of NCDs in other Programmatic Areas report emphasizes "the need for governments to ensure adequate resource allocation to NCD care in primary health care with political commitments, legislation and regulation, and coordinated care. The report also stresses that "to strengthen the integration and management of NCDs in primary health care, health systems should address integration of governance, financing, workforce development, service delivery, essential medicines and technologies and health information. Integration in all domains is needed to ensure health systems put people at the center of health care and thus achieve health for all." 83

Overall, the literature identifies a review of existing national health policies to evaluate progress toward integrated care and ensure effective integration on the following topics: develop context-specific evidencebased practice guidelines; establish a high-level multisectoral commission on NCDs with clear guidance to address planning and implementation; ensure resource allocation and training for a sustainable, competent health workforce to manage NCDs and integration; incorporate NCD services at all levels of health care with a specific focus on primary and community care services; invest in research and technology supporting integration of NCD strategies and programs; and include assessments of health and economic impact. 84

The current way of health care provision for chronic conditions is based on a costly and hospital-centered health system and the current implementation carries the risk of quickly losing its connection with population health. The wider determinants of health are also often overlooked. ${ }^{85}$ These determinants include social determinants such as education and wealth as well as advances in information and mobile technologies, nanoscience, and diagnostics. Complex patient conditions and multi-morbidities can no longer be effectively addressed through traditional approaches that focus on individual diseases. Therefore, integrated care necessitates the introduction of new models of care delivery while shifting the focus toward population health management and proactive and preventive care. ${ }^{86}$

Policy makers might consider making strategic entries to the integrated care system through the development of regulatory frameworks. One such framework is on population health management to improve care outcomes and the experiences of care. Integrated care policies for population health management may address issues such as risk stratification, pooling of budgets between health care and social care, health registries, population needs assessment, and identification of high-risk patients. Another regulatory framework is on educational and professional reforms for health care professionals to develop new skill sets and competencies and to improve inter-professional education as well as task delegation within the health workforce. A third possible regulatory framework can be developed for collaborative entities ${ }^{87}$ and care teams to improve care coordination, improve

\footnotetext{
83 WHO 2018.

84 WHO 2018.

85 Sharfstein 2014.

86 Borgermans and Devroey 2017.

${ }^{87}$ A 'collaborative' is defined as a group of independent yet aligned hospitals or health systems that form a legal partnership in pursuit of common goals and initiatives.
} 
integration of medical and social/mental/community care, improve relationships with health professionals and make them more continuous, use evidence-based medicine, and make continuous discharge planning. ${ }^{88}$

One good example for a strategic entry of regulatory frameworks through population needs assessment is the Basque Country's integrated care strategy which reflects the agenda for the health system transformation to address the changing population needs with a particular emphasis on chronic conditions, and which aims to make a shift from a reactive to a proactive health system. The actions of the Basque Country Strategy are therefore focused on issues of (a) population health management, risk stratification, and targeting of population; ${ }^{89}$ (b) prevention and promotion; and (c) continuity of care with the introduction of advanced nursing competencies ${ }^{90}$ through assigning new roles to nurses and creation of integrated care organizations. ${ }^{91}$

A second example is the Netherlands' Zorg In Ontwikkeling (Zio) program which is an integrated care network aiming to shift NCD care from hospitals to the primary level. Started in Maastricht in 1996 as a research initiative aiming to improve health outcomes for diabetic patients, the Zio program had a guiding role for the Dutch MoH in shaping national NCD policy reforms. In 2010, the Netherlands adopted Zio's disease management model as the national model for managing NCDs. For the Netherlands' Zio program, as part of the diabetes disease management program, the regulatory framework allows general practitioners to use condition-specific parameters consistent with Dutch guidelines for diabetes to stratify individuals according to the disease severity. Therefore, the intensity of care depends on each patient's level of need. ${ }^{92}$

\subsubsection{CURRENT STATE IN TURKEY}

The MoH Strategic Plan 2019-2023 focuses on the population needs assessment, risk stratification, high-risk population identification, and education and professional reforms. To this end, the plan highlights the need to strengthen PHC and increase its effectiveness within the health system. The plan indicates the MoH's intention to carry out NCD screening at PHC institutions on HT, DM, and CVD risk assessment and to increase PHC staff competencies through effective FM training schemes that are redesigned and improved to reflect effective NCD management at the primary level. Additionally, the plan indicates designing and standardizing PHC processes where all steps are included and improving patient experience. This way the Strategic Plan aligns with the strategic entries to be made by regulatory framework development, as indicated in the previous section.

To set the legislative background for integrated care, the $\mathrm{MoH}$ has enacted several communiques and directives on CHCs and Affiliated Units, FM Practice and Payment Modalities, HLCs, and Health Literacy. ${ }^{93}$ There are also

\footnotetext{
https://www2.deloitte.com/content/dam/Deloitte/us/Documents/life-sciences-health-care/us-Ishc-providercollaborativ.pdf.

88 Borgermans and Devroey 2017.

${ }^{89}$ Risk stratification helps identify the future care requirements of individuals and analyze the complexity and comorbidity levels within the population. This way, it is possible to proactively design and implement targeted interventions to population segments with different health profiles (Solinis et al. 2013).

90 One example for the new nursing roles is the nurse of advanced competencies who is responsible for the management of complex cases. This function coordinates, manages, and unifies health care for complex patients and provides advanced and personalized care for home-based vulnerable patients (Orueta et al. 2013).

91 Solinis et al. 2013

https://www.interregeurope.eu/fileadmin/user upload/tx tevprojects/library/file 1536224467.pdf.

92 Hubertus et al. 2017.

93 Community Health Centers and Affiliated Units Regulation 2015, Family Medicine Implementation Regulation 2013, Family Medicine Payment and Contracting Regulation 2010, Health Literacy of Population Regulation 2010.
} 
protocols signed with other ministries or institutions on obesity control. ${ }^{94}$ However, integration between the levels of care is not clearly and thoroughly defined by the regulations. The Healthy Living Centers Communique of 2016 and the informative letter sent to provincial directorates in 2018 on the roles and responsibilities of case coordinators of the HLCs are examples of a few and dispersed regulation on integration within primary and preventive care.

From the information systems' perspective, current HIS and the recent studies ${ }^{95}$ support plans for population health management, especially at the primary care level. Family physicians have a registered population, and they are able to plan ahead and follow up as they can have patient lists with certain conditions for their registered population. However, public hospitals cannot have such patient lists because they do not have a registered population. At the local level, there are good practices that can be scaled up: for example, Bursa Osmangazi HLC does the planning for certain NCD cases including the lists of patients who follow HLC services of dietitian and physiotherapist and whose hemoglobin $\mathrm{A} 1 \mathrm{c}(\mathrm{HbA1c})$ value is prediabetic and diabetic. These lists help the HLC staff to follow up with the patients mostly from a patient education and follow-up perspective and not from a treatment perspective. Bursa Osmangazi HLC also strategized and developed plans for future needs and sustainability modeling of the HLC operations. The HLC also defined its technology needs to support operations.

\subsubsection{RECOMMENDATIONS ON INTEGRATION WITHIN PRIMARY CARE LEVEL}

Develop regulations based on the Strategic Plan objectives. The strategic plan includes all necessary objectives and targets on integration that are discussed in literature and implemented in other countries. Enacting necessary secondary legislation (which will concretely define the implementation, coordination, and communication steps between all involved institutions and units) based on these objectives will ensure efficient integration at all levels of health care, specifically focusing on NCDs.

Rewarding compliant behavior. The Turkish government may also consider rewarding compliant behavior of the population. Citizens regularly conducting their health checkups and following the recommendations of health workers on healthy living (diet, exercise, tobacco cessation, and so on) can be given tax rebates on their health premiums or can be subject to lowered payment brackets. Tax rebates can be calculated based on the health history of the citizens or on predicted average health care costs per person.

\subsubsection{CREATING AN ENABLING ENVIRONMENT}

\subsubsection{OVERVIEW AND COUNTRY EXAMPLES}

One of the strategies for integrated people-centered health services is to create an enabling environment. ${ }^{96} \mathrm{An}$ enabling environment should bring together all involved stakeholders to assume the transformational change. A number of different and multifaceted processes are to be pursued to produce required changes in the legislation, financing arrangements, incentives, reshaping of the workforce, and public policy making. ${ }^{97}$

\footnotetext{
94 Protocol with the Ministry of Interior General Directorate of Security 2014.

95 The head of GD Chronic Diseases indicated that the MoH has appropriate disease data and information to plan ahead. What she refers to is the MoH's Burden of Disease Study of 2016.

96 https://www.who.int/servicedeliverysafety/areas/people-centred-care/en/ (accessed December 26, 2018)

97 https://www.who.int/servicedeliverysafety/areas/people-centred-care/five-strategies/en/ (accessed January 14, 2019)
} 
Primarily, a change of culture in the clinical and management practices is needed to establish a shared vision and avoid/reduce problems of sustainability of integrated care. ${ }^{98}$ Second, to ensure alignment among all the processes and actors involved, top management's strong and visible support for the integrated care efforts as well as the existence of joint decision-making mechanisms are crucial. A third enabler is the clinical leadership that is needed to effectively communicate a clear vision on how integrated care can produce required improvements. ${ }^{99}$ The existence of shared accountability through reliable information sharing is also essential because integrated care delivery necessitates the existence and acceptance of clearly defined shared values. ${ }^{100}$

For Germany's Gesundes Kinzigtal scheme, the success behind the model can be explained with a number of enablers. One was the existence of joint management practices and a strong culture of working together, both of which were reflected to the actual practice. ${ }^{101}$ Success of integrated care depends on rather long-term plans clearly showing the support and funding for the integrated care efforts where financial incentives are focused on integrated pathways and designed to be redistributed to stakeholders. ${ }^{102}$ To this end, the program had sufficient time to design, implement, observe, refine-modify, and demonstrate impact. The long-term contracts enabled the Gesundes Kinzigtal scheme to achieve and demonstrate initial results after the initial investment. ${ }^{103}$ The Gesundes Kinzigtal scheme was also able to show its economic sustainability through the introduction of the shared health gain approach and the corresponding shared savings contracts. ${ }^{104}$

The Gesundes Kinzigtal scheme also adequately addressed the regulatory and cultural challenges that would adversely affect the implementation of integration programs. ${ }^{105}$ On the regulatory side, the passing of the Statutory Health Insurance Modernization Act in 2004, aiming to reduce system fragmentation, provided the opportunity to act for the initiators of the model. One component of the Modernization Act allowed health insurance companies to spend up to 1 percent of doctor and hospital expenditures on integrated care programs. ${ }^{106}$ This funding window helped design the shared savings model. On the cultural side, the model introduced the shared decision-making model promoting patient self-management, patient engagement, patient coaching, and follow-up care to address the cultural inertia on the demand side of the health care.107

Extensive implementation of information technology, continuous and rigorous program monitoring evaluation, population risk stratification, customizing of interventions for relevant populations, and ensuring that the

\footnotetext{
98 Maruthappu, Hasan, and Zeltner 2015.

99 Carter et al. 2011.

100 Maruthappu, Hasan, and Zeltner 2015.

101 https://www.local.gov.uk/sites/default/files/documents/kinzigtal-funding-flows-a-3fc.pdf.

102 https://www.tandfonline.com/doi/full/10.1080/23288604.2015.1077301.

Maruthappu, Hasan, and Zeltner 2015.

103 https://www.gesundes-kinzigtal.de/wp-content/uploads/2017/01/Global-Forum GesundesKinzigtal_Case_study_2016.pdf.

104 Hildebrandt et al. 2010.

https://www.ncbi.nlm.nih.gov/pmc/articles/PMC2914874/pdf/ijic2010-2010046.pdf.

105 https://www.tandfonline.com/doi/full/10.1080/23288604.2015.1077301.

Maruthappu, Hasan, and Zeltner 2015.

106 https://www.gesundes-kinzigtal.de/wp-content/uploads/2017/01/Global-Forum_GesundesKinzigtal Case study 2016.pdf.

107 https://www.local.gov.uk/sites/default/files/documents/kinzigtal-funding-flows-a-3fc.pdf.
} 
themes of prevention and public health set the background of all efforts under the program were among the other success factors for the Gesundes Kinzigtal. ${ }^{108}$

Similar factors facilitated the integrated care efforts in Basque Country. First, rising burden of NCDs was a driver for change in the Basque Country. The 'Strategy for Tackling the Challenge of Chronicity in the Basque Country' launched in 2010 by the then new government set the vision to transform the health system to better respond to the prominent NCD issues and to make a shift from a reactive to a proactive health care approach through integrated care efforts. Second, the existence of a strong primary care system and high patient satisfaction at the primary care level were advantages for integrated care initiatives, and there was additional need to address the problems of a lack of continuity and coordination. The reform process aimed to introduce a patient-centric model through a population health management perspective. With the help of population risk stratification, identification of individual patient requirements, and analysis of comorbidity in the population, the program aimed to facilitate the design and implementation of interventions for specific patient groups. Third, funding mechanisms are revisited and transformed to reward good performance and achievement of results. To this end, the Contract Program of 2012 linked 3 percent of the budget for the public health care providers to the achievement of a number of pro-integration objectives in the same operational area and same population. A fourth factor was that prevention and health promotion were the underlying themes of the strategies being implemented and allowed the policy makers to include population- and community-based approaches into the model. Finally, the systematic use of assessment schemes to track the implementation progress as well as the health outcomes helped the stakeholders analyze the results at both patient and population levels and make evidence-based decisions to further shape the integrated care initiatives. ${ }^{109}$

In Japan, a suitable economic context helped introduce change in the health care model toward integrated care. The presence of universal health insurance since 1961 and a mandatory long-term care insurance system since 2000 allows all Japanese citizens to access long-term care at a minimal cost. This arrangement presented a viable economic framework to integrate medical care and long-term care and allowed the government to initiate community-based integrated care centers nationwide in 2005 while also revising the Long-term Care Insurance Act. The Mitsugi model eventually became the basis of national policy in 2012, and it is expected that the model will be implemented nationwide by 2025.110

The success of the Mitsugi case was also an outcome of a responsible and innovative leadership which had a critical role in uniting the stakeholders. Acquiring the political support from the local and national governments was another driver of success in the Mitsugi case. ${ }^{111}$

\subsubsection{CURRENT STATE IN TURKEY}

There are context-specific local enablers in the Turkish case which might provide insight and guidance to efforts to expand and scale up future integrated care efforts. The two examples below are on the successful HLC operations.

Bursa Osmangazi HLC is successful for a number of reasons. First, there exists a vision and a will to operate HLCs in Bursa. The Bursa Provincial Health Director ${ }^{112}$ has been a health care manager for 10 years, which

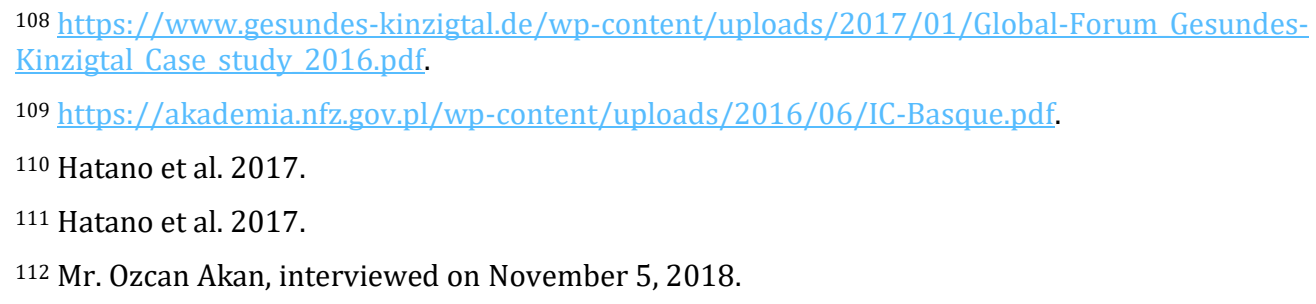


helped sustain the HLC vision to strengthen primary care. The Provincial Director believes that NCDs need to be primarily addressed at the primary care level. Second, the use of MoH-owned buildings for HLCs in Bursa helped the teams to be ahead of the process. Third, Bursa Provincial Health Directorate's good relationship with the local government was a good leverage to find convenient venues for HLCs that would allow easy access of patient. A fourth factor of success is that the Bursa Osmangazi HLC responsible physician is a public health expert and this facilitates planning the HLC operations and making them more effective. Bursa Provincial Health Directorate was also successful in collecting data and evidence not only for operational monitoring and evaluation but also for using this evidence to make the case for additional need of HLCs in the province.

In Hatay, the coordination model for the condition of thalassemia is a major factor that draws patients to primary care and to HLCs. Because thalassemia tests for couples to be married are mandatory in Hatay and these tests are carried out in HLCs, those couples who visit HLCs already know about the services provided in those centers.

3.2.2.3 RECOMMENDATIONS ON INTEGRATION WITHIN PRIMARY CARE LEVEL

Establish a committee or consortium focused on best practices. It is recommended that the Minister of Health establish a formal clinical advisory structure (a committee or a consortium). The clinical advisory group would specifically focus on Turkey's mission to implement an integrated care model optimizing NCD and prevention management. This group would monitor the implementation of the integrated care strategy. Representatives appointed to serve in the committee would include national and local leaders and subject matter experts that can help design and implement the initiative. The committee's function would support horizontal (peer to peer, district/province) and vertical (local to national) communication, provide feedback to promote culture change, identify and promote opportunities for sites to share best practices, and provide perspective on barriers/challenges all of which would inform the Minister of Health as guidance, policy, and regulations are developed. One of the key steps in effecting large delivery system changes is convening a committee to address integration efforts.

Specific steps to accomplish establishing a clinical advisory group to enhance implementation and communication efforts between the field and the MoH include the following: (a) charter a workgroup with specific goals, objectives, timelines, deliverables, and measures/monitoring; (b) authorize clinical/administrative champions and leaders to participate and lead the effort; (c) address resource/financial and/or policy issues in support of initiative; (d) provide support for staff/training and time commitment to implement; (e) develop and distribute a stakeholder communication plan; and (f) establish regular meetings to receive updates from project leaders, review/monitor progress, celebrate success, and understand/address challenges and barriers.

Increase communication, socialization, and marketing strategies. Communication, socialization, and marketing to all stakeholder groups are cornerstones of successful implementation and change efforts. The $\mathrm{MoH}$ may review and enhance efforts aimed at building understanding, acceptance, and participation for change under the broader integrated care strategy. Topical areas include the need to engage patients in participating in their health and well-being with the support of the FMC and HLC staff. Health care leaders at all levels should receive important information and updates on goals, objectives, progress, and so on, to foster buy-in and participation. Targeting other groups that are not an integral part of the FMC/HLC integration plan but may be important actors in future next steps for system integration paves the way and engages other levels of care at the onset. For example, communicating with specialists to keep them informed of the integration of FMC/HLC to address NCDs and prevention care will support future efforts to shift low-complexity care from hospitals to FMC/HLC resulting in improvements of care across different levels. Targeting efforts may include developing key messaging for specialists to use with patients as they discharge them back to FM care, ensuring 
that negative financial impacts to hospitals/specialists are addressed, developing formalized communication/work process-based agreements that are mutually developed between the FMC and specialists, supporting integrated care technology systems development and implementing to support referrals and counter-referrals follow-up.

As a specific action for HLCs, there needs to be a strong political commitment and visible official support for HLC operations both at the central and local levels. This will help increase and sustain awareness for HLCs and their functions.

\subsubsection{ALIGNMENT OF FINANCING AND INCENTIVES}

\subsubsection{OVERVIEW AND COUNTRY EXAMPLES}

According to the literature, incentives can be monetary and non-monetary in nature and the literature indicates that both are effective in promoting change. ${ }^{113}$ The literature regarding alignment of finances and incentives for care integration is expansive but presents significant variation in identifying the best models as system and country contexts differ. Topics covered include financing and incentives options such as investments, business case, reward schemes (for example, performance based financial bonuses), pooled budget, shared risk and revenue/profits, bundled payments, capitated payments, long-term contracts, and end-to-end managed care.114 As country contexts and requirements vary, incentivization schemes and health care system financing require adjustments and alignment at the local, regional, and national level to be effective. ${ }^{115} \mathrm{~A}$ crucial element for success is the removal of inhibitors, both legal and financial constraints that may affect progression toward integrated care goals. The WHO's 2010 World Health Report indicates that pay for performance introduced separately and independently in different programs can result in competitive performance incentives, with each program vying to get providers to do providers' work rather than that of other programs. ${ }^{116}$ Evidence from the EU indicates that pay-for-performance formulae focusing on clinical quality and patient experience were associated with greater improvements in care coordination and office staff communication, while a focus on productivity and efficiency had a negative impact on communication and, consequently, patient care. Appropriate distribution of these funds across health care teams is also an important factor to avoid tensions and issues that may undermine working relationships. ${ }^{117}$

In general, provider payments should be designed to encourage performance improvement for providers to improve the experience of care, improve the health of populations, and reduce costs. Incentives and payment methods need to be aligned with system goals, be consistent across providers/payers, and address provider protection from unavoidable risk as well as variation in patient morbidity. ${ }^{118}$

Literature also suggests that team members have more satisfaction from team-based performance incentives than individual incentives. Team-based incentives and feedback systems are being used by a number of integrated health system schemes including Kaiser Permanente, Virginia Mason, Geisinger Health System, and

\footnotetext{
113 Brook 2015. https://www.rand.org/pubs/corporate pubs/CP788.html.

114 European Union 2017.

115 WHO 2016b.

116 WHO 2010a.

117 European Union 2017.

118 Borgermans and Devroey 2017.
} 
the Massachusetts General Hospital. These incentives helped achieve improvements in process outcomes, such as screening rates and adherence to protocols. ${ }^{119}$

Concerning the alignment of finances toward integrated care, the literature and global experience indicate that estimating the costs and benefits of an investment strategy to prevent and control NCDs is imperative. ${ }^{120}$ Routine analysis and publication of the return on investment (ROI) is critical to reassure the population and staff that investments in the public health and health care system are working and worthwhile. A recent paper in the Lancet ${ }^{121}$ describes a framework gleaned from in-depth review and validation using the OneHealth Tool $^{122}$ (WHO SDGs) to estimate the costs of scaling up a set of interventions to control the increasing burden of and to reduce premature morality associated with CVD. The methodology was applied to 20 countries with the highest NCD burden. The study included not only the cost of health care treatment/investment impact but also the social burdens/benefits experienced such as reduced employment and productivity (loss of household income, economic output, and so on) and social benefits of increased years of healthy life. The study on the analysis of delivering limited set of interventions for cardiovascular care (tobacco, sodium reduction, and pharmaceutical interventions) in 20 high-, middle-, and low-income countries demonstrated a significant potential for immediate health and economic benefits averting up to 13 million stroke incidents and 8 million ischemic health disease incidents within 15 years investment of US\$1.50 per capita per year. Another systematic literature review published by Elsevier Inc. in 2016 noted predominantly positive economic impacts of integrated care models for patients with chronic diseases. ${ }^{123}$

\subsubsection{CURRENT STATE IN TURKEY}

The MoH Strategic Plan 2019-2023 indicates that the current FM performance payment system needs to be updated. The plan sets forth the intention to do so under Goal 2: Strengthening PHC and increase its effectiveness within the health system. One of the strategic design features of this goal is about redesigning the FM performance system to be more effective and to include elements such as quality, satisfaction, and resultsbased focus and to reflect the $\mathrm{MoH}$ priorities. In that sense, it highlights the need to make the performance system consistent with the changing roles of the primary care system concerning NCDs. However, current performance-based contracts do not address NCDs but target negative incentives for MCH. Local leaders and clinical staff are therefore required to focus on these goals that reduce and limit concurrent focus on NCD management. Revising the performance-based contracts to include NCD management and maintaining MCH achievements have been in the agenda of the MoH for many years but could not be initiated as the MoH could not get into a mutual agreement with other ministries and the Strategy and Budget Unit of the Presidency.

Being aware of the significant increase of NCD disease burden, the MoH was also active in making use of the WHO's OneHealth tool and the General Directorate of Public Health conducted a study using this tool ${ }^{124}$ which supports the business case for future gains and ROIs. ${ }^{125}$ This study suggests that efforts for NCD prevention are relatively cheap and cost-effective. Costing and ROI analysis of the five interventions defined within the scope

\footnotetext{
119 Blumenthal et al. 2013.

120 Bertram et al. 2018.

121 Bertram et al. 2018.

122 https://www.who.int/choice/onehealthtool/en/.

123 Desmedt et al. 2016.

124 WHO OneHealth Tool.

https://www.who.int/choice/onehealthtool/OneHealth Tool Supporting integrated strategic health planning.pdf?ua=1.
} 125 WHO, MoH, and UNDP 2018. 
of the study (policy interventions for tobacco and alcohol use, salt intake, and physical inactivity, and clinical interventions for CVD and diabetes in primary care) reveals that the economic benefits of these interventions exceed their implementation costs in both the shorter and longer time periods (5 years and 15 years, respectively). The $\mathrm{MoH}$ can repeat such studies to inform decision makers on the financial returns of NCD interventions in the medium to long term.

\subsubsection{RECOMMENDATIONS ON INTEGRATION WITHIN PRIMARY CARE LEVEL}

This report acknowledges that all the recommendations listed in the following paragraphs have financial implications, some are directly attributed to payment schemes, and others are directly tied to improving the infrastructure needed to manage NCDs and prevention care to achieve integrated care outcomes. It is believed that implementation of the proposed recommendations will positively affect the aspirations set forth in the MoH Strategic Plan, in both the short and long term.

Update performance-based contracts. Incorporating the positive incentive scheme for NCDs to the current performance payments would be a complex task but certainly achievable given the MoH's expertise in this area. As discussed earlier, published literature provides ample evidence that supporting pay for performance formulae focusing on clinical quality and patient experience is associated with greater improvements in care coordination and communication.

Develop and implement team-based incentives. As discussed earlier, the literature also suggests that team members have more satisfaction from team-based performance incentives than individual incentives. Such incentives and feedback systems can be developed to achieve improvements in process outcomes, such as screening rates and adherence to protocols. The $\mathrm{MoH}$ may consider introducing team-based incentives as team effectiveness appears to be highest when multiple individuals need to work together to complete a task; team rewards and performance evaluations can motivate team members to work together more effectively. Conversely, individual performance assessments and compensation for work requiring a team can undermine team effectiveness and impede team performance. To keep the motivational effects of team-based rewards, these mechanisms need to be supported by team training and performance feedback systems. Incentives improve team performance when team members understand the concepts of building effective teams, being good team members, and when they see their progress toward a concrete goal. It is also important to keep the rewards and performance evaluation systems simple to the extent possible as complex systems let employees lose sight of the links between their work and the rewards, thus eroding the motivational power.

Develop a ROI evaluation system. The MoH may develop and establish a routine ROI evaluation modality into its programs. The evaluation reports and the findings should be regularly shared with other ministries and the Strategy and Budget Unit of the Presidency to inform them on the savings that would be attained in the future.

\subsubsection{MONITORING AND PERFORMANCE IMPROVEMENT}

\subsubsection{OVERVIEW AND COUNTRY EXAMPLES}

Measuring baseline and project progress is a critical element in implementation. Leadership and staff alike need to have quantitative and qualitative data to inform and plan as the integrated care efforts ensue. The availability of key data and performance metrics at all levels of a health care organization known as data transparency is a crucial element of performance improvement. Key stakeholders and frontline delivery staff need data and information to monitor and adjust their progress toward identified goals. The literature supports

this approach, "the collection and dissemination of data to care providers is a means to support them in 
exploring opportunities for continuous improvement of health care provision."126 There are many examples of national, regional, and local health care delivery measures that have been used effectively around the globe to monitor progress toward integration, NCD and prevention care management, teamwork, and so on ${ }^{127}$ (metrics references). ${ }^{128}$

Much of the literature categorizes integrated care measures into three areas: (a) structural measures that are precursors of integration; (b) process measures that inform regarding intermediate outcomes or internal process variables, which assess the level of system-wide activity and are the means to achieving ultimate goals; and (c) outcome measures that assess how a system is fulfilling its ultimate purpose. Both patient and cost measures (mortality, morbidity, functional status, costs) are the end goal for improvements in care coordination. ${ }^{129}$

Metrics (or performance indicators) should include measurement in clinical quality (NCD clinical indexes, emergency department [ED] visits, Ambulatory Care Sensitive Condition [ACSC] hospital admissions); patient experience (access, continuity, satisfaction with care, improved team relationships); staff experience and satisfaction (high performing teams - less burnout, defined roles and functions-improved efficiency, improved teamwork and comradery); and cost-effectiveness (ROI, shift of hospital care to lower levels, and so on).

ACSCs are described in several papers from Germany, Portugal (WHO Europe), and the United States. ${ }^{130}$ These metrics are extracted from data on hospital admissions for NCD diagnoses and are considered proxy measures for the effectiveness of the PHC system in managing NCDs. Theoretically, admission for these conditions is minimized by good outpatient PHC that can potentially prevent the need for hospitalization or for which early intervention can prevent complications or more severe disease. ${ }^{131}$ Examples of ACSC admission indicators include diagnoses of diabetes, HT, COPD, and urinary tract infections admission rates. ${ }^{132}$

Countries seeking to transform health care to achieve people-centered, integrated services are developing and adapting a set of quality indicators through which system performance can be monitored. Increasingly widely used in low, middle, and advanced economies, indicators for quality, cost, efficiency, patient, and provider satisfaction provide valuable information at the national, regional, and local levels on which policy, funding, governance, and performance are monitored and measured. For example, Japan, the United Kingdom, New Zealand, and the United States have developed sets of indicators that monitor progress toward improved quality care for the populace served.

The United Kingdom has 35 specific measures over 6 key domains that measure the quality of integrated care. The domains include Community Well-being and Population Health, Organizational Processes and Systems,

\footnotetext{
126 Hubertus et al. 2017; WHO 2013.

127 Agency for Healthcare Research and Quality. Care Coordination Measures Database. Clinical Community Relationship Measures. Team Based Primary Care Measures Database. https://primarycaremeasures.ahrq.gov/.

128 Davis et al. 2014; Schultz et al. 2013; Social Care Institute for Excellence 2017.

129 Strandberg-Larsen et al. 2009.

130 Agency for Healthcare Research and Quality. Prevention quality indicators overview. https://qualityindicators.ahrq.gov/Modules/pqi_resources.aspx.
}

WHO Europe 2015, 2016.

131 Agency for Healthcare Research and Quality. Prevention quality indicators overview. https://qualityindicators.ahrq.gov/Modules/pqi_resources.aspx.

132 Agency for Healthcare Research and Quality. Prevention quality indicators overview. https://qualityindicators.ahrq.gov/Modules/pqi_resources.aspx. 
Personal Outcomes, Resource Use/Balance of Care, Service Proxies for Outcomes, and User/Carer Experience. Quantitative and qualitative data are used to generate a scorecard at the national, regional, and local levels. Indicators' development occurred in a phased approach of selection, and indicators were developed for the short term-maximizing the potential of existing data sources from those routinely available in the systemand the medium to long term-developing additional, more specific indicators from new analyses of existing data sources and identifying gaps in data needs for future development and/or execution/implementation as the system becomes more sophisticated. ${ }^{133}$

In New Zealand, an integrated performance and incentive framework was drafted in 2013 containing an inventory of measures to support District Health Boards to identify and use locally relevant system-level measurements indicating progress toward care integration and improved health and equity for all population groups. ${ }^{134}$ The metrics not only support monitoring in the usual domains such as mortality for cardiovascular, diabetes, infections, smoking, vaccinations, and so on but also monitor ACSC admissions, leadership: capability and capacity to use resources, ensuring efficiency and effectiveness, patient experience, and access to health care.

The VHA ${ }^{135}$ has a long history of driving change through measurement. As noted in the WHO paper, Integrated Care Models (October 2016), the VHA is a highly integrated, publicly funded health care system where service integration and shared accountability is closely monitored, measured, and transparently reported to the public. ${ }^{136}$ The VHA also developed and published reports for clinical staff as well as administrators. The report for primary care clinical staff, known as the Compass, was developed as a guide so teams could view data on important process, structure, and outcome metrics for access, quality, and patient satisfaction in nearly real time to understand their progress toward critical goals. The reports display data at the team, outpatient clinic, region, and national levels. They are accessible by any VHA employee and/or authorized contractors. The ability to view others' data and compare each primary care team promotes friendly competition and sharing of best practices locally. Local, regional, and national leaders and administrations view specially designed scorecards (Table 4) that roll up the data and also allow drill down to the individual primary care teams. This level of report and drillable information enables leaders to target teams or regions for performance improvement efforts.

\footnotetext{
133 Raleigh et al. 2014.

134 Description and outline of potential measures. Draft integrated performance and incentive framework (IPIF). New Zealand: Health Improvement and Innovation Resource Framework 2013 (http://www.hiirc.org.nz/page/42610, February 7, 2019).

135 The VHA is the largest vertically and horizontally integrated care network in the United States, providing care at 1,250 health care facilities, including 172 medical centers and 1,069 outpatient sites of care of varying complexity (VHA outpatient clinics), serving 9 million enrolled veterans each year. https://www.va.gov/health/index.asp.
}

136 Access and Quality Veterans Health Administration Public Report website. https://www.accesstocare.va.gov/. 
Table 4: VHA Leader Scorecard for Primary Care

\begin{tabular}{|c|c|c|c|c|c|c|}
\hline Regions & GP/Staff Ratio & $\begin{array}{c}\text { Access within } \\
7 \text { Days }\end{array}$ & $\begin{array}{l}\text { Same Day } \\
\text { Access }\end{array}$ & $\begin{array}{l}\text { Same GP } \\
\text { Continuity }\end{array}$ & $\begin{array}{c}\text { Contact 2Days } \\
\text { Post DC }\end{array}$ & $\begin{array}{l}\text { Telephone } \\
\text { Encounters }\end{array}$ \\
\hline V01 & 3.06 & $93.39 \%$ & $58.80 \%$ & $77.32 \%$ & \begin{tabular}{|l|}
$\mathbf{5 7 . 9 2} \%$ \\
\end{tabular} & $25.92 \%$ \\
\hline V02 & 3.01 & $92.05 \%$ & $69.12 \%$ & $80.85 \%$ & $54.68 \%$ & $24.60 \%$ \\
\hline V03 & 3.03 & $92.23 \%$ & $66.23 \%$ & $78.10 \%$ & $59.51 \%$ & $30.22 \%$ \\
\hline V04 & 2.75 & $95.61 \%$ & $76.46 \%$ & $81.59 \%$ & $59.85 \%$ & $29.33 \%$ \\
\hline V05 & 3.00 & $87.52 \%$ & $60.67 \%$ & $72.80 \%$ & $47.91 \%$ & $26.56 \%$ \\
\hline V06 & 3.26 & $85.45 \%$ & $58.38 \%$ & $71.63 \%$ & $57.25 \%$ & $26.78 \%$ \\
\hline V07 & 3.16 & $89.63 \%$ & $64.55 \%$ & $75.44 \%$ & $54.56 \%$ & $28.77 \%$ \\
\hline V08 & 2.92 & $90.33 \%$ & $70.07 \%$ & $77.54 \%$ & $54.36 \%$ & $29.92 \%$ \\
\hline V09 & 3.32 & $90.58 \%$ & $61.85 \%$ & $73.41 \%$ & $63.88 \%$ & $25.38 \%$ \\
\hline V10 & 3.51 & $94.04 \%$ & $78.13 \%$ & $77.93 \%$ & $59.47 \%$ & $25.83 \%$ \\
\hline V11 & 3.38 & $93.66 \%$ & $59.16 \%$ & $77.20 \%$ & $59.06 \%$ & $29.86 \%$ \\
\hline V12 & 3.00 & $93.11 \%$ & $72.46 \%$ & $75.19 \%$ & $58.95 \%$ & $30.62 \%$ \\
\hline V15 & 2.80 & $91.86 \%$ & $62.64 \%$ & $77.75 \%$ & $45.23 \%$ & $30.35 \%$ \\
\hline V16 & 3.21 & $94.33 \%$ & $79.67 \%$ & $80.01 \%$ & $56.68 \%$ & $26.61 \%$ \\
\hline V17 & 3.16 & $92.47 \%$ & $73.56 \%$ & $77.79 \%$ & $57.87 \%$ & $32.60 \%$ \\
\hline V18 & 3.51 & $88.69 \%$ & $50.43 \%$ & $78.01 \%$ & $49.06 \%$ & $25.42 \%$ \\
\hline V19 & 2.66 & $81.96 \%$ & $37.70 \%$ & $77.49 \%$ & $51.12 \%$ & $30.14 \%$ \\
\hline V20 & 3.13 & $84.40 \%$ & $49.26 \%$ & $80.13 \%$ & $65.54 \%$ & $27.85 \%$ \\
\hline V21 & 3.01 & $87.51 \%$ & $49.84 \%$ & $74.81 \%$ & $59.28 \%$ & $22.33 \%$ \\
\hline V22 & 3.30 & $92.43 \%$ & $64.79 \%$ & $72.48 \%$ & $46.04 \%$ & $20.99 \%$ \\
\hline V23 & 3.07 & $87.47 \%$ & $53.84 \%$ & $78.63 \%$ & $54.34 \%$ & $32.25 \%$ \\
\hline
\end{tabular}

Another innovative example is the transparent monitoring and evaluation case from Afghanistan where the Ministry of Public Health has been using a balanced scorecard model since 2004 to drive quality improvements for providers and health care sites and later making the data available to communities. ${ }^{137}$ The scorecard displays 6 domains on Patients and Community, Staff, Capacity for Service Provision, Service Provision, Finances, and Overall Vision and 29 indicators for these domains. This model aims to use the monitoring system framework to identify priority areas for improvement, and data demonstrate that substantial improvements have been achieved over time when compared to the baseline period of 2005-2006. Improvements include increases in "median provincial scores for presence of active village health councils, availability of essential drugs, functional laboratories, provider knowledge, health worker training, use of clinical guidelines, monitoring of tuberculosis treatment, and provision of delivery care."138

Finally, the Japanese case is a good example to show how integrated systems produce positive results and health outcomes. Mitsugi's community-based integrated care initiative has brought about three significant results, all tracked through regular measurements. First, the initiative helped reduce the number of bedridden people in town as the new integrated model prevented patients from inadequate post-acute home care through home-based care service provision and strong care collaboration among health care specialists. Second, the increase of health care costs has slowed down since the home-based specialist care prevented several common conditions among the bedridden patients. Third, the number of people having medical checkups increased, possibly because of public health promotion and awareness campaigns conducted by public health nurses affiliated with Mitsugi Hospital. The nurses were in an advantageous position to link hospital medical services with prevention services. Public health promotion and disease prevention activities also

\footnotetext{
137 https://www.measureevaluation.org/resources/networks/datausenet/dashboards-and-data-use-forum-may2010/Afghanistan\%20Balanced\%20Scorecard\%20-\%20IJHPM.pdf.

138 https://www.measureevaluation.org/resources/networks/datausenet/dashboards-and-data-use-forum-may2010/Afghanistan\%20Balanced\%20Scorecard\%20-\%20IJHPM.pdf.
} 
enjoyed the participation of other health workforce including physicians, rehabilitation experts, and other medical staff. 139

\subsubsection{CURRENT STATE IN TURKEY}

The MoH shows a high-level commitment and intent to address and control NCDs. The MoH Strategic Plan 2019-2023 sets forth the performance indicators and targets through which the progress in PHC delivery, integration of primary care, and NCD management is to be monitored. However, the progress on the achievement of these targets is highly contingent upon the effective introduction and implementation of designed policies and actions for integrated care at the primary level and NCD management.

A set of NCD-related clinical indicators has been developed by the MoH. ${ }^{140}$ Data for some of these indicators are collected through Hospital Management Information System. Examples of indicators include hospital admissions due to diabetes without complications and with acute complications, share of patients whose $\mathrm{HbA1c}$ is greater than 9 on the latest examination, share of patients who have taken diabetes training, share of patients with advance renal disease due to diabetes, and share of patients for whom foot examination is done. While these data are being collected at the hospital level, their connection and coordination with the primary level is currently missing.

Achievement of NCD management targets will highly depend on the formally committed performance of family physicians. But interviewed $\mathrm{MoH}$ officials indicate that such a formal commitment-that is, positive incentives for NCD management (linked with the achievement of targets on screening and follow-up) at the primary care level-is possible only with a decrease in the registered population to family physicians, ${ }^{141}$ and this necessitates an increase in the family physician workforce.

The information infrastructure to keep track of the NCD screening and follow-up activities as well as the actual performance of family physicians for NCD incentive payments is ready but can only be operational if the system is jointly accepted to be used by the Ministry of Treasury and Finance and the MoH.

\subsubsection{RECOMMENDATIONS ON INTEGRATION WITHIN PRIMARY CARE LEVEL}

Incorporate NCD prevention and control into the workload of family physicians through reducing their registered population. As discussed earlier, the actualization and achievement of NCD management and performance targets will highly depend on the formal NCD performance arrangements for family physicians, complemented with an increase in the health workforce and a decrease in the registered population. As the interviewee from the $\mathrm{MoH}$ suggests, a twofold approach is required to increase the workforce figures. One is to encourage primary care practice among medical students as a career preference and the other is to convince the Ministry of Treasury and Finance to increase the recruitment of FMCs.

Provide transparent reporting of critical health care metrics. The $\mathrm{MoH}$ has developed a robust and impressive database of clinical and administrative data from which important decisions are made regarding the design, delivery, and evaluation of health services to the Turkish population. Analyses of health indicators derived from the database provide leaders with invaluable information on which to formulate national strategies, policies, and so on. However, a critical approach to system transformation is data transparency and availability. The MoH should continue to identify and prioritize metrics specifically for PHC services on NCDs

\footnotetext{
139 Hatano et al. 2017.

140 https://dosyamerkez.saglik.gov.tr/Eklenti/3502,gostergeyonetimirehberi-1408151pdf.pdf.

141 Current figure of registered population is more than 3,000 and this needs to be reduced to 2,000.
} 
already available in the database, for patient experience, as well as develop indicators that target integration and goal achievements. Most importantly, data should be available and reportable at each level of the $\mathrm{MoH}$ (national, provincial, and district) and frontline staff to drive change. Metrics should be generated and reported within existing databases versus manual data that are labor intensive and difficult in terms of reliability/validity. There should be data transparency, ensuring availability at each level. Reports should be formatted for intended audiences and drillable to the individual staff member/team. Frontline staff should be able to view their own as well as other teams' data as a means to promote self-monitoring and ownership to achieve goals and to provide a glimpse of other teams' performance to identify and share strong practices.

Use key proxy indicators and process indicators to measure the effectiveness of PHC on NCDs. Progress toward integrated and NCD care including quality of care can be measured through a number of key proxy indicators such as ACSC hospital admissions for the most prominent NCDs such as diabetes, HT, and COPD. ACSC indicators are based on hospital inpatient data; however, they provide insights on the PHC system or outpatient services outside the hospital settings. ACSCs are critical in demonstrating that good and high-quality outpatient/primary care can potentially prevent the need for hospitalization and that early interventions can prevent complications arising from NCDs. To illustrate in practical terms, patients with diabetes may be hospitalized for diabetic complications if their conditions are not adequately monitored or managed at the primary care level and/or if they do not receive the patient education needed for appropriate self-management in the primary care environment. In the Turkish case, ACSC indicators will use data on hospital admissions and hospital discharge, which are currently available in Turkey's robust information system, and when monitored over time, will provide valuable insight into the integration and NCD management in Turkey's FMC/HLC PHC system.

Process indicators that demonstrate necessary steps or workflow for care coordination, care/case management, and so on, are also important in monitoring the PHC system Examples include number of staff attending required training (integrated care, NCD, patient communication interventions, and so on), number and quality of care coordination or service agreements established between FMCs and HLCs, number of support staff assigned to an individual FMP, number of HLC staff assigned per population, number of referrals by FMP to associated HLC, number of patients case or care managed by support staff, and so on. These data may not be available electronically but can be systematically collected at the local level and can be shared with the central units to be analyzed and evaluated for future decisions.

Monitor compliance with NCD clinical practice guidelines (CPGs). Implementation of the National Patient Tracking System NCD modules (UHTS-NCD modules) planned in 2019 will enable point-of-care staff to be guided and to apply evidence-based medicine and CPGs as they care for their patients. Monitoring and transparent reporting at the practice team level on compliance with the guidelines will foster quality improvement and staff participation in NCD management. It is recommended that a monitoring policy and associated guidance be developed jointly with subject matter experts and leaders to be rolled out as part of the employee performance plan. There are a range of approaches to monitoring compliance and adherence to evidence-based practices. Monitoring should be based on an identified set of indicators for a population of patients assigned to each FMP and his/her team. This approach cements the concepts of NCD management, individual and team accountability, and responsibility at the PHC level. Monitoring and evaluation of compliance with NCD CPGs can be conducted through automated information systems or manual systems. Examples of monitoring from automated systems such as UHTS-NCD modules might include NCD health indicators, ACSC admissions, referral patterns to specialist care for care that could have been provided at the primary level, appropriate medications prescribed for each NCD, and specific tasks that support NCD management such as completion and documentation of diabetic foot exams at each visit, annual microalbumin tests, annual lipid monitoring, and documentation of annual retinal eye examinations. Non-automated 
compliance monitoring systems tend to be more labor intensive but are important supplements to automated systems. These include routine peer reviews of documentation of care delivered in accordance with CPGs, observation of care delivery, and so on.

Include integrated care goals in staff performance evaluation system. The $\mathrm{MoH}$ recognized the importance of employee performance and has hired a consultant group to support a review, and revision of the employee performance evaluation system. Employee performance should be directly linked to identified integrated care goals at every level of the health care organization. Data and information systems can support reviews of staff performance and instigate changes and decision making at each level of the system. Factors identified by the $\mathrm{MoH}$ in individual staff performance evaluations such as goals for NCD, prevention, and teamwork place focused accountability and responsibility not only on leadership (national, provincial, and district) but also on care delivery at the point of care (FMC and HLC). Developing specific performance expectations for each level, all aimed at improving health and well-being of patients, improving teamwork, attending and applying standardized training, coordinating and managing care, and so on, will focus staff effort on the work that ultimately leads to system change and improved integration. "For integrated care to operate effectively, collaborative ways of working need to be embedded as 'business as usual' and not seen as additional responsibilities". ${ }^{142}$

\subsection{CARE DELIVERY}

Integrated care delivery has significant impacts on the efficacy and ultimate clinical outcomes of the transformation effort. Care delivery involves engaging the clinical staff in providing care in multidisciplinary teams, understanding how to work within a new delivery model through standardized training, actively participating in coordinating patient care with other providers within the health care system, and using clinical evidence, pathways, and protocols to manage NCDs and preventive care.

\subsubsection{MULTIDISCIPLINARY TEAMS AND STANDARDIZED TRAINING}

\subsubsection{OVERVIEW AND COUNTRY EXAMPLES}

As the systems of care are transformed, many new roles need to be created and new skills need to be developed. Demands for health care continue to change necessitating that the skills, talent, and experience are retained, and the systems of care become 'learning systems' that are constantly striving to improve productivity and increase success. ${ }^{143}$

Establishing a team of health care professionals that care for an assigned group of patients' needs for preventive, chronic, and acute care has emerged as a cornerstone of good PHC practice. ${ }^{144}$ Most importantly, global literature now acknowledges that PHC is the foundation of a stable, cost-effective, and efficient health care delivery system. ${ }^{145}$ Shifting costly, low-complexity care from hospitals to primary care and to community

\footnotetext{
142 Curry et al. 2013.

143 European Union 2017.

144 Bodenheimer et al. 2014; Heslop, Power, and Cranwell 2014.

145 World Health Organization, OECD, and International Bank for Reconstruction and Development/The World Bank 2018.
} 
practices is a critical strategy in addressing overburdened, high-cost hospital systems to reduce costs and improve the health of the populations served. ${ }^{146}$

The literature cautions, however, that shifting care from hospitals to primary care and to community practices requires careful planning and execution to ensure that PHC services have the capacity and capabilities to meet the demand. ${ }^{147} \mathrm{~A}$ key means to meet the burgeoning demand is establishing a team-based approach to deliver PHC where the care of a registered population is shared by a multidisciplinary team of providers with clearly defined roles, responsibilities, and workflows to meet the care needs of the population served. ${ }^{148}$ Developing and maintaining a team-based care approach requires that members are trained, educated, and supported in functioning at the top of their license or scope of practice and are provided with the tools and guidance to effectively deliver care. Well-implemented, integrated team-based care has demonstrated improvement in the comprehensiveness, coordination, efficiency, effectiveness, and value of care, as well as the satisfaction of patients and providers. ${ }^{149}$

Two good country examples of multidisciplinary teamwork are the regional nonprofit organization (NPO) for Diabetic Care in Burgas, Bulgaria, ${ }^{150}$ and the POTKU project (Potilas kuljettajan paikalle, Putting the Patient in the Driver's Seat) in Finland. ${ }^{151}$ Both projects demonstrate that teamwork and integrated care make a difference in the quality, health, and well-being of patients. ${ }^{152}$

The NPO for Diabetic Care in Burgas, Bulgaria was founded in 1994 and provides an integrated approach as well as comprehensive, coordinated primary and secondary care for patients with diabetes and other multimorbidities. The team consists of a nurse, a general practitioner, a cardiologist, and lay people with diabetes and a total of 380 people are functioning in these teams. All members are volunteers providing comprehensive care for patients. The NPO acts a hub of care management and mediator between different professionals and health systems. Access to services is free of charge, irrespective of insurance or socioeconomic status and most patients have a low socioeconomic status, no insurance, and a high morbidity rate. Basic primary care services are provided, and the team supports care coordination with other services. Access to secondary care is also free through cooperation agreements with private hospitals. Cooperation agreements between the NPO and secondary care facilitate the exchange of information among health care providers. Care pathways are jointly developed by all participating providers at different levels of care to support coordination efforts. Patient education and self-management are also emphasized, and the NPO provides training aimed at improving patients' understanding and self-management of their health. Patients also complete a questionnaire about

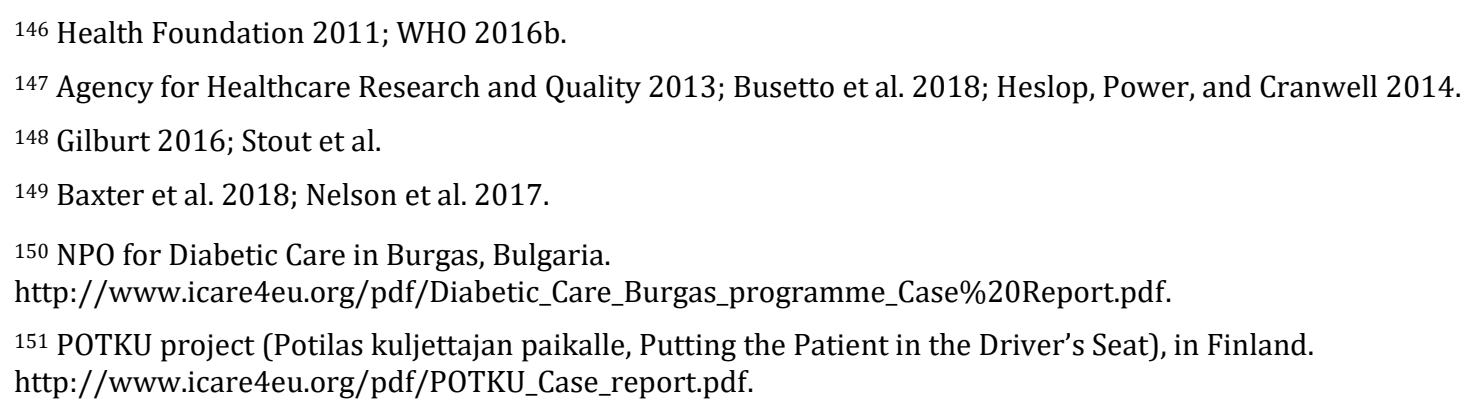


their health to inform staff and support the co-production of personal health goals. Program results include reduction of hospital admissions and amputations from complication of diabetic foot disease.

The POTKU project (Potilas kuljettajan paikalle, Putting the Patient in the Driver's Seat) in Finland aimed to improve patient-centered care by developing personal health and care plans (HCPs) for people with NCDs (2010-2014) and it covered 5 districts in 61 municipalities. The care teams consisted of nurses and doctors who work with the patient to develop the HCP. HCPs included the following domains: (a) my needs (healthrelated problems identified by the patient with the support of a health professional); (b) my goals (what change in health status is strived for; which is the goal of the jointly agreed treatment?); (c) my measures (which health services and measures of the patient planned related to these goals); (d) follow-up and assessment (follow-up appointments to evaluate the implementation of the HCP and its results); (e) medication, diagnoses, and contact person. ${ }^{153}$ The program also monitors the quality and completeness of the HCP and the delivery system. The program resulted in a workable and efficient means to improve patient-centeredness in daily practice. Challenges included non-integrating electronic medical record systems requiring redundant data entry, resistant unit managers, and increased time for nurses and doctors. Results of a patient perception survey (Patient Assessment of Chronic Illness Care ${ }^{154}$ ) indicated that patients who had an HCP perceived the care they received as significantly better on all dimensions than patients who did not have an HCP. Staff also experienced a positive impact on their practice as they tended to see the patient in a more holistic manner and enjoyed partnering with the patient and other staff. Staff also perceived improvements in the quality of chronic illness care with their patients as demonstrated through a survey, the Assessment of Chronic Illness Care. ${ }^{155}$

As a complementary element to multidisciplinary work, standardized training of multidisciplinary teams is critical in implementing and maintaining health care system transformation. The international health care community acknowledges staff training as foundational to execute effective change and successful transformations have used the well-established model of learning collaboratives to achieve transformational goals. The model is particularly useful in training multidisciplinary teams together to support them through performance improvement efforts as they deliver patient care. ${ }^{156}$ Country examples include the national VHA system, Ecuador, Honduras, and Nicaragua. Other integrated health care systems such as New South Wales, Australia focus specifically on multidisciplinary training to achieve stated goals. ${ }^{157}$

The Learning Collaborative Model had significant impacts on improvement in health care worldwide and has been developed by the Institute for Healthcare Improvement (IHI) ${ }^{158}$ which was founded in 1991 and which remains an active leader health care systems improvement today. The model is formally known as the

\footnotetext{
153 http://www.icare4eu.org/pdf/POTKU_Case_report.pdf.

154 The PACIC (Patient Assessment of Chronic Illness Care; MacColl Center for Health are Innovation, Group Health Cooperative, 2004) questionnaire is an international validated instrument that covers several dimensions (from the patient perspective) of what is considered high-quality chronic illness care according to the Chronic Care Model. https://primarycaremeasures.ahrq.gov/care-coordination/downloads/ccatlas/instruments/CC Instrument 10.pdf.

155 The ACIC (Assessment of Chronic Illness Care) questionnaire (MacColl Center for Health Innovation, Group Health Cooperative, 2000) is an international validated instrument that covers several dimensions (from the perspective of health care professionals) of what is considered high-quality chronic illness care according to the Chronic Care Model. https://primarycaremeasures.ahrq.gov/care-coordination/downloads/ccatlas/instruments/CC_Instrument_1.pdf.

156 Institute for Healthcare Improvement 2003.

157 Integrated Care in New South Wales. https://www.health.nsw.gov.au/integratedcare/Pages/default.aspx.

158 Institute for Healthcare Improvement timeline http://www.ihi.org/about/Documents/IHI Timeline 2018.pdf.
} 
Collaborative Model for Achieving Breakthrough Improvement. The approach uses collaborative learning to help "health care organizations make 'breakthrough' improvements in quality while reducing costs. The driving vision behind the Breakthrough Series is that even though sound science exists detailing the approaches and opportunities for health care practice improvement of costs and outcomes, much of this science is not considered or used in daily work, highlighting the gap between what we know and what we do." 159 A Breakthrough Series Collaborative is a short-term (6-15 months) learning system that brings teams together from hospitals/clinics to seek improvement in focused areas such as Turkey's mission to integrate HLCs into the primary care delivery model to improve care for patients with NCDs and promote preventive care. These collaboratives can be conducted regionally using standardized topics and approaches. As noted by the IHI, dramatic results from such collaboratives include reducing waiting times by 50 percent, worker absenteeism by 25 percent, intensive care unit (ICU) costs by 25 percent, and hospitalizations for patients with congestive heart failure by 50 percent. ${ }^{160}$ The IHI also offers a train-the-trainer program in the Breakthrough Series methodology to support health care systems to implement and spread improvement efforts within the cultural context of the organization.

The VHA used the Learning Collaborative model to implement training for a nationwide transformation of its primary care delivery model in $2010 .{ }^{161}$ The Learning Collaborative trained 300 primary care champion teams (4 persons each) from across the country. The champions became the trainers, leaders, and subject matter experts for change locally thereby expanding the reach and spread of the initiative. The training included topical areas deemed critical to ensure standardization, implementation, and performance improvement such as access to care, care coordination and management, population management for chronic illness and prevention care, patient engagement and self-management, team roles, functions and responsibilities, workflow and monitoring, measurement and performance.

The Ministries of Health from Ecuador, Honduras, and Nicaragua launched nationally supported Learning Collaboratives that were conducted in regions to minimize travel costs. The effort was focused on improving maternal care in those countries. Multidisciplinary team training was conducted in person and virtually. The countries partnered with commensurate medical universities to train staff on applying evidence-based medicine interventions. Information on best practices and results were shared on a central website used by all participants. The multidisciplinary teams that participated became champions and implemented training locally. Clinical outcome measures demonstrating high-quality maternity care were significantly improved in each country, prompting continued implementation of the Learning Collaborative model for other services. The most important part of the Learning Collaborative model according to system leaders and clinical staff is the focus on specific clinical processes key for the involvement of staff which is appealing to clinical practitioners. They feel they are learning and truly improving the work they are doing every day, addressing the practical, not just theoretical. ${ }^{162}$

\subsubsection{CURRENT STATE IN TURKEY}

The HLC model recently introduced in Turkey has a good general multidisciplinary framework for service delivery; however, the actual multidisciplinary work in the field is shaping up through local HLC efforts. To

\footnotetext{
159 Institute for Healthcare Improvement 2003.

160 IHI Breakthrough Series College. http://www.ihi.org/education/InPersonTraining/breakthrough-seriescollege/Pages/default.aspx.

161 Bidassie 2014.

162 Institute for Healthcare Improvement 2019.

http://www.ihi.org/resources/Pages/ImprovementStories/UniqueChallengesCreativeSolutions.aspx.
} 
illustrate, Bursa Osmangazi HLC developed a two-week orientation program to successfully adapt new staff to HLC multidisciplinary work. The focus of the orientation program is on the issues specific to Bursa Osmangazi HLC, on behaviors to contribute to multidisciplinary work, and on other technical areas. The orientation program allows the new staff to closely observe and understand the HLC activities, process flow, and operational issues in real time. HLC staff give feedback to the HLC management about the behavioral progress of the new staff toward multidisciplinary work. To address adaptation gaps, the new staff are being given more information or training on relevant topics or they are directed to work in another HLC unit, as appropriate. The Osmangazi HLC team prefers to keep those staff who can adapt to teamwork after the orientation program. One of the motivation sources for the HLC teams is patient satisfaction which also drives HLC staff satisfaction. Bursa Osmangazi HLC has established a case assessment board to regularly convene ${ }^{163}$ the involved staff for the cases in focus to discuss, make decisions, and report about the progress of these cases. When needed, involved FMPs for these patients are also invited to the board meetings.

The interviewees from Hatay HLC indicate that FMPs had a good buy-in in Hatay, and they are happy with referring the patients to the HLC because this procedure is less complicated when compared with hospital referrals. They emphasize that for the buy-in at the secondary level, the legislation on HLCs should be clearly communicated to the secondary care providers. Following a similar line of thought, interviewees from Bursa Osmangazi HLC indicate that primary care (and HLCs) should have the complementary role of follow-up after the specialist treatment.

Field interviews in both Bursa and Hatay reveal that HLC staff would like to be involved primarily on the preventive side of the health care. One reason for this is that the current context necessitates that the FM model operates more on the treatment side and HLC would complement FM on the preventive side. A second reason is the scope of the FM model. While FMCs have their registered population, this setting mostly has an individual patient perspective. But HLCs have a more regional perspective as many of the HLC functions are more population based. A third reason that makes HLCs complementary in primary care is that HLCs have diversified services and equipment that do not currently exist in FMCs.

As has been also indicated in the Strategic Plan 2019-2023, challenges remain about the absence and coordination of specialized workforce in HLCs. ${ }^{164}$ To illustrate, Bursa Osmangazi HLC interviewees indicated they need staff with specialization on obesity, substance addiction, and group therapy.

\subsubsection{RECOMMENDATIONS ON INTEGRATION WITHIN PRIMARY CARE LEVEL}

Increase staff in FMCs. The MoH Strategic Plan emphasizes the importance of increasing the number of the PHC workforce and sets higher targets for 2030. However, as mentioned under Section 3.1.1.2, the health workforce in terms of general practitioners and nurses are well below in international statistics although over the past few years there were improvements in the ratio of nurses. The number of medical graduates in Turkey per 100,000 population has slightly decreased but the number of nursing graduates has significantly increased since 2003 (Table 5).

\footnotetext{
163 As the responsible physician of Osmangazi HLC (Mr. Ahmet Timur) indicates, the board will start its activities soon and plans to meet weekly (Interview on December 12, 2018).

164 This has been indicated as problems and incompatibility within multidisciplinary teams for NCD-related integrated care in the Strategic Plan 2019-2023.
} 
Table 5 Medical and Nursing Graduates per 100,000 Population

\begin{tabular}{|l|l|l|}
\hline Graduates per 100,000 population & $\mathbf{2 0 0 3}$ & $\mathbf{2 0 1 3}$ \\
\hline Turkey Medical Graduates & 6.55 & 6.51 \\
\hline WHO European Region Medical Graduates & 10.12 & 11.37 \\
\hline Turkey Nursing Graduates & 4.49 & 19.77 \\
\hline WHO European Region Nursing Graduates & 31.91 & 40.92 \\
\hline
\end{tabular}

Source: WHO European Health Information Gateway.

This shortage in the staff calls for a more efficient allocation of human resources. Introducing national policy changes that support efforts to increase FM staff in FMCs to more effectively and efficiently manage NCDs would have a profound and positive impact on staff and on patients in receipt of the care. In fact, the MoH intends to increase the FM staff (mostly nurses) in each FMC to two in the future. ${ }^{165}$ The increase in the number of nurses in FMCs will support proactive NCD management. "WHO recommends 2.28 health workers per 1,000 people, though some studies suggest that a still higher ratio may be needed to expand population-based health care services." 166

The $\mathrm{MoH}$ should therefore continue to work with the Higher Education Council to increase and accelerate recruitment and medical education output of the family physician workforce and nursing staff to build the capacity of the system to manage fewer registered patients for each FMP and provide nurses to support NCD management.

Build capacity of physician and support team workforce. The MoH Strategic Plan identifies the need to continue to improve the competencies, motivation, and sustainment of health care staff. Building capacity for physicians and support teams includes but is not limited to identifying and implementing incentives as discussed earlier, increasing career opportunities for service in needed areas, improving working conditions, increasing support for training and role development, improving support for conference attendance and education, and so on. The health workforce planning and policies should address the following activities. ${ }^{167}$

The Right Jobs. Countries need to train sufficient number and proper mix of workers to meet future needs. Countries should endeavor to increase their admission quotas for physicians and nurses known as numerus clausus policies and optimize the scope of practice of different health care providers, removing unnecessary barriers to full scope of practice and promoting innovative and collaborative health care delivery to meet population health needs.

The Right Skills. Countries need to ensure that health care workers acquire and maintain the right skills and competencies to deliver high-quality, team-based, patient-centered care. Education and training programs should be revised in the context of technology and evolving delivery models. Regular recertification of health professionals ensures skills are current.

The Right Places. Countries need to ensure adequate access to health care for the populace. More equitable geographic distribution of health care staff can be achieved through incentives and/or regulations requiring

\footnotetext{
165 Authors' interview with Banu Ekinci, November 7, 2018.

166 Cotlear et al. 2015.

167 OECD 2016.
} 
service in underserved areas, enabling broader scopes of practice for non-physicians, and optimizing use of technology and telemedicine to enhance reach.

Develop standardized staff training and education. Consistent with the objectives in the MoH Strategic Plan 2019-2023 to improve the competence and skills of the health care staff, it is recommended that staff of the HLCs, FMCs, and secondary care sites, with whom they are involved, receive standardized education and training to support the implementation of integrated care as outlined by the Minister of Health and Turkey's government. The $\mathrm{MoH}$ can rely on the training/learning model that has been developed by the IHI, known as the Collaborative Model for Achieving Breakthrough Improvement, as discussed earlier under Section 3.3.1.1. The approach uses collaborative learning to help health care organizations make 'breakthrough' improvements in quality while reducing costs. As leaders and staff need to fully understand and then engage in learning to integrate principles, concepts, and expectations to implement in an effective, efficient, and consistent manner, this training can be incorporated into MoH's general on-the-job training curricula. Team-based experiential learning that is consistent with leadership expectations, mission, vision, and values is foundational to system and culture change. The training program is expected to present a road map for leaders and staff to emulate and implement, share ideas and best practices, and immerse themselves in improving the health care delivery system in which they work. Topical areas of focus should include basic and advanced concepts of integration such as vision, mission, goals and objectives, expectations of performance and associated metrics, standardized teamwork to optimize efficiencies and effectiveness, defining specific roles and functions for each team member, outlining clinical and administrative communication/planning approaches and expectations, addressing expectations for improving care management and coordination among the delivery systems, focusing on techniques and methods to better manage NCDs and preventive care, and the principles in patient engagement and patient-centered care.

\subsubsection{CARE COORDINATION}

\subsubsection{OVERVIEW AND COUNTRY EXAMPLES}

Care coordination is a foundational element of an effective and efficient integrated care delivery system. "Care coordination is a deliberate organization of patient care activities between two or more participants (including the patient) involved in a patient's care to facilitate the appropriate delivery of health care services. Care must be organized and involves the marshaling of personnel and other resources needed to carry out all required patient care activities and is often managed by the exchange of information among participants responsible for different aspects of care." 168 Effective care coordination requires building relationships between PHC teams, and key specialist groups, hospitals and community service agencies. Tools such as protocols to support successful referrals and transitions, and systems to support information transfer should be developed to aid efforts of care coordination. 169

Additionally, one of the tenets of cost-effective health care system management is shifting low complexity care out of the hospital system into the PHC system. ${ }^{170}$ This shift also facilitates a more effective care coordination. Visits to the ED and unnecessary inpatient admissions, where the care could have been more appropriately

\footnotetext{
168 Heslop, Power, and Cranwell 2014; Schultz et al. 2013.

169 CARE COORDINATION Reducing Care Fragmentation in Primary Care http://www.safetynetmedicalhome.org/sites/default/files/Executive-Summary-Care-Coordination.pdf.
}

170 Advisory Board 2014; Berchet and Nader 2016; Driscoll 2017; O’Malley 2012; Quackenbush, Shenkel, and Schatz 2004; Tranberg et al. 2018. 
delivered in a PHC environment, are costly and can have significant negative impacts on the quality of care. Health care systems seeking solutions to low complexity ED and inpatient services overcrowding understand that access to PHC services is a crucial factor in addressing patient needs. ${ }^{171}$ Partnering and coordination practices of secondary and primary care, information sharing, and feedback mechanisms for each patient therefore constitute an integral part of efforts to integrate care.

Provision of primary care for after hours is another method pursued to shift the care workload from emergency care toward primary care. One study noted that hospitalization due to chronic disease for ACSCs was less likely when patients had access to PHC after hours. ${ }^{172}$ Though estimates vary, one study calculated that 13.7 to 27 percent of visits to EDs could have been taken care of in lower complexity outpatient settings, saving roughly US $\$ 4$ billion each year. ${ }^{173}$ Expanding access to primary care after hours has been shown to reduce barriers to care, improve care coordination and continuity, reduce treatment delays, and improve trust and reliance on the PHC system, thereby reducing ED visits and other non-primary care services. ${ }^{174}$

For Germany's Gesundes Kinzigtal scheme, promotion of care coordination is key to achieve the triple aim of improving the health of the Kinzigtal population, improving the patient experience, and reducing the per capita costs of care. ${ }^{175}$ Multimorbid patients are the first cohort targeted for an integrated care plan. To improve patient compliance and health outcomes, the model encourages information sharing, consultation among care providers, and care coordination. To illustrate, participant providers consult with clinical pharmacologists six times a year to improve the medication regimen of multimorbid patients. The physician submits a case report to the clinical pharmacologist who evaluates the medications and gives feedback to the physician in terms of scores on the physician's prescription behavior. The pharmacologist provides additional feedback on how to improve the medication schedule. ${ }^{176}$ Physicians can also work with social workers to improve the care provision, which requires adaptation to the comprehensive needs of the multimorbidity. Partnerships with hospitals include the post-discharge care coordination process to reduce readmission rates and to improve continuous care. Defined procedures are part of the service contract between the hospitals and the Gesundes Kinzigtal scheme. Care pathways are developed and agreed upon jointly by hospitals and other care providers. ${ }^{177}$

To provide feedback on the implemented coordination practices, a committee comprising physicians, therapists, psychologists, program managers, and Gesundes Kinzigtal management conduct a program-level cost-benefit analysis every 18 months and gives feedback to program administrators to provide guidance on the adjustments to be made and new features to be introduced to the program. ${ }^{178}$

\footnotetext{
171 Berchet and Nader 2016; Hempel et al. 2018; O’Malley 2013; https://www.healthcaredenmark.dk/media/1625194/HCD-Telehealth-white-paper-v1-single-0318.pdf.

172 Augustine et al. 2016.

173 VA re Weinick, Burns, and Mehrotra 2010.

174 Chaiyachati et al. 2014.

175 https://www.selfie2020.eu/wp-content/uploads/2016/12/SELFIE WP2_Germany_Final-thick-descriptions.pdf. 176 http://vbhcglobalassessment.eiu.com/an-integrated-approach-to-value-based-healthcare-germanys-gesundeskinzigtal/.

177 https://www.selfie2020.eu/wp-content/uploads/2016/12/SELFIE_WP2_Germany_Final-thick-descriptions.pdf. $178 \mathrm{http}: / /$ vbhcglobalassessment.eiu.com/an-integrated-approach-to-value-based-healthcare-germanys-gesundeskinzigtal/.
} 
As a second example, the Zio model in the Netherlands assigns a central role to nurses and support staff through delegating tasks traditionally under the responsibility of physicians. To illustrate, two types of nurses operate for diabetic care model. While practice nurses support the primary care, diabetic nurse specialists provide advanced care and act as consultants to general practitioners. Patients with low and medium intensity care are under the responsibility of nurses, and specialists such as endocrinologists assume responsibility for patients in need of high-intensity care. Redistribution of the health workforce this way reduces the outpatient workload of specialists and presents a good alternative to standard general practitioner care. The primary care units coordinate care across multiple providers and are responsible for regular patient follow-up and case management. 179

\subsubsection{CURRENT STATE IN TURKEY}

Concerning care delivery and coordination, one of the main bottlenecks of the Turkish health system is the high utilization and workload of emergency services. The MoH Strategic Plan 2019-2023 highlights the intention to address the issue of reducing the burden of emergency room (ER) services under Goal 3 (to ensure effective and high-quality care delivery) by bringing more effectiveness and productivity for outpatient and emergency care and mentions the need to increase and expand triage control and audit as well as to increase awareness on proper use of emergency services. This need is also supported with data from the MoH GDHIS which suggest that throughout Turkey there is high utilization of ER services after business hours (particularly between 1600 and 2400 hours and during weekends). Data also suggest that the majority of these cases have a triage category yellow (having a share of 64 percent of total ER visits) or green (having a share of 22 percent of total ER visits), implying that many of these cases can actually be addressed at the primary care level 180 (Figures 19, 20, and 21 and Annex 1). This calls for arrangements to reduce emergency care workloads.

\section{Figure 19}

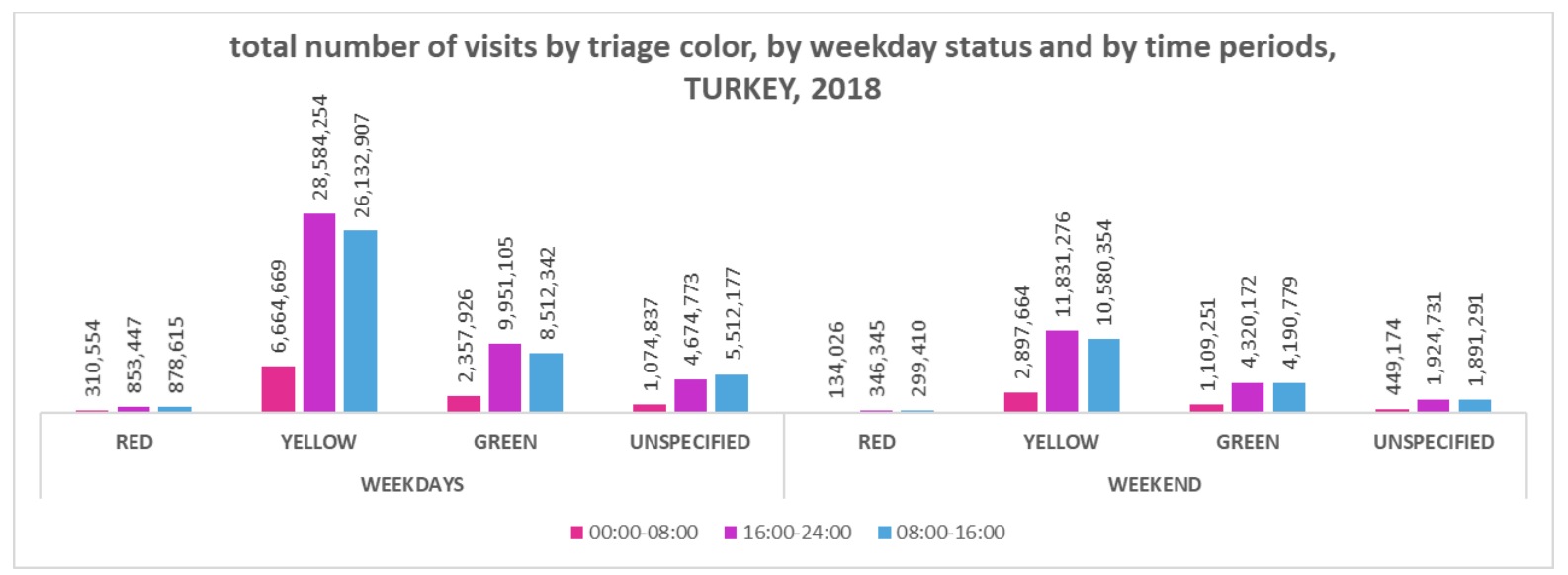

\footnotetext{
${ }^{179}$ Hubertus et al. 2017.

180 Detailed data analysis for ER visits in the six biggest cities of Turkey are given in Annex 1.

The green category for triage indicates outpatient cases that have a stable general condition with simple health problems. The yellow category for triage indicates cases that might carry life threatening risks and risks of significant morbidity as well as cases that have a potential to be severe with moderate to prolonged symptoms. The red category for triage indicates cases with concrete life threats requiring urgent evaluation and treatment (Ministry of Health of Turkey, Emergency Department Emergency Procedure, 2013, https://studylibtr.com/doc/769891/t.c.-sa\%C4\%9Flikbakanli\%C4\%9Fi-t\%C3\%BCrki\%CC\%87ye-kamu-hastaneleri\%CC\%87-kurumu)
} 
Figure 20

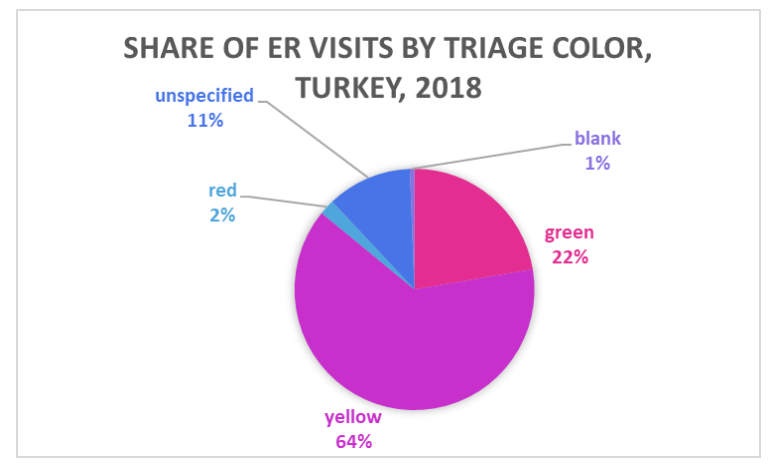

Figure 21

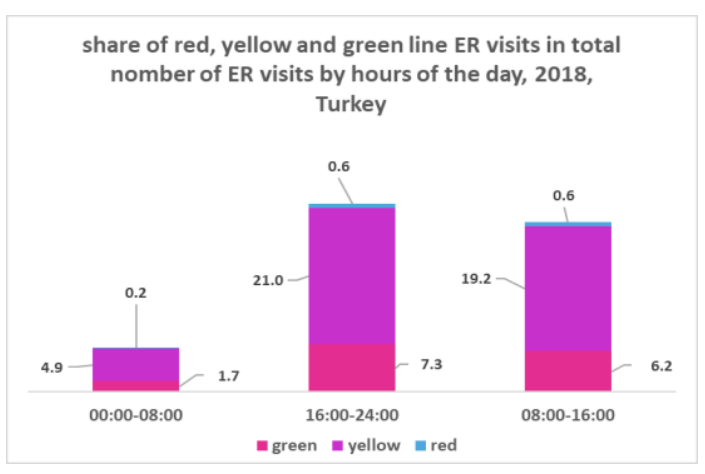

While integrated care primarily focuses on the 'way to do things instead of instruments or devices'181 and prioritizes activity flow and processes, currently, no formal care coordination and referral process between primary and secondary care and primary care structures (FMCs and HLCs) has been defined in Turkish health system. ${ }^{182}$ Information systems used at different levels of care are not integrated. ${ }^{183}$ While there is no legal policy preventing any HLC staff from documenting their activities, HLCs do not have clinical documentation or performance tracking technology systems for multidisciplinary staff. In Bursa Osmangazi HLC, the correspondence and communication between HLCs and FMCs are through the electronic document management system (EBYS) but this is not a database; it is a safe document correspondence tool.

In the absence of clear definition of roles and responsibilities, there is no clear role sharing ${ }^{184}$ among care provider teams and there is no national standard for training staff in FMC and HLC integration. ${ }^{185}$ Clearly defining the workflow and processes and training $\mathrm{MoH}$ implementing staff on those processes are imperative to orient these staff for behavioral change. To this end, the MoH Department of Public Health Services and Training has recently started working on and updating the workflow diagrams and HLC guidelines which detail the roles and responsibilities of health care staff.

HLCs are managed by a responsible physician appointed by the Provincial Health Directorate. The responsible physician is in charge of the allocation and reallocation of staff into the HLC service units, the proper conduct of work and procedures in the HLC, and the HLC's official correspondence with the MoH. A registration/information desk is formed at the HLC entrance where medical secretary and other appropriate personnel are responsible for registering the patients in the registration system and referring them to the related HLC unit. HLCs also offer the transportation services for patients referred to HLCs by FMCs.

\footnotetext{
181 European Union 2017.

182 The new Strategic Plan acknowledges this by highlighting that job descriptions and job boundaries for FMPs and hospital services are unclear.

183 The new Strategic Plan also highlights this gap with an additional emphasis that HIS is not effectively used especially for NCD management.

184 In VHA primary care teams, everyone has a specific role and care is distributed. The HLC can help offload the responsibility of the physician.

185 While the MoH provides HLC training for the HLC staff, this training lacks features addressing individualized care such as different patient communication models according to patient behavior or preferences, as has been indicated in the Strategic Plan 2019-2023.
} 
Transportation is provided by vehicles with easy boarding at least five times daily on the defined routes and at the defined times. ${ }^{186}$

Currently, the HLC case coordinator is supposed to manage only the administrative process between providers (to manage appointments between FMCs, HLCs, and hospitals) and not the medical process. Therefore, the HLC case coordinator does not necessarily have to be a nurse. The role of the case coordinator is usually defined under the medical secretary where the roles and responsibilities are to carry out correspondence and communication for the continuity of care; manage appointments and patient registration; keep patient administrative and medical data and convert all medical data into medical documents; file, archive, and retrieve all patient data; carry out clinical coding; compile statistical data and prepare reports for the upper management; and manage patient discharge work and communications. ${ }^{187}$

While the current position of a case coordinator mostly assumes an administrative role, the responsible physician from Bursa Osmangazi HLC indicates that the case coordinator should be able to make a holistic patient assessment including his/her need for a referral to higher levels of care, his/her status, and should be able to take initiative. ${ }^{188}$ Concerning NCDs, the follow-up data collected by the case coordinator can then be analyzed and shared with the FMC for further coordination and patient care. Interviewees from Hatay HLC indicate the lack of legislative background which sets forth the relation and coordination of HLCs with FMCs. They mention that the $\mathrm{MoH}$ guidelines exist, but emphasize that these guidelines are generic and usually need good interpretation for actual implementation. To illustrate, they give the example of the case coordinator selection. The guidelines were not clear enough on the selection of the care coordinator; therefore, the HLC staff made their own interpretation and have selected the case coordinator to be a presentable and welcoming person for the patients.

Despite the lack of central guidance on care coordination, Bursa and Hatay were successful in developing their own good local coordination practices that HLCs pursue in the field. One example is cancer screening. In Bursa Osmangazi HLC, people are called individually for cancer screening or they are contacted through their family physician. They are then referred to secondary care in Bursa if their screening results are positive or suspicious. This referral is done manually with a paper given to the patient. With this 'referral paper', the patient can avail the same-day service at the secondary level. A second example is peculiar to Hatay province. Because of the demographics and thalassemia being a hereditary disease, its prevalence and incidence is high in Hatay. All couples to be married are to be mandatorily tested for thalassemia. These tests are conducted in Hatay HLC and those with positive and suspicious test results are referred to secondary care. These two good practices can be the role model to scale up care coordination of HLCs with secondary care.

As a small-scale support to care coordination efforts, the General Directorate of Public Health trained HLC staff acting as case coordinators in the 18 HLCs within the scope of the project jointly conducted by the World Bank and the General Directorate of Public Health. The training was on the use of data and data entry in the online registration form designed for the HLC information desks, as patients' initial contact in the center. Additionally, the General Directorate sent the list of these trained staff to FMPs so that they can contact these staff in referring patients from FMCs to HLCs. Although the data entry practice is not uniform among the participating HLCs, currently data are collected through this form.

\footnotetext{
186 MoH 2018.

187 https://dosyaism.saglik.gov.tr/Eklenti/12541,20140522-29007-sag-meslek-mensuplari-ile-sag-hizde-cal-digermeslek-mensuplarinin-is-ve-gorev-tanimlarina-dair-yonetmelikpdf.pdf?0.
}

188 The position mostly looks like a case manager. 
Another local pilot initiative for coordination by the General Directorate of Public Health is in the province of Kırklareli, where a brief paper-based referral form has been designed, and FMPs are asked to use this form for their referrals to HLCs. Data from these forms are later shared with the Chronic Disease and Elderly Care Department.

One additional area where there can be care coordination between primary and secondary levels is obesity. Obesity Treatment Centers ${ }^{189}$ for the morbidly obese patients ${ }^{190}$ have been established as part of the secondary care system in Turkey where patients are identified and referred by FMCs and hospitals. The program pursued in these centers is a yearlong robust scheme and has a comprehensive set of modules and interventions with a multidisciplinary team that works with other ministries and municipalities. Once a patient is in the center, Obesity Treatment Centers 'own' the patient (including all diseases) for a year of their intervention and treatment. Record keeping in these centers is currently manual (on paper) and is not integrated with primary care databases (FMC, HLC, and NCD) and hospital databases (hospital information system [Hastane Bilgi Yönetim Sistemi, HBYS]). Obesity Treatment Centers have developed their treatment algorithms on paper and have put them into practice. However, because there is currently no national technology platform (database) in these centers, they need 'off the shelf' or self-developed software to transfer these algorithms and data into the digital medium. To retain details about the medical examination of obese patients, the interviewee ${ }^{191}$ for the Obesity Treatment Centers indicated that it is important to include the obesity module into the general referral system because HBYS currently maintains data on the patient visits but maintains no data or documents the care for the obese patients.

Maximizing communications. In the absence of mandatory referrals between and within the same levels of care, ${ }^{192}$ the need to create awareness for both the patient and providers concerning the use of PHC becomes more important. Local teams have a good understanding of this need and they actively promote HLCs and their services. In Bursa, to convince specialist physicians on HLCs, HLC staff call and explain the HLC system and its necessity and complementarity for the FM model. They also visit FMPs and promote their activities. The interviewees highlight that if patients convey a positive feedback to FMCs on HLC services, this is a strong indication that the HLCs are preferred and there is satisfaction for HLC services. In Hatay, the case coordinator, who is a medical secretary, makes scheduled visits to rural areas and villages by a vehicle and brings patients to the HLC (for cancer screening and thalassemia). When the patients are in the HLC, they are also informed about all the HLC services. Additionally, HLC services are promoted in schools and in FMCs in Hatay.

\subsubsection{RECOMMENDATIONS ON INTEGRATION WITHIN PRIMARY CARE LEVEL}

Increase/enhance HLC visibility and buy-in on both provider and patient side. The services of HLCs will gain recognition and sustainability with the increased awareness and providers' and patients' acceptance of HLCs. As the local good practices reveal, on the provider side, there is a need to convince FMCs on HLC functions and how they complement primary care. If HLC and FMC staff have their roles clearly defined for coordination, this would increase the FMP ownership of HLC services. As a non-monetary incentive, the head of GD Chronic Diseases and Elderly Care highlights that formal recognition of family physicians highly referring to HLCs can

\footnotetext{
189 Currently there are 34 Obesity Treatment Centers, 29 new ones are planned within 100 days as of November 1, 2018, and in all provinces in the future.

190 Target population is people with body mass index $>35$.

191 Ms. Hatice Kınık.

192 From a health system resources point of view, the head of GD Chronic Diseases indicates that mandatory referral between FMCs and HLCs can be possible when the number of HLCs reaches more than 1,000.
} 
be useful. She also indicates that with the new structure, coordination should be from FMCs to HLCs and from there to hospitals. This flow needs to be well communicated to the FMPs.

On the patient side, as the Bursa Provincial Health Director indicates, it is important to address NCD patients at the primary care level (within FMCs and HLCs) and important to genuinely listen to them. He emphasizes that the majority of the multiple provider visits occur since the patient is not able to find the quality of care he/she is looking for. Genuinely listening to the patients, understanding their problems, and communicating with them in their own language at the first point of contact at the primary level would reduce the visits to ERs and hospitals.

Establish formal practice agreements. The MoH should consider establishing formal practice agreements, also called service or care coordination agreements. These agreements are non-fiscal in nature supporting collaborative ways of working and should be mutually negotiated between HLCs and FMCs. This practice tangibly supports the concept of integrated care and promotes the work of the collective team in managing NCD patients. The content of formal practice agreements usually includes expectations for both sides about consultation, referrals, and patient management. Agreements are negotiated and list the agreed expectations for what the HLCs and FMCs will provide. Examples of topics usually included are the process to prepare patients for the interface between HLCs and FMCs, patient's responsibilities, lines of communication between the practices, information provided to each actor within the process, type and timing of requested services, response and follow-up for providing information and time frames, and specific points of contact for direct communication and needs.

Practice agreements should focus on which discipline is responsible for coordinating care and what that means in terms of integrating the care between the FMC and the HLC. For example, the role, function, and responsibilities of the case coordinator should be addressed in detail, describing how patients are identified and how the coordination of the patient care between FMCs and HLCs is made and supported.

While there is currently no coordination between Obesity Treatment Centers and HLCs, the interviews revealed that the use of HLC sports centers by the patients of the Obesity Treatment Centers can be an area of cooperation. As needed, Obesity Treatment Centers currently advise patients to go to the sports center, but they do not have such facilities under their premises. With proper coordination and possible practice agreements with HLCs, they can refer their patients to HLC's sports centers. Such practice agreements between HLCs and the Obesity Treatment Centers will strengthen the relationships between these two facilities.

Offer after-business-hours access to PHC services. Thoughtful consideration should be given to the concept of increased access to PHC services after normal business hours to reduce unnecessary visits to EDs. Access can be accomplished in several different ways through rescheduling FMCs' operating hours a few hours past normal business closing time, opening FMCs on a weekend morning, or using available technology systems (telemedicine/call centers, and so on) to triage patient needs and complaints to the appropriate level of care and follow-up. Other ideas to improve after-hours access in primary care health services would be to (a) authorize overtime pay; (b) offer flexible scheduling so if staff work a day or half day on a weekend, they are given commensurate time off during the work week; or (c) pay a premium rate for staff willing to work on weekends. A system-level change would be to charge patients for green line services in the ED which may shift the volume to PHC services. Currently, available nationwide data can provide important information that the $\mathrm{MoH}$ would need to identify areas and populations where establishing increased access to lower cost delivery systems for after business hours would be of benefit.

Specifically define roles of FMC practice nurse and HLC coordinator for care coordination, NCD and transition management. The $\mathrm{MoH}$ and subject matter experts might consider redefining the roles of practice 
nurses and HLC coordinators to achieve a more standardized approach to manage care coordination, NCDs, and prevention services at the primary care level. As mentioned, care coordination is an essential element of integrated care among FMC, HLC, and secondary health care systems/providers. The PHC system (FMC and HLC) should be accountable and responsible for coordinating the patients' needs and preferences for health services and information sharing across people, functions, and sites, as well as organizing patient care activities between two or more participants (including the patient) involved in a patient's care process to facilitate appropriate delivery of health care services at the right time and place.

As the roles, functions, and workflow of nursing staff within FMCs and care coordinators in HLCs are revised, these staff will need standardized training, education, and support to learn and implement new job requirements. Clinical protocols, competencies, and functional pathways describing the roles of each primary care team member (FMC and HLC) have been used internationally to effectively and efficiently provide safe care. The following discussion explores how the roles and responsibilities of nurses and care coordinators can be shaped in the Turkish context:

- Nurses. Good country examples for the roles and functions of PHC practice nurses that can be incorporated into the FM model and that can support the concept of 'top of the license' functioning, shifting the work from physician to other qualified staff, including the following:

- Working with physician and HLC staff to identify specific populations of registered patients to manage high-risk patients (lacking an effective primary care partnership, as evidenced by ER visits that outnumber primary care visits, poor specific NCD clinical indexes, multiple hospital visits in the ED or inpatient for ACSCs or for complications, and multiple uncoordinated system contacts).

- Managing identified specific populations by conducting in-depth and ongoing review of their clinical status, by providing counseling/intervention and training to patients and their families on clinical needs consistent with evidence-based CPGs/patient pathways. This work can be conducted virtually (telephone, text apps, e-mail, and so on) or in person. The nurse would require an appointment grid so appointments can be scheduled on the same day or prescheduled.

- Initiating appropriate consultations to other FMC staff or HLC ancillary services as defined by new scope of practice, competency, or pathway.

- Triaging registered patients who call or present for non-emergency acute care needs and recommending care based on evidence-based guidelines. For example, patients experiencing symptoms of a common cold (as defined by medical evidence literature) could be advised to stay at home or go home, drink fluids, take over-the-counter medications, and call the practice nurse if symptoms worsen within a specified time frame. These patients would not require a visit with the physician. The nurse would be responsible for documenting the care provided consistent with the patient's subjective presenting symptoms, nursing assessment, and care plan/intervention and follow-up.

- Conducting, collaborating, and partnering with other FMC, HLC, and program services for preventive care and surveillance of registered population based on evidence-based guidelines (for example, colorectal cancer screening, immunizations, breast and cervical cancer, childhood development, health education and counselling on healthy behaviors, smoking, depression, alcohol consumption, and behaviors,). 
- Supporting NCD management and care activities with medication reviews and medication reconciliation, ${ }^{193}$ focusing on specific tasks of care/education required by the chronic disease, ${ }^{194}$ self-management education, reviewing labs/tests pertinent to NCDs to assess clinical status, and collaborating with physician to determine next steps in care regimen. ${ }^{195}$

- Providing timely follow-up care and management for patients following discharge from ED or inpatient services to shift low complexity care out of the hospital and reduce avoidable ED or hospitalizations. This task requires partnering with local hospital systems, often called as service agreements or care coordination agreements, to foster care coordination. For example, contents may include prompt notification of hospital admissions, communication with the clinic before discharge, collaboration between hospital/specialty support staff and practice support staff post-discharge, prompt FMC access to a comprehensive discharge summary, and a timely post-discharge clinic visit or telephone call by primary care. ${ }^{196}$

- Communicating test results and care plans to patients and families. Enabling a practice nurse versus a physician to tell patients about their test results over the telephone or in person will improve clinic efficiency and the patient experience. Other national health systems, such as the VHA, have developed policies and guidelines supporting this concept. ${ }^{197}$ The MoH should consider developing a policy that promotes this practice in FMCs and HLCs.

- Care coordinators. The role encompasses coordinating the care within the practice, and between settings, including community sites, labs, specialists, and hospitals. The literature describes different health care roles and disciplines that perform as care coordinators including medical assistants, social workers, nurses, community health workers, and so on. The care coordination workforce manage care for patients with multiple chronic conditions requiring a multiskilled approach to support complex care and social support needs as they are typically high users of health, community, and social services. ${ }^{198}$ Whether care coordinators are clinical or nonclinical staff depends on the function required. Most systems reviewed, however, opted for a clinical staff who has the knowledge, skills, and abilities to assess and manage health care needs as a means for effective and efficient care delivery. As described by the medical director in the Bursa HLC, the care coordinator "should be able make a holistic patient assessment including his/her need for a referral to higher levels of care, the patient status and be able to take initiative." Although the MoH has defined the role and functions as

\footnotetext{
193 Medication reconciliation is the process of creating the most accurate list possible of all medications a patient is taking-including drug name, dosage, frequency, and route-and comparing that list against the physician's admission, transfer, and/or discharge orders, with the goal of providing correct medications to the patient at all transition points within the hospital.

http://www.ihi.org/Topics/ADEsMedicationReconciliation/Pages/default.aspx.

194 Diabetes Teaching Aids. US Department of Veteran Affairs/Department of Defense Clinical Practice Guidelines. https://www.healthquality.va.gov/guidelines/CD/diabetes/CPG DTSMS01cHowToUseTheFlipChart.pdf. https://www.healthquality.va.gov/guidelines/CD/diabetes/DiabetesTeachingChecklist.pdf.

195 WHO 2010b; US Department of Veteran Affairs/Department of Defense Clinical Practice Guidelines. https://www.healthquality.va.gov/.

196 Carrier, Dowling, and Pham 2012.

197 VHA Directive 1088. Communicating Test Results. https://www.va.gov/vhapublications/ViewPublication.asp?pub ID=3148. 198 Heslop, Power, and Cranwell 2014.
} 
administrative versus clinical, the HLC leader contacted finds more value in the role provided by a clinical staff. Typical functions/tasks of the care coordinator found in the literature include the following:

- Administrative. Referral and transition management are generally administrative tasks including providing information and logistical help to referred patients, ensuring timely and effective transfer of patient information, and tracking referrals and transitions to identify and potentially remedy glitches. ${ }^{199}$

$\circ$ Clinical

- Follow-up - monitoring and assessing patients at regular intervals.

- Self-management support - providing information and counseling to help patients set goals and develop action plans to more effectively self-manage their health and illness.

- Medication management - performing medication reconciliation; evaluating medication adherence, effectiveness, and toxicity; and recommending or making guideline directed changes in regimen.

- Emotional support - monitoring the patient's psychosocial state and recommending appropriate mental health or supportive interventions when necessary.

- Care coordination - helping coordinate care when patients need services from other providers, institutions, or agencies.

\subsubsection{EVIDENCE-BASED PATHWAYS AND PROTOCOLS}

\subsubsection{OVERVIEW AND COUNTRY EXAMPLES}

Evidence-based health care and practice has increasingly become the norm. Factors contributing to the rise in evidence-based health care include "cost pressures, technology advances, increase in management-led decision making, changing public expectations, value for money movement, availability of information, political consensus, non-clinicians with the authority to question effectiveness, international consensus, professional accountability and changing demographic profile."200 The use of evidence-based pathways and protocols is now widely considered a value-added approach to improve patient health care outcomes and efficiently use multidisciplinary teams to deliver standardized care and work processes. ${ }^{201}$ Smartcare Integrated Pathways piloted in 10 regions in Europe ${ }^{202}$ and HealthPathways implemented in Canterbury, New Zealand, ${ }^{203}$ are

\footnotetext{
199 Referral Tracking Guide. American College of Physicians.

http://www.improvingchroniccare.org/downloads/3_referral_tracking_guide.pdf.

Referral Coordinator Job Description. American College of Physicians.

http://www.improvingchroniccare.org/downloads/4 referral coordinator job description.pdf.

200 Hamer an Collinson 2005.

201 Greenhalgh, Howick, and Maskrey 2014.

202 SMARTCARE: Delivering integrated eCare. http://www.pilotsmartcare.eu/home.html.

203 The King's Fund 2017.
} 
examples of effective use of evidenced-based approaches that enable health and social care professionals to deliver the best possible care to patients.

Integrated care pathways and evidence-based medicine protocols can be highly effective in supporting individuals to receive the relevant clinical care on time. Ineffective care reflects nonadherence either related to a lack of knowledge or compliance by point-of-care professionals. Providers in Kenya diagnosed patient vignettes correctly only 16 percent of the time, similar gaps in application and understanding of knowledge have been found in the United States (67 percent) and other countries. There are significant data in the literature that the use of evidence-based medicine, guidelines, and protocols results in better health outcomes. ${ }^{204}$ Setting standards, with evidence-based protocols, can establish consistency in delivery of highquality care across diverse health systems. Guidelines and protocols can also improve staff knowledge and support redesign of workflow within a clinic.

Germany, the Netherlands, Basque Country, and the United States are good practices examples for care pathways.

In Germany, being aware of the German health system's drawback on the absence of after-discharge follow-up and care coordination, Gesundes Kinzigtal aims to introduce better follow-up coordination between hospitals and other providers through jointly developed care pathways. While an enrolled patient can choose his/her 'doctor of trust', the physician's assistant will later take charge of a continuous follow-up care. By improving the quality of follow-up care, the Gesundes Kinzigtal scheme aims to prevent unnecessary and costly readmissions. ${ }^{205}$

In the Netherlands, type 2 diabetes care is provided based on the national evidence-based care standards which set forth the norms for quality care concerning NCDs. Through the negotiations among care groups, health care professionals, and health insurers, care standards are reflected in the specific care protocols and care is delivered and reimbursed based on these protocols. ${ }^{206}$

In Basque Country, as transformation strategy suggests both context-specific and local solutions, a number of integrated care models are being implemented. One of the models focuses on process integration that requires the development of integrated clinical pathways for chronic diseases such as COPD, heart failure, and diabetes. These clinical pathways are jointly implemented by primary care and specialized care providers. ${ }^{207}$

The VHA ${ }^{208}$ has integrated $\mathrm{CPGs}^{209}$ for many common illnesses and NCDs typically treated in primary care into their point-of-care delivery system through the electronic medical record. The electronic patient record contains all clinical information necessary to deliver care services including problem lists with active and nonactive diagnoses, all medications prescribed by the VHA and non-VHA providers, all lab and radiology diagnostic orders and results, all consults/referrals and results, all progress notes and care delivered for all inpatient and outpatient services and evidenced-based protocols and guidelines tailored to each patient. The VHA clinical staff use CPGs to design the workflow and define the roles/functions of each multidisciplinary staff

\footnotetext{
${ }^{204}$ Hamer an Collinson 2005.

205 Hildebrandt et al. 2010.

206 https://bmcfampract.biomedcentral.com/articles/10.1186/s12875-015-0320-z.

207 Solinis et al. 2013.

208 The VHA is the largest vertically and horizontally integrated care network in the United States, providing care at 1,250 health care facilities, including 172 medical centers and 1,069 outpatient sites of care of varying complexity (VHA outpatient clinics), serving 9 million enrolled veterans each year. https://www.va.gov/health/index.asp.

209 Veterans Health Administration Clinical Practice Guidelines. https://www.healthquality.va.gov/.
} 
member on the team. Automated prompts remind staff when patients are due for annual prevention screening or chronic care management. For example, the CPG for diabetes requires the patient to have a foot exam conducted and documented at every visit. Nursing staff are trained to complete and document the findings of the foot exam which are then reviewed by the primary care provider for determination of whether a foot is 'at risk' and requires a referral to a specialist.

Before the VHA had a robust electronic medical record and relied on a paper chart, knowledge of and adherence to CPGs were based on a checklist kept at the front of the paper chart. Each common ambulatory diagnosis (for example, diabetes, COPD, and so on) had a checklist of requirements on which clinical staff indicated they have completed (checking a box, dating, and signing) and documented the care in the progress notes. This type of manual system supported a practical and pragmatic approach to evidenced-based care delivery. Adherence to the guidelines was monitored and the objective results of lab data and medications were compiled to get a complete picture of health outcomes. These results were shared routinely with teams at the primary care centers to address improvements in care delivery.

\subsubsection{CURRENT STATE IN TURKEY}

There is a high-level intention to introduce and implement care pathways and protocols as well as to develop clinical quality standards for evidence-based practice. This demonstrates positive and intentional commitment in support of integrated care and NCD management. Increased use of these protocols and standards would ensure uniform implementation throughout the country. The MoH Strategic Plan 2019-2023 highlights the intention to strengthen the PHC and MoH plans to establish a regular individual-based NCD follow-up system which would incorporate comprehensive patient information and design all PHC end-to-end processes for NCDs. The Strategic Plan indicates that the processes should be standard and aim to improve the patient experience. Case-based clinical treatment protocols, decision trees, service points, actors to assume the roles, and service delivery flow diagrams will be the features to be included in the process design. Clinical and administrative processes also have to be thoroughly reflected to the HIS and there should be perfect information flow between stakeholders.

As a concrete example at the central level, MoH General Directorate of Public Health (Chronic Diseases and Elderly Care Department) has clearly identified the role of the primary care providers and developed patient pathways for the prioritized NCDs: HT, diabetes, obesity, and CVD risk assessment (Annex 2 indicates the roles of FMCs and HLCs as well as two of these pathways: HT and CVD risk assessment). These pathways have been designed end to end (therefore they include the entire process) and are expected to be used by the end of 2019, when UHTS and its NCD sub-module become operational. To support this effort, population awareness activities are carried out for these four conditions, health worker training modules for the interventions on these four conditions have been developed, and health workers have been trained on these interventions. UHTS will allow to track the compliance to screening and follow-up for both the patient and the provider and will keep data on clinical details of these interventions (for example, for HT, data on blood pressure levels of the patient will be systematically collected on disease management platform (HYP). ${ }^{210}$

210 Feedback from the Head of Chronic Diseases and Elderly Care Department, December 3,2018. 


\section{Table 6}

\begin{tabular}{|c|c|c|}
\hline $\begin{array}{l}\text { Activities for which workflow } \\
\text { algorithms developed }\end{array}$ & $\begin{array}{l}\text { Positions for which job } \\
\text { definitions drafted }\end{array}$ & Forms developed for data collection \\
\hline $\begin{array}{l}\text { Patient registration (individual visit, } \\
\text { referral from the FMC or other health } \\
\text { institution) }\end{array}$ & $\begin{array}{l}\text { - } \quad \text { Data entry staff } \\
\text { - Consulting nurse for the } \\
\text { information/reception desk } \\
\text { - General practitioner }\end{array}$ & $\begin{array}{l}\text { - Osmangazi HLC medical } \\
\text { examination form }\end{array}$ \\
\hline $\begin{array}{l}\text { Referral of incoming patient to the } \\
\text { healthy nutrition and obesity unit } \\
\text { within HLC or to secondary care }\end{array}$ & $\begin{array}{l}\text { - } \\
\text { - } \quad \text { Diensulting nurse } \\
\end{array}$ & $\begin{array}{l}\text { - Osmangazi HLC patient consent } \\
\text { form to share patient data with the } \\
\text { FMP } \\
\text { - Patient data form to be shared with } \\
\text { the FMP }\end{array}$ \\
\hline $\begin{array}{l}\text { Screening for colorectal, breast, and } \\
\text { cervical cancer and referral to } \\
\text { secondary care as required }\end{array}$ & & $\begin{array}{l}\text { - Screening results form - patient list } \\
\text { with cancer screening test results }\end{array}$ \\
\hline Psycho-social counseling & & $\begin{array}{l}\text { - } \\
\text { Osmangazi HLC preliminary patient } \\
\text { assessment form } \\
\text { - } \quad \text { Action plan for the patient } \\
\text { - } \quad \begin{array}{l}\text { Form for referral to and } \\
\text { collaboration with other health } \\
\text { institutions }\end{array} \\
\text { - } \quad \text { Interview form for the patient and } \\
\text { his/her family } \\
\text { Osmangazi HLC patient consent } \\
\text { form to protest data privacy and if } \\
\text { needed share patient data with } \\
\text { other professionals. }\end{array}$ \\
\hline
\end{tabular}

Because pathways and protocols are still mostly at the theoretical stage in the Turkish health system, for a more effective provision of HLC services at the local level, Bursa Osmangazi HLC did further work on the MoH HLC guidelines and developed local algorithms, defined specific roles and responsibilities for the staff involved, and developed its own data collection forms (Table 6). This practice needs to be scaled up and standardized for a balanced implementation throughout the country.

\subsubsection{RECOMMENDATIONS ON INTEGRATION WITHIN PRIMARY CARE LEVEL}

Use local-level good practices to develop nationally standardized pathways and protocols. The efforts of Bursa Osmangazi HLC on guidelines, algorithms, roles, and responsibilities for the HLC staff can be used as an initial attempt to develop guidelines and protocols. As a next step, these guidelines and protocols need to be standardized to ensure a uniform implementation throughout the country. The development and standardization process should be shaped with a bottom-up approach so that the actual implementation issues of the field are reflected to the documents for more effective and easier use. Incorporating the feedback from the field to these documents will also help reduce/eliminate the gap between the central and local needs in terms of the process and results.

Promote and encourage the use of the UHTS-NCD module. The UHTS-NCD module (described in Section 3.4) incorporates a user-friendly design and interface that allows the users (FMPs) to immediately access all 
patient data. ${ }^{211}$ The design also automatically identifies the required pathway to follow based on the interventions done and diagnosis made. These features make the module easy and convenient to use. All these positive features of the UHTS-NCD module should be systematically and properly communicated to the FMPs to encourage them to use the system. As the $\mathrm{MoH}$ expects to increase and stabilize the standard screening process and data entry to the system in the early years of the implementation (around the first 3 years), the procedures can be refined by the experience gained during these initial years.

Authorize non-physician staff access and use of UHTS-NCD modules. The implementation of UHTS-NCD modules will also strengthen evidence-based point-of-care delivery. However, there are two important challenges regarding implementation of evidence-based practice that will impede successful implementation: (a) the limitation of access to the UHTS-NCD modules by multidisciplinary teams (module currently focused on family medicine physicians), and (b) the lack of a system to monitor compliance regarding the application of CPGs by point-of-care staff. The GDHIS might consider improving the information systems to address these limitations in the future, in collaboration with the General Directorate of Public Health.

\subsection{USE OF TECHNOLOGY}

\subsubsection{HEALTH INFORMATION SYSTEMS}

\subsubsection{OVERVIEW AND COUNTRY EXAMPLES}

Technology (HIS in particular) focuses on efficient collection of health system and patient data and on the best use of these data to establish/improve the coordination of patient-provider and intra-provider clinical activities. HIS also concentrate on the use of these data for research, planning, and management decisions about integrated care. Health care providers continuously need patient/beneficiary information in terms of their wants and needs and other providers' care details to adjust or to complement the ongoing patient's care scheme, and achieving efficient integrated care is possible only with efficient communication and information. Therefore, HIS have a critical role in care quality improvement, cost reduction, and establishing of innovative integrated care models. ${ }^{212}$ The experience and research from other industries show that successful integrated supply chain practices are now possible with the advances in technology and these practices do in fact provide inspiration to build new integrated care models that promote more networking and coordination and provide a degree of autonomy for these networked units. Technology also helps transition to integrated care, by pursuing the principle of 'moving the data, not the patient' and by considering ways to reduce unnecessary and unwanted visits to health care providers. ${ }^{213}$

A number of critical factors should be considered for the success of HIS in the integrated care context. First, technology should enable data exchange where all involved actors are able to receive and send data, their systems are interoperable, and unauthorized access to these data is prevented with high security features. Second, for a more person-centered care, technology should engage beneficiaries and caregivers to clearly specify their needs and goals, thus allowing both themselves and their providers to monitor, assess, and review as necessary their care plans. Third, technology should engage its users in the co-design work and

\footnotetext{
${ }^{211}$ Interview with the Head of Chronic Diseases and Elderly Care Department, April 9, 2019.

212 Øvretveit 2017.

213 Øvretveit 2017.
} 
implementation of the integrated care model. This will allow system designers to understand clinical and social care practice and context to incorporate necessary customization to the model..$^{214}$

The use of virtual care methodologies is also important in establishing an effective and efficient NCD management system. Traditionally, access to health care services has been provided as face-to-face services creating an overwhelming burden for the primary care delivery system. Studies show that the primary care physician does not have enough hours in a day to effectively manage all the chronic disease needs of the registered patients to which he/she is responsible and accountable. ${ }^{215} \mathrm{An}$ emerging method for delivering primary care service, that is well suited to the care of NCDs, is virtual care. Virtual care includes modes such as telephone, tele-video, and secure messaging/texting. Virtual care has proven to be an effective method in providing patients with ready access to primary care services and reducing the overwhelming burden of faceto-face care delivered by the physician and has demonstrated excellent outcomes in delivering high-quality care. ${ }^{216} \mathrm{~A}$ foundational concept of managing NCDs through virtual care includes participation by members of the multidisciplinary primary care team with the knowledge, skills, and abilities to share the care of the patient across the team reducing the need for face-to-face care with the physician. Virtual care delivery provided by other disciplines spreads the care for NCDs among other team members and reserves the physician's time to deliver face-to-face care for complex cases and leading medical care for registered patients. The literature describes many different PHC multidisciplinary team models that include doctors, nurses, pharmacists, social workers, dieticians, mental health specialists, diabetic educators, and so on. ${ }^{217}$. However, in each model, members of the health care team have well-defined roles and functions, work to the top of their license and scope, and address system and workflow redesign to optimize care for the population served. ${ }^{218}$

The Zio scheme of the Netherlands is a good example for using technology and information systems for integrated care. One of the factors that facilitated the integrated care efforts under Zio was the commitment to invest in health information technology to track performance. The collection and dissemination of data to care providers is a means to support them in exploring opportunities for continuous improvement of health care provision. To improve care coordination and communication, Zio uses disease-specific electronic patient records including checkup and referral data. These records link laboratory data and clinical measurements and allow information sharing, protocol standardization, and regular collection of user feedback. For diabetes care, Zio has a clinical DSS based on nationally defined standards. ${ }^{219}$

Germany's Gesundes Kinzigtal scheme uses system-wide electronic patient records that can be accessed by partner providers. Data storage is done with high encryption and provider access to patient data (all relevant diagnoses and treatment information) is through a key card offered by the patient. The patient is the owner of his/her own health data and records and has the right to give consent to data disclosure to providers. This data

\footnotetext{
214 Øvretveit 2017.

215 Bodenheimer and Pham 2010; Ghorob and Bodenheimer 2012.

216 Treating diabetes takes more than insulin: Senegal mobile phone project promoting public health. https://www.who.int/en/news-room/feature-stories/detail/treating-diabetes-takes-more-than-insulin-senegal-mobilephone-project-promoting-public-health; WHO 2015, 2016 a.

217 Wootton 2012.

218 SCIE 2018.

${ }^{219}$ Hubertus et al. 2017.
} 
sharing process presents a quick and accurate data communication between care providers as it helps reduce medication interactions, avoid costly and sometimes risky unnecessary services. ${ }^{220}$

Basque Country is another example where integrated care is facilitated by information and communication technologies. Electronic health record (EHR) system for the primary care has been introduced in 1998 and currently covers all PHC level. EHR includes data on patient diagnoses, prescriptions, laboratory test results, referrals, and issued sick-leave certificates. The shared EHR model (Osabide Global) was designed and piloted in 2010 and assumed full implementation since 2013. Osabide Global allows health care providers to access all relevant patient data, have a comprehensive idea about the patient status, and guide the decision making for the treatment and care. Since 2012, patients can arrange remote appointments with health professionals through the multichannel health service center (Osarean). Osarean arranges e-health service provision, health advice, prescription support, and remote appointment setting through web access and SMS. ${ }^{221}$

Basque citizens have access to their own clinical data. Electronic prescription system, business intelligence (BI) tools, and self-assessment instruments (such as Assessment of Readiness for Chronicity in Health Care Organizations, ARCHO) at different levels of the health system are other ICT tools that are being used in the Basque integrated care scheme.222

\subsubsection{CURRENT STATE IN TURKEY}

HIS in Turkey are in an advanced state, and they have significant capabilities to collect and report data. The following paragraphs explain various features of the system with its strengths as well as areas where there is room for further improvement.

Sharing of patient EHRs. Individual-level patient data are collected through different information systems operating under the MoH. These systems are the Family Medicine Information System (Aile Hekimliği Bilgi Sistemi, AHBS) used by FMPs; Public Health Information System²23 (Halk Sağlığı Yönetim Sistemi, HSYS) used by primary care institutions; HBYS used by public hospitals; Dental Health Information System (DHBS); Medical Examination Information System (Muayene Bilgi Yönetim Sistemi, MBYS) under HSYS used by health care institutions including FMCs, FMPs, CHCs, and other FM staff; records data on diagnosis, prescription, medical report, referral and medical interventions done as well as type of examination (emergency, follow-up, normal consultation, and so on); Dialysis Management Information System (Diyaliz Bilgi Yönetim, Sistemi DYOB) used by public, private, and university dialysis centers; and Occupational Medicine Information System (İşyeri Hekimliği Bilgi Sistemi, ISBS).

Individual-level patient data collected from these information systems are kept on the e-pulse platform in the Turkish health system. E-pulse is a cloud-based system collecting data from private and public providers, mobile devices, and the Internet. While all public hospitals are integrated with e-pulse, university hospitals and private hospitals provide data to e-pulse on a voluntary basis. As a default, e-pulse is open to family physicians,

${ }^{220}$ Hildebrandt et al. 2010.

https://www.ncbi.nlm.nih.gov/pmc/articles/PMC2914874/pdf/ijic2010-2010046.pdf.

221 Solinis et al. 2013.

222 https://ec.europa.eu/research/innovation-union/pdf/active-healthy-ageing/llano.pdf.

223 HLC modules operate under the HSYS. As of November 19, 2018, HLC sub-modules that are operational are school health, cancer, preventive dental health, and nutrition and physical activity. HLC sub-modules for which the software is ready and tested and which are ready to be operational are psychological support and social worker services. HLC submodules for which the software is to be developed are physiotherapy services and substance control. 
that is, family physicians have access to individual patient data stored in e-pulse. Other health care providers (including HLC care providers and ER) can access individual patient data with the patient's consent. The patient can limit or extend access privileges to his/her data (for one time, for a period of time, or for always) to one, some, or all providers. ${ }^{224}$ Patients also have the option not to share their data with anyone, but the statistics suggest that this number is small. ${ }^{225} \mathrm{E}$-pulse stores patient historical data including diagnosis, prescriptions, medical surgical history, imaging and reports, lab test results, medications used, and epicrisis ${ }^{226}$ reports (if entered by the physician). Patient-specific data can be seen at the FM level by all family physicians, but family physicians do not have access to hospital data. Similarly, hospitals do not have access to FM data.

There is high interest in consulting patient historical data through e-pulse and the interest of primary care providers is significant. The e-pulse utilization figures in Table 7 suggest that on the one hand there is a trusting relationship between the primary care provider and the patient (patient acknowledges the PHC) and on the other hand the e-pulse is getting acceptance from the primary care providers to access data. This high level of utilization of e-pulse also supports a culture and concern for ensuring patient safety.

\section{Table 7}

\begin{tabular}{|l|l|}
\hline \multicolumn{2}{|l|}{ E-pulse utilization statistics as of November $\mathbf{2 8 ,} \mathbf{2 0 1 8}^{\mathbf{2 2 7}}$} \\
\hline $\begin{array}{l}\text { Current active e-pulse users (those who signed } \\
\text { up) }\end{array}$ & 8.5 million \\
\hline $\begin{array}{l}\text { Share of FMPs who visited e-pulse within total } \\
\text { number of providers who visited e-pulse }\end{array}$ & $\begin{array}{l}68.82 \% \\
(20.059 \mathrm{FMP} \text { visits out of } 29.146 \text { total provider visits) }\end{array}$ \\
\hline $\begin{array}{l}\text { Share of FMP visits within total number of } \\
\text { provider visits }\end{array}$ & $\begin{array}{l}92.28 \% \\
(2,948.153 \mathrm{FMP} \text { visits out of total 3,194.920 all provider visits }\end{array}$ \\
\hline
\end{tabular}

DSS from an integrated care perspective. The main data source for individual-level health data is e-pulse and the data are updated near real time. Data collected through e-pulse are transferred through two Structured Query Language ${ }^{228}$ (SQL) servers: Microsoft SQL Server (MSSQL) (for individual data) and Couchbase (for anonymous individual data). Individual-level historical data are currently kept on three reporting platforms (Oracle BI, Power BI, and Statistical and Causal Analysis in Health [SINA - a national platform developed by the $\mathrm{MoH}]$ ) but the $\mathrm{MoH}$ intends to soon transfer all systems under SINA. Data processing and data analysis are done at the data warehouse level (through Oracle, MSSQL, and Big Data). This configuration/setting does not impose any analysis/data processing burden on e-pulse thus making the system flexible, agile, and efficient.

$\mathrm{MoH}$ information systems impose several features to prevent faulty data entry. Physicians have a time window to enter the patient data to the system, within 30 minutes to 2 hours after medical examination of the patient, or the system does not allow them to enter data for that patient. The system includes a cross-check process on

\footnotetext{
224 The exception is the MCH-related data as sharing this information across the levels of care is a requirement for primary care.

225 The number of citizens who closed their e-pulse data to everyone is around 4,000 people out of 81 million.

${ }^{226}$ A critical or analytical summing up especially of a medical case history (https://www.merriamwebster.com/medical/epicrisis).

227 Data provided by GD HIS of MoH as of November 28, 2018.

$228 \mathrm{SQL}$ is a standard computer language for relational database management and data manipulation. SQL is used to query, insert, update, and modify data. Most relational databases support SQL, which is an added benefit for database administrators (DBAs), as they are often required to support databases across several different platforms. (https://www.techopedia.com/definition/1245/structured-query-language-sql)
} 
the number of patients seen in a hospital and the number reported by physicians in the hospital. This guarantees valid and reliable data in the system. To ensure the authenticity of the imaging data and to authorize reimbursement, there are checks with the Social Security Institution, cross-checks of hash values, and mapping of the person with the image.

The DSS of the MoH has capabilities for risk stratification, population targeting, and disease prioritizing which are important elements of integrated care. The DSS can populate the numbers of any new or prevailing NCD cases (prevalence and incidence data) for a given time interval; on different disaggregation levels (such as FMC, district, province, regional, or national level); by gender and age and by upper and lower diagnosis groups. Another example of DSS capabilities for NCD management is that the DSS can populate data on diagnoses based on International Statistical Classification of Diseases and Related Health Problems 10th Revision (ICD) 10 codes, ACSC admissions and readmissions within 30 days to hospitals (by different disaggregation levels such as by hospital and length of stay) and ED visits (by hospital and by triage color). While the DSS allows constructing different queries and generate reports based on these queries, the system does not currently allow the family physicians or other care providers to develop customized queries and generate reports. Reports are currently developed centrally, based upon formal request. The DSS also uses different BI tools for reporting and analysis. In the future, the $\mathrm{MoH}$ intends to make the health data accessible to the local users through the BI tool. As the head of the HIS department indicates, "current MoH software and the database structure has enough flexibility for analysis, reporting and visual representation." Therefore, it can be concluded that the system has the capability to allow the users to access data and to craft data requests/queries and generate their own reports based on these queries for policy planning.

UHTS and NCD management. In 2019, the MoH intends to launch a new nationwide information system module called UHTS. NCD management will be a sub-module of UHTS and once it is operational, UHTS-NCD will constitute an important element of NCD care and management. UHTS-NCD's goal is to integrate services among FMC, HLC, and secondary care regarding NCD care/management. As the head of the Chronic Diseases and Elderly Care Department indicates, currently no distribution of roles and responsibilities have been made concerning NCD care planning and implementation. The MoH intends to increase patient demand for primary care and integrate care among different providers as well as to shift the bulk of the care from costly secondary care to primary care. The process flow for each chronic disease has been designed end to end (across different levels of care) in UHTS-NCD and the referral chain is intended to be operational under UHTS in the future. The first module of UHTS-NCD is expected to be operational in 2019 and includes HT, DM, obesity, and CVD risk assessment, as these conditions have been prioritized because of their high disease burden. The second module of UHTS-NCD will include the conditions of kidney failure, stroke, and coronary diseases. The third module will include COPD, asthma, and elderly care. The $\mathrm{MoH}$ expects that the second and third modules will be operational by 2023 .

UHTS-NCD is a system to be used by family physicians. To allow family physicians to proactively manage their registered NCD patients and to monitor their own progress and performance, the UHTS-NCD module is able to provide specific clinical data (such as list of patients whose $\mathrm{HbA1c}$ is greater than 9 and whose blood pressure is greater than 140/90). The individual-level patient data can be aggregated on different levels such as FMC/district/provincial/regional and national levels. To complement UHTS-NCD, other patient-specific historical information such as ICD-10 diagnosis code, date, and results of current and previous lab tests trended over time is also available through e-pulse. This will allow the family physicians to proactively manage NCD patients. While UHTS-NCD will have capabilities to allow physicians to have a proactive role in the control and management of NCDs, it is important to note that other PHC or HLC team members' (other than family physicians) lack of access to UHTS-NCD will severely limit integrated care and teamwork. 
Two of the screens designed for UHTS-NCD are noteworthy from an integrated care perspective. One of the screens will provide the patient summary info (for example, on this screen the physician has a patient list and can see how many of the patients have high BP). This would allow the physician to stratify the patients. The other screen will show the physician status (performance) compared to the nationwide performance on selected care interventions. This way the physician will be able to locate himself/herself against the national performance and will elaborate ways to improve his/her performance. On a more granular level, it is advised to also include performance data at the FMC, district, and provincial levels.

To facilitate NCD patient follow-ups, the UHTS-NCD module will have reminders and pop-ups for physicians. Clinical NCD guidelines related to UHTS-NCD are ready and pilot tested. The results of the pilots suggest that family physicians can use them if their registered population is around 2,000 people. The new UHTS-NCD module is to be pilot tested in four provinces ${ }^{229}$ where the registered population is around 2,000 people. Pilots will test the effectiveness of the positive incentives for NCD goals and NCD management; however, the details of the incentive structure have not yet been specified. For a more effective NCD management at the FM level, the MoH also intended to increase the number of FM staff (nurses or other support staff) to two by 2023.

While the current AHBS only maintains the NCD diagnosis data, the new UHTS-NCD module will maintain the data on the medical examination or lab tests concerning the NCDs. Additionally, UHTS-NCD will not only keep track of the patients but also the healthy population, that is, it will take the entire records of population health. It will then guide the physician on the next actions/interventions for each patient. To illustrate, a person with high BP will be monitored four times a year as a mandatory procedure. The patient will then be oriented to HLCs for diet and lifestyle changes. All family physicians will be trained and certified on the new UHTS-NCD module guidelines.

One good prevailing practice of proactive NCD management in the Turkish system is the actual cancer screening and at-risk population detection by family physicians. This actual practice can be thought as an implementation example for UHTS-NCD actual operation. In the current cancer screening and population identification practice, there are two ways through which family physicians learn about the at-risk population: they either look at the specific screens developed by the HIS department ${ }^{230}$ or they use the web service provided within the AHBS. ${ }^{231}$ Interviewees from the HIS department think that the new UHTS-NCD module would operate the same way as the cancer screening and detection practices.

Challenges of using current HIS in the field. While HIS has capabilities and robust structure to address many requirements of integrated care, there are some implementation issues in the field that need to be addressed. Field visit interviews to $\mathrm{HLCs}^{232}$ revealed that there are gaps between the $\mathrm{MoH}$ database structure and data requirements and actual data requirements and reporting and analysis needs of the field. One example is the content of required data: the MoH collects data on first visits to HLCs but HLCs are interested in data of all patient visits (first visits or follow-up visits) as this continuous data is a way for HLC staff to observe the patient status and progress through HLC services in time. Differing interests on continuous data is also apparent in the

\footnotetext{
229 These provinces are identified as Malatya, Edirne, Bartın, and Silivri.

$23030-35$ percent of family physicians are using these screens (as per the meeting with the HIS department).

231 65-70 percent of family physicians are using this web server within the AHBS.

232 Interviews with the representatives of Bursa Osmangazi HLC.
} 
data collection periods of the MoH and the needs of the field. The MoH collects data quarterly whereas HLCs would like to collect data monthly for a more continuous monitoring. ${ }^{233}$

Another gap between the MoH and HLC interests on content is that the MoH mainly collects utilization data. But HLCs need additional data fields in the system that would help them do their own planning and analysis. Interviewees from Bursa Osmangazi HLC suggest that such additional fields can be optional in MoH databases as and those who enter data in those fields can make analysis later.

The visibility/accessibility of existing system data by HLCs is another challenge. Interviewees from Bursa Osmangazi HLC indicate that although data are entered into the system, they are not able to access the data under certain circumstances. One example is the dietitian database which is tied to dietitians, meaning that the individual patient data are kept under the related dietitian. If the dietitian leaves the HLC under which he/she works, the individual patient data tied to him/her also moves with the dietitian and does not stay in the HLC. The HLC therefore loses track of the patient data, at least for a while, until they request for the data from the leaving dietitian (through a formal procedure) or until they request access to the patient data from the HIS department.

Data on the primary care level cannot be viewed and shared among HLCs and FMCs as they use different databases. The HLC database is a module operating under the HSYS and family physicians do not have access to the HSYS. Family physicians use the AHBS and HLCs do not have access to the AHBS, meaning that these two systems do not talk to each other. The HLC database is rather new and does not have enough capabilities for HLC-specific data analysis.

HLCs mainly use two databases to collect patient data: MBYS used by the physicians and HSYS used by the other specialized HLC staff. Currently, these databases do not communicate with each other. There are also gaps in data collection for the multidisciplinary HLC functions: neither of these databases have features allowing psychologists and physiotherapists to collect data. Additionally, no policlinic data entry exists for diabetes patients who are followed up by a dietitian. In other words, HLC staff are not able to see diabetes patients' data whose follow-up is done by a dietitian. Given these challenges, together with the need to share data within the HLC among the staff for the multidisciplinary work, interviewees in Bursa Osmangazi HLC indicate that they developed their own internal model to collect data for all specializations in the HLC and to meet local needs for measurement and performance for strategic planning. ${ }^{234}$ With this internal setting, all HLC units can see all patient data. For this arrangement to work, they take the patient's consent to share data among HLC staff. Bursa Osmangazi HLC also has its own data-keeping practices for clinical indicator follow-up through which it aims to develop the NCD map of the neighborhood.

Interviewees from Bursa Osmangazi HLC indicate that data fields that need to be present in the information system are patient registry at arrival, patient examination information, general policlinic information, and referral information. All units in the HLC should be able to enter data into the system. For continuity of care, this information also needs to be seen by FMCs.

Similar to Bursa Osmangazi HLC, each specialist staff in Hatay HLC keeps his/her own activity data. Hatay HLC interviewees indicate that the $\mathrm{MoH}$ collects data on the number of patients and is interested in the increase

\footnotetext{
${ }^{233}$ A recent feedback from the Department of Public Health Services and Training indicates that HLC data are collected monthly.

234 Osmangazi HLC data collection scheme includes Excel worksheets filled by every specialist HLC staff and HLC physician and HLC common worksheets that combine and report HLC health service data for each patient case.
} 
(that is, volume) in patient numbers. However, the local HLC team needs to collect additional data reflecting the details of their activities to monitor the continuity of care and the progress for each patient.

On the FM side, main difficulties concerning the use of HIS include duplicate/multiple data entry to different modules of the information system for the same content as well as the extensive data that need to be entered to the system by family physicians. The interviewees in Hatay indicated that the data entry takes a significant amount of their time reducing the time they allocate to clinical activity. They emphasize the need for the simplification of the data fields.

3.4.1.3 RECOMMENDATIONS ON INTEGRATION WITHIN PRIMARY CARE LEVEL

The health technology platforms have significantly enhanced Turkey's ability to gather, report, and use provider and population data to foster integration efforts. Optimizing existing technologies and platforms will further promote and support integrated care goals and NCD and prevention care as described in the following paragraphs.

Align the design and intra-connectivity of various databases used at the primary care level. Based on the discussion in the previous section, there are a number of ways for the $\mathrm{MoH}$ to improve the primary care-level databases. First, the $\mathrm{MoH}$ might consider allowing the communication across databases so that primary care providers have access to their patient data in a comprehensive way. Second, the databases should consider and include in the design the needs of the local teams to collect more frequent data. A third issue is that the design and database intra-connection should make sure that patient data stay within the health care unit rather than with the provider. This is especially important for health care staff with high mobility. Last but not the least, the extensive and robust DSS that the MoH currently has should allow for the generation of customized and localized queries and reporting by family physicians as a means to support their patient and population health management plans and strategies. To this end, the $\mathrm{MoH}$ might expand the use of BI tools that it currently uses.

Authorize non-physician staff in FMCs and HLCs access to UHTS-NCD modules and other planned databases. Implementation of UHTS-NCD modules will significantly improve the ability to monitor and proactively manage patients with NCDs. However, as with other software programs either under development or in use, other non-physician staff members in FMCs and/or HLCs do not have full access to efficiently and effectively support NCD management. Limiting access to databases/software presents barriers to team work and a robust, comprehensive, cost-effective NCD management program. It is therefore advised that the technology platform updates consider the idea of giving access to the FMC and HLC staff for database usage.

Consider legitimizing virtual care. It is recommended that the $\mathrm{MoH}$ considers legitimizing virtual care as a means to improve patient access to primary care services for chronic illness needs. This could be accomplished through policy support and implementation guidance development at the $\mathrm{MoH}$ level. The use of virtual care methodologies currently widely available in Turkey is critical in establishing an effective and efficient NCD management system.

Implementation of virtual care for NCDs in Turkey would require, at a minimum, legally defining acceptable modes of care delivery in policy, a designated directorate to take the lead, and/or legislation (as necessary) as well as developing field guidance and training. Providing incentives (financial or otherwise) and developing targets for monitoring and performance of virtual care would be important elements of the implementation plan. Financial and/or workload counting considerations would require careful review and strategizing to optimize widespread implementation efforts.

A practical first step to implementing virtual care is to use the telephone as the method and focus on a specific disease or condition such as diabetes. A project outline would generally include the following steps: establish 
a team of discipline-specific team members, define each team members role, develop specific guidance for patient interaction and care delivery from evidence-based practice guidelines, design the workflow, identify patients with suboptimal clinical indexes $(\mathrm{HbA1c})$ from a group of primary care registered patients, socialize concept with patients and other PC staff/key stakeholders, develop metrics for monitoring performance and evaluation, set project timeline, revise process/plan as needed, share results and lessons learned. The learning experience from the project could be used to inform policy and guidance development for a wider spread.

Continued development and focus on implementation of point-of-care technology. Supporting patient management at the point of care can incentivize the primary care providers and teams (through facilitating and streamlining the work) in their efforts for proactive NCD management. The implementation of the UHTS-NCD modules will begin to revolutionize NCD and care management at the point of care because they are designed to support clinical staff in their implementation efforts. It is strongly recommended that these modules continue to be revised and refined on an ongoing basis. Feedback from end users and subject matter experts should shape future versions and technology capabilities. Important technology components for streamlining and improving care delivery include automated prompts for needed intervention or management, care and prevention management reminders based on individual patient needs, additional features added to the clinical and administrative DSS that emphasize quality and cost-effectiveness. Multidisciplinary team care documentation reflecting delivered health care advice and interventions can be included in the patient electronic medical records or in other active user interfaces. 


\section{SUGGESTED ROADMAP FOR INTEGRATED CARE EFFORTS IN TURKEY}

Table 8 gives the summary of recommendations with their priority levels and timeline for implementation.

\section{Table 8}

\begin{tabular}{|c|c|c|c|}
\hline $\begin{array}{l}\text { Essential } \\
\text { Element }\end{array}$ & Recommendation & Time Frame & Priority \\
\hline \multirow{2}{*}{$\begin{array}{l}\text { Engaged } \\
\text { Leadership }\end{array}$} & Have a champion for integration & $\begin{array}{l}\text { Short term, } 2019 \text { to } \\
\text { early } 2020\end{array}$ & High \\
\hline & $\begin{array}{l}\text { Develop a complementary detailed } \\
\text { integrated care strategy/policy }\end{array}$ & $\begin{array}{l}\text { Medium term, } 2020 \text { or } \\
2021\end{array}$ & High \\
\hline \multirow{3}{*}{$\begin{array}{l}\text { Patient Focus } \\
\text { and Engagement }\end{array}$} & $\begin{array}{l}\text { Develop/disseminate a nationwide } \\
\text { comprehensive health literacy program }\end{array}$ & $\begin{array}{l}\text { Short to medium term, } \\
\text { 2019-2021 }\end{array}$ & Medium to high \\
\hline & $\begin{array}{l}\text { Develop/implement a nationwide program } \\
\text { to improve patient engagement }\end{array}$ & $\begin{array}{l}\text { Short to medium term, } \\
\text { 2019-2021 }\end{array}$ & Medium to high \\
\hline & Establish patient advisory boards & $\begin{array}{l}\text { Short to medium term, } \\
\text { 2019-2021 }\end{array}$ & Medium to high \\
\hline \multirow{2}{*}{$\begin{array}{l}\text { Regulatory } \\
\text { Frameworks }\end{array}$} & $\begin{array}{l}\text { Develop regulations based on the Strategic } \\
\text { Plan objectives }\end{array}$ & Medium term, 2020 & Medium to high \\
\hline & Reward compliant behavior & $\begin{array}{l}\text { Long term, } 2021 \\
\text { onwards }\end{array}$ & Medium \\
\hline \multirow{2}{*}{$\begin{array}{l}\text { Create Enabling } \\
\text { Environment }\end{array}$} & $\begin{array}{l}\text { Increase communication, marketing, and } \\
\text { socialization strategies }\end{array}$ & $\begin{array}{l}\text { Short to medium term, } \\
2019-2021\end{array}$ & Medium \\
\hline & $\begin{array}{l}\text { Establish a committee or consortium } \\
\text { focused on best practices }\end{array}$ & Medium term, 2021 & Medium \\
\hline \multirow{3}{*}{$\begin{array}{l}\text { Alignment of } \\
\text { Finances and } \\
\text { Incentives }\end{array}$} & Develop an ROI evaluation system & $\begin{array}{l}\text { Short term, } 2019 \text { to } \\
\text { early } 2020\end{array}$ & High \\
\hline & Update performance-based contracts & $\begin{array}{l}\text { Short to medium term, } \\
\text { 2019-2021 }\end{array}$ & Medium \\
\hline & $\begin{array}{l}\text { Develop and implement team-based } \\
\text { incentives }\end{array}$ & $\begin{array}{l}\text { Long term, } 2021 \\
\text { onwards }\end{array}$ & Medium to high \\
\hline \multirow{3}{*}{$\begin{array}{l}\text { Monitoring and } \\
\text { Performance } \\
\text { Improvement }\end{array}$} & $\begin{array}{l}\text { Use key proxy indicators and process } \\
\text { indicators to measure the effectiveness of } \\
\text { PHC on NCDs }\end{array}$ & Short term, 2019 & High \\
\hline & $\begin{array}{l}\text { Incorporate NCD prevention and control } \\
\text { into the workload of family physicians by } \\
\text { reducing their registered population }\end{array}$ & $\begin{array}{l}\text { Short to medium term, } \\
2020\end{array}$ & High \\
\hline & $\begin{array}{l}\text { Provide transparent reporting of critical } \\
\text { health care metrics }\end{array}$ & $\begin{array}{l}\text { Short to medium term, } \\
2019 \text { or } 2020 .\end{array}$ & High \\
\hline
\end{tabular}




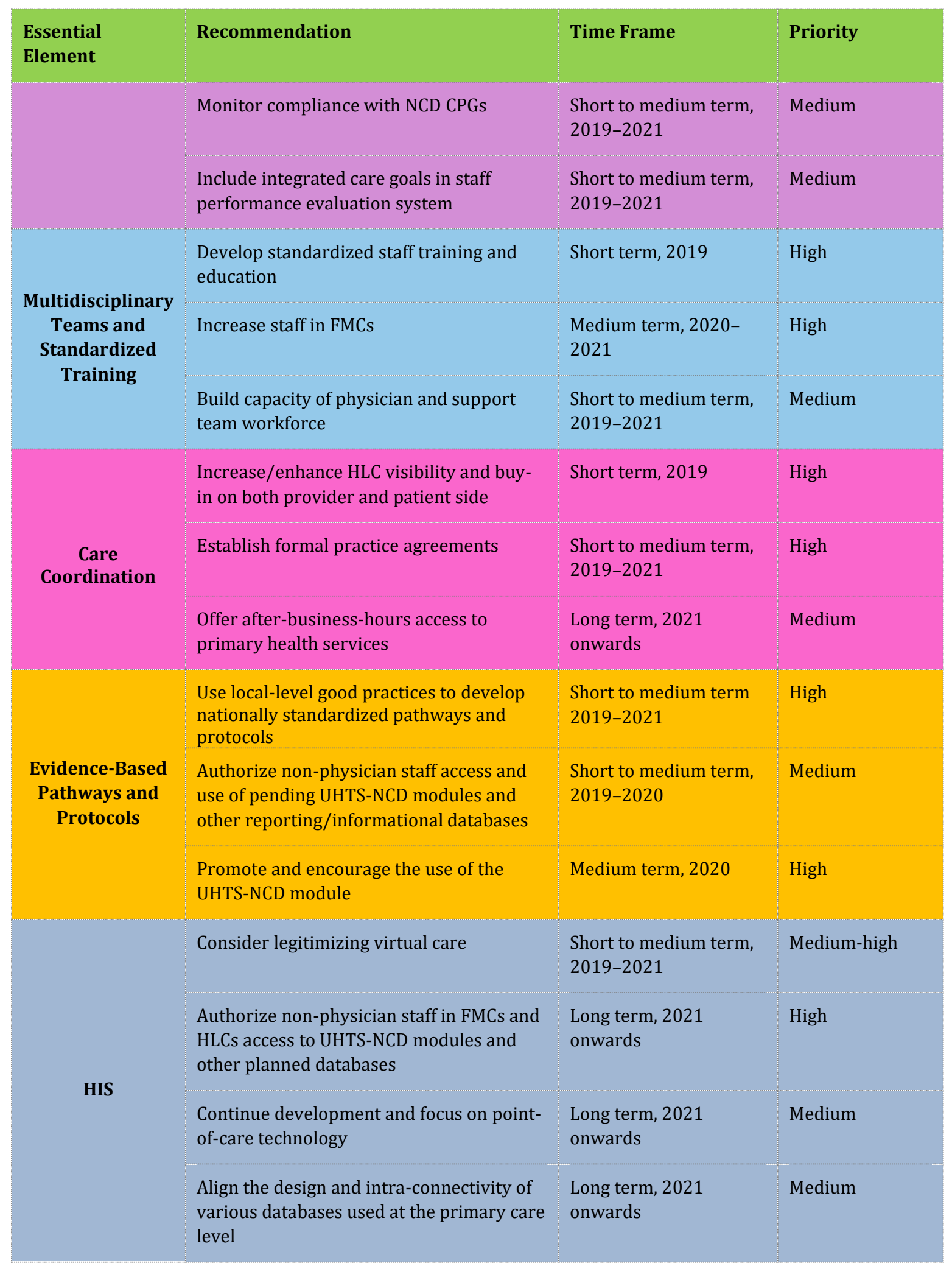




\section{CONCLUSION}

A closer look at the Turkish health system from the central policy making and the local implementation perspectives reveals that the system has both strong aspects and gaps to be addressed for its journey toward integrated care.

Low health literacy among population, absence of links between registered patient registries among different levels of care, absence of monetary and non-monetary incentives, absence of concrete coordination mechanisms and multidisciplinary work protocols are the main challenges to be addressed by the MoH. The presence of a robust and flexible IT system and the initial efforts in the field on areas including care coordination, multidisciplinary work, leadership engagement are remarkable enablers to take the integrated care efforts forward.

As has been detailed in the recommendations section, possible future actions concentrate on the establishment of executive level advisory structures and leadership for integrated care to ensure standardized integrated care policy development and implementation, building of effective communication and socialization mechanisms to get the buy-in and support of stakeholders, improvement of health workers' skills and capacity as well as increasing the nursing staff numbers in the primary care settings, introduction of non-fiscal care coordination agreements among the primary care provider entities, development and implementation of performance criteria at each level of care concerning integrated care, expanding the access of the upcoming UHTS-NCD module to non-physician staff, and consideration of virtual care as a new policy toward integrated care.

The road map suggested under Section 4 lists some major actions together with their priority level and possible timeline for implementation. The road map in that sense might be a preliminary broad guide to the $\mathrm{MoH}$ in their efforts to plan for integrated care. 


\section{BIBLIOGRAPHY}

Advisory Board. 2014. "Build the All Access Medical Group: Meeting Consumer Demands to Ensure

Sustainable Growth.” http://www.advisory.com/research/medical-group-strategy-

council/events/webconferences/2014/building-the-all-access-medical-group.

Agency for Healthcare Research and Quality. 2013. "Creating Capacity for Improvement in Primary Care: The Case for Developing a Quality Improvement Infrastructure."

https://www.ahrq.gov/professionals/prevention-chronic-care/improve/capacity-building/pcmhqi1.html.

Augustine, Matthew R., Karin Nelson, Stephan D. Fihn, and Edwin S. Wong. 2016. "Is Patient-Reported Access to VHA Services Associated with Preventable Hospitalizations?" J Gen Intern Med.

Barker, P. M., A. Reid, and M. W. Schall 2016. “A Framework for Scaling Up Health Interventions: Lessons from Large-Scale Improvement Initiatives in Africa." Implementation Science 11: 12. doi:10.1186/s13012-0160374-x. http://www.ncbi.nlm.nih.gov/pmc/articles/PMC4731989/.

2018. "The Effects of Integrated Care: A Systematic Review of UK and International Evidence." BMC Health Services Research 18: 350 https://doi.org/10.1186/s12913-018-3161-3.

Berchet, C., and C. Nader. 2016. "The Organisation of Out-of-Hours Primary Care in OECD countries." OECD Health Working Papers 89, OECD Publishing, Paris. http://dx.doi.org/10.1787/5jlr3czbqw23-en.

Bertram, M. Y., K. Sweeny, J. A. Lauer, D. Chisholm, P. Sheehan, B. Rasmussen, S. R. Upreti, L. P. Dixit, K. George, and S. Deane 2018. "Investing in Non-Communicable Diseases: An Estimation of the Return on Investment for Prevention and Treatment Services." Lancet Taskforce on NCDs and Economics 391 (10134): 2071-2078. https://doi.org/10.1016/S0140-6736(18)30665-2.

Best, A., T. Greenhalgh, S. Lewis, J. E. Saul, S. Carroll, and J. Bitz. 2012. "Large-System Transformation in Health Care: A Realist Review." Milbank Quarterly 90 (2012): 421-4.

https://www.ncbi.nlm.nih.gov/pmc/articles/PMC3479379/pdf/milq0090-0421.pdf.

Bidassie, B. 2014. "VA Experience in Implementing Patient-Centered Medical Home Using a Breakthrough Series Collaborative." J Gen Intern Med 29 (Suppl 2):S563-71. doi:10.1007/s11606-014-2773-5.

Blumenthal, D. M., Z. Song, A. B. Jena, and T. Ferris. 2013. “Guidance for Structuring Team-Based Incentives in Health Care." American Journal of Managed Care 19 (2): e64-e70.

https://www.ncbi.nlm.nih.gov/pmc/articles/PMC3984877/.

Bodenheimer, T., A. Ghorob, R. Willard-Grace, and K. Grumbach. 2014. "The 10 Building Blocks of HighPerforming Primary Care." Annals of Family Medicine 12: 166-171. doi:10.1370/afm.1616.

Bodenheimer, T., and H. H. Pham. 2010. "Primary Care: Current Problems and Proposed Solutions." Health Affairs 29: 799-805. https://www.healthaffairs.org/doi/10.1377/hlthaff.2010.0026.

Borgermans, L., and D. Devroey. 2017. "A Policy Guide on Integrated Care (PGIC): Lessons Learned from EU Project INTEGRATE and Beyond." International Journal of Integrated Care 17 (4): 8.

http://doi.org/10.5334/ijic.3295.

Brook, Robert H. 2015. "Redefining Health Care Systems.” RAND.

https://www.rand.org/pubs/corporate pubs/CP788.html.

Bruce, D., and B. Perry. 2015. “Community-Oriented Integrated Care: A Scottish Perspective." London Journal of Primary Care7: 44-48. https://www.ncbi.nlm.nih.gov/pmc/articles/PMC4494465/pdf/LJPC-07-044.pdf. 
Burgess, Lizzy. 2016. "SPICe Briefing, Integration of Health and Social Care." The Scottish Parliament. http://www.parliament.scot/ResearchBriefingsAndFactsheets/S5/SB 16-

70 Integration of Health and Social Care.pdf.

Busetto, L., K. Luijkx, A. Huizing, and B. Vrijhoef. 2015. "Implementation of Integrated Care for Diabetes Mellitus Type 2 by Two Dutch Care Groups: A Case Study." https://doi.org/10.1186/s12875-015-0320-z.

Busetto, Loraine, Katrien Luijkx, Stefano Calciolari, Laura Guadalupe, González Ortiz, Hubertus Johannes, and Maria Vrijhoef. 2018. "Barriers and Facilitators to Workforce Changes in Integrated Care." International Journal of Integrated Care 18 (2): 17. https://www.ijic.org/articles/10.5334/ijic.3587/

Campbell, J., J. Buchan, G. Comett,, B. David, G. Dussault, H. Fogstad, I. Fronteira, L. Rafael, F. Nyonator, A. Pablos-Méndez, E. E. Quain, A. Starrs, and V. Tangcharoensathien. 2013. "Human Resources for Health and Universal Health Coverage: Fostering Equity and Effective Coverage." Bull World Health Organ 91 (11): 853863. doi:10.2471/BLT.13.118729. https://www.ncbi.nlm.nih.gov/pmc/articles/PMC3853950/.

Carrier, E., M. K. Dowling, and H. H. Pham. 2012. "Care Coordination Agreements: Barriers, Facilitators, and Lessons Learned." Am J Manag Care 18 (11): e398-404. https://www.ncbi.nlm.nih.gov/pubmed/23198751. Carter, K., E. Chalouhi, S. McKenna, and B. Richardson. 2011. "What it Takes to Make Integrated Care Work." Health Int 11: 48-55.

https://www.mckinsey.com/ /media/mckinsey/dotcom/client service/healthcare $\% 20$ systems $\% 20$ and $\% 2$ 0services/health\%20international/issue\%2011\%20new\%20pdfs/hi11 48\%20integratedcare noprint.ashx.

Chaiyachati, K. H., K. Gordon, T. Long, W. Levin, A. Khan, E. Meyer, A. Justice, and R Brienza. 2014. "Continuity in a VA Patient-Centered Medical Home Reduces Emergency Department Visits.” PLoS ONE 9 (5): e96356. doi:10.1371/journal.pone.0096356.

Cotlear, D., S. Nagpal, O. Smith, A. Tandon, and R. Cortez. 2015. "GOING UNIVERSAL How 24 Developing Countries Are Implementing Universal Health Coverage Reforms from the Bottom Up.” World Bank Group.

http://www-

wds.worldbank.org/external/default/WDSContentServer/WDSP/IB/2015/09/10/090224b0830cc779/1 0/ Rendered/PDF/Going0universa0s0from0the0bottom0up.pdf.

Curry, N; Harris, M; Gunn, L; Pappas, Y; Blunt, I; Soljak, M; Mastellos, N; Holder, H; Smith, J; Majeed, A; Ignatowicz, A; Greaves, F; Belsi, A; Costin-Davis, N; Jones Nielsen, J; Greenfield, G; Cecil, E; Patterson, S; Car, J; Bardsley, M, 2013. "Integrated Care Pilot in North-West London: A Mixed Methods Evaluation." International Journal of Integrated Care 13 (3) https://dspace.library.uu.nl/handle/1874/278681.

Davis, K., Stremikis, K., Squires, D. and Schoen, C. 2014. "Mirror, Mirror on the Wall. How the Performance of the US Healthcare System Compares Internationally." The Commonwealth Fund.

https://www.commonwealthfund.org/sites/default/files/documents/ media files publications fund repor t 2014 jun 1755 davis mirror mirror 2014.pdf.

Department of Health Australia. 2015. Primary Health Care Advisory Group Final Report: Better Outcomes for People with Chronic and Complex Health Conditions.

https://www.health.gov.au/internet/main/publishing.nsf/Content/76B2BDC12AE54540CA257F72001102B 9/\$File/Primary-Health-Care-Advisory-Group Final-Report.pdf.

Desmedt, M., V. Sonja, J. Hellings, J. Bergs, E. Dessers, P. Vankrunkelsven, H. Vrijhoef, L. Annemans, N. Verhaeghe, M. Petrovic, and D. Vandijck. 2016. “Economic Impact of Integrated Care Models for Patients with 
Chronic Diseases :A Systematic Review. Value in Health 19 (6): 892-906.

https://doi.org/10.1016/j.jval.2016.05.001.

Driscoll, D. L. 2017. "Transforming Primary Care Practice." Content last reviewed March 2017. Agency for Healthcare Research and Quality, Rockville, MD. https://www.ahrq.gov/ncepcr/grants/transforminggrants/profiles/driscoll.html

Dünya Sağlık Örgütü. 2014. Bulaşıcı Olmayan Hastalıklarda Daha İyi Sonuclar: Sağlık Sistemi icin Zorluklar ve Fırsatlar, Türkiye Ülke Değerlendirmesi.

https://sbu.saglik.gov.tr/Ekutuphane/kitaplar/BOH \%C3\%9Clke raporu TR.pdf.

European Summit on Innovation for Active and Healthy Ageing. 2015. "B3 Action Group Maturity Matrix for Integrated Care Presentation." https://ec.europa.eu/research/innovation-union/pdf/active-healthy-

ageing/llano.pdf.

European Union. 2017. "Tools and Methodologies to Assess Integrated Care in Europe."

https://ec.europa.eu/health/sites/health/files/systems performance assessment/docs/2017 blocks en 0.p df. (accessed October 12, 2018).

———. 2018. "A New Drive for Primary Care in Europe: Rethinking Assessment Tools and Methodologies." https://ec.europa.eu/health/sites/health/files/systems performance assessment/docs/2018 primarycare e g en.pdf.

Evans, J. M., A. Grudniewicz, G. R. Baker, and W. P. Wodchis. 2016. “Organizational Context and Capabilities for Integrating Care: A Framework for Improvement." International Journal of Integrated Care 16 (3): 1-14, http://dx.doi.org/10.5334/ijic.2416.

Gesundes Kinzigtal. 2013. “Integrated Care Value Case.” Local Government Association, https://www.local.gov.uk/sites/default/files/documents/kinzigtal-funding-flows-a-3fc.pdf.

- - - 2016. "The Business of Population Health Management." Black Forest, Germany, Global Forum for Health Care Innovators. https://www.gesundes-kinzigtal.de/wp-content/uploads/2017/01/GlobalForum Gesundes-Kinzigtal Case study 2016.pdf.

Ghorob, A., and T. Bodenheimer. 2012. "Sharing the Care to Improve Access to Primary Care." New England Journal of Medicine doi:10.1056/NEJMp1202775.

Gilburt, Helen. 2016. "Supporting Integration through New Roles and Working Across Boundaries. The King's Fund."

https://www.kingsfund.org.uk/sites/files/kf/field/field publication file/Supporting integration web.pdf.

Greenhalgh, T., J. Howick, and N. Maskrey. 2014. "Evidence Based Medicine: A Movement in Crisis?” BMJ 348: 3725. https://doi.org/10.1136/bmj.g3725.

Gulliford, Martin. 2017. “Comment: Access to Primary Care and Public Health." Lancet 2: e532.

https://www.thelancet.com/pdfs/journals/lanpub/PIIS2468-2667(17)30218-9.pdf.

Hacettepe Üniversitesi Nüfus Etütleri Enstitüsü. 2008. Türkiye'nin Demografik Dönüşümü. http://www.hips.hacettepe.edu.tr/TurkiyeninDemografikDonusumu 220410.pdf.

Hamer, S., and G. Collinson. 2005. Achieving Evidence Based Practice E-Book: A Handbook for Practitioners. https://books.google.com/books?id=4d71BQAAQBAJ\&lpg=PP1\&ots=KCpznNNPFE\&dq=evidenced\%20based \%20practice\%20in\%20 primary \%20 care\&lr\&pg=PP1\#v=onepage\&q=evidenced $\% 20$ based $\% 20$ practice $\% 20 \mathrm{i}$ $\underline{\mathrm{n} \% 20 \text { primary } \% 20 \text { care } \& \mathrm{f}=\text { false. }}$ 
Hansen, P. M., D. H. Peters, H. Niayesh, L. P. Singh, V. Dwivedi, and G. Burnham. 2008. "Measuring and Managing Progress in the Establishment of Basic Health Services: The Afghanistan Health Sector Balanced Scorecard." doi:10.1002/hpm.931.

https://www.measureevaluation.org/resources/networks/datausenet/dashboards-and-data-use-forummay-2010/Afghanistan\%20Balanced\%20Scorecard\%20-020IJHPM.pdf.

Hatano, Y., M. Matsumoto, M. Okita, K. Inoue, K. Takeuchi, T. Tsutsui, S. Nishimura, and T. Hayashi. 2017. "The Vanguard of Community-based Integrated Care in Japan: The Effect of a Rural Town on National Policy." International Journal of Integrated Care 17 (2): 1-9. http://dx.doi.org/10.5334/ijic.2451.

Health Care Denmark. 2018. “Denmark - A Telehealth Nation.” White Paper. https://www.healthcaredenmark.dk/media/1625194/HCD-Telehealth-white-paper-v1-single-0318.pdf.

Health Foundation. 2011. "Getting Out of Hospital? The Evidence for Shifting Acute Inpatient and Day Case Services from Hospitals into the Community." https://www.health.org.uk/publications/getting-out-ofhospital.

Hempel, S., Stockdale, S., Danz, M., Rose, D.E., Kirsh, S., Curtis, I., and Rubenstein, L. 2018. “Access Management in Primary Care." RAND.

https://www.rand.org/content/dam/rand/pubs/research reports/RR2500/RR2536/RAND RR2536.pdf.

Heslop, L., R. Power, and K. Cranwell. 2014. "Building Workforce Capacity for Complex Care Coordination: A Function Analysis of Workflow Activity" Human Resources for Health. http://www.human-resourceshealth.com/content/12/1/52.

Hildebrandt, H., C. Hermann, R. Knittel, M. Richter-Reichhelm, A. Siegel, and W. Witzenrath. 2010. "Gesundes Kinzigtal Integrated Care: Improving Population Health By a Shared Health Gain Approach and a Shared Savings Contract." https://www.ncbi.nlm.nih.gov/pmc/articles/PMC2914874/pdf/ijic20102010046.pdf.

Huber, C., O. Reich, M. Früh, and T. Rosemann. 2016. "Effects of Integrated Care on Disease-Related Hospitalisation and Healthcare Costs in Patients with Diabetes, Cardiovascular Diseases and Respiratory Illnesses: A Propensity-Matched Cohort Study in Switzerland." International Journal of Integrated Care https://www.ncbi.nlm.nih.gov/pmc/articles/PMC5015553/pdf/ijic-16-1-2455.pdf

Hubertus, J., A. Huizing, K. Udayakumar, J. Gonzalez-Smith, K. Kadakia, and A. Thoumi. 2017. “Case Study: Zio Integrated Care Network." The Netherlands.

https://healthpolicy.duke.edu/sites/default/files/atoms/files/netherlands 25jan2017.pdf.

Institute for Healthcare Improvement. 2003. “The Breakthrough Series: IHI's Collaborative Model for Achieving Breakthrough Improvement." IHI Innovation Series White Paper. Institute for Healthcare Improvement, Boston.

http://www.ihi.org/resources/ layouts/download.aspx?SourceURL=\%2fresources\%2fKnowledge+Center $+\mathrm{A}$ ssets\%2f364ac260-175b-498d-ae21-8670db1fbc92\%2fIHIBreakthroughSerieswhitepaper2003.pdf.

——_. 2019. "Unique Challenges, Creative Solutions_ Improving Care and Spreading Change in Developing Nations."

http://www.ihi.org/resources/Pages/ImprovementStories/UniqueChallengesCreativeSolutions.aspx. (accessed February 5, 2019).

Letamendi, Iker. "ITHACA (Innovation in Health and Care for All) Project." Interreg Europe https://www.interregeurope.eu/fileadmin/user upload/tx tevprojects/library/file 1536224467.pdf. 
Lewis, Richard Q., R. Rosen, N. Goodwin, and J. Dixon. 2010. "Where Next for Integrated Care Organisations in the English NHS?." The Nuffield Trust. https://www.nuffieldtrust.org.uk/files/201701/where-next-integrated-care-english-nhs-web-final.pdf.

Lyngsø, Anne Marie, Nina Skavlan Godtfredsen, Dorte Høst, and Anne Frølich. 2014. “Instruments to Assess Integrated Care. A Systematic Review." Int J Integr Care 14 https://dspace.library.uu.nl/bitstream/handle/1874/299097/1184-7142-1PB.pdf? sequence=2\&isAllowed $=\mathrm{y}$.

Maruthappu, Mahiben, Ali Hasan, and Thomas Zeltner. 2015. "Enablers and Barriers in Implementing Integrated Care." Health Systems \& Reform 1 (4): 250-256. doi:10.1080/23288604.2015.1077301. https://doi.org/10.1080/23288604.2015.1077301.

Miyata, H., S. Ezoe, M. Hori, M. Inoue, K. Oguro, T. Okamoto, K. Onishi, K. Onozaki, T. Sakakibara, K. Takeuchi, Y. Tokuda, Y. Yamamoto, M. Yamazaki, and K. Shibuya. 2015. “Japan Vision: Health Care 2035.” https://www.ncbi.nlm.nih.gov/pubmed/26122147.

MoH. 2015. "Cost-Effectiveness in Non-Communicable Diseases Note."

-__. 2018. "Guidelines for Healthy Living Centers."

Nelson, K., P. W. Sylling, L. Taylor, D. Rose, A. Mori, and S. D. Fihn. 2017. "Clinical Quality and the PatientCentered Medical Home." Journal American Medical Association

https://www.ncbi.nlm.nih.gov/pubmed/28459952.

Nolan, K., M. W. Schall, F. Erb, and T. Nolan. 2005. "Using a Framework for Spread: The Case of Patient Access in the Veterans Health Administration." http://qi.elft.nhs.uk/wp-

content/uploads/2015/05/15 case study three 08 schall va.pdf.

Nolte, Ellen, and Martin McKee. 2008. "Caring for People with Chronic Conditions: A Health System Perspective." European Observatory on Health Systems and Policies.

http://www.euro.who.int/ data/assets/pdf file/0006/96468/E91878.pdf.

OECD (Organisation for Economic Cooperation and Development). 2016. "Health Workforce Policies in OECD Countries: Right Jobs, Right Skills, Right Places.” OECD, Paris. http://dx.doi.org/10.1787/9789264239517-en.

https://bmcpublichealth.biomedcentral.com/articles/10.1186/1471-2458-14-575.

O’Malley, A. 2012. "After Hours Access to Primary Care Practices Linked With Lower Emergency Department Use and Less Unmet Medical Need." Health Affairs

https://www.healthaffairs.org/doi/pdf/10.1377/hlthaff.2012.0494.

- — 2013. "After-Hours Access to Primary Care Practices Linked With Lower Emergency Department Use And Less Unmet Medical Need." Health Affairs 32 (1): 175-183.

(http://content.healthaffairs.org/content/32/1/175.full.html.

Onder, G., K. Palmer, R. Navickas, E. Jureviciene, F. Mammarella, M. Strandzheva et al. 2015. "Time to Face the Challenge of Multimorbidity: A European Perspective from the Joint Action on Chronic Diseases and Promoting Healthy Ageing Across the Life Cycle (JA-CHRODIS)." European Journal of Internal Medicine 26 (3): 157-9. https://doi.org/10.1016/j.ejim.2015.02.020 Epub 2015/03/24.

Oni, T., N. McGrath, R. BeLue, P. Roderick, S. Colagiuri, and C. R. May et al. 2014. "Chronic Diseases and MultiMorbidity-A Conceptual Modification to the WHO ICCC Model for Countries in Health Transition." BMC 
Public Health 14: 575. Hempel, S. 2018. "Access Management in Primary Care: Perspectives from an Expert Panel." Rand. https://www.rand.org/pubs/research reports/RR2536.html.

Orueta, J., M. Mateos Del Pinoa, I. Berazaa, R. Solinis, M. Zubizarreta, and C. Sarabia. 2013. "Stratification of the Population in the Basque Country: Results in the First Year of Implementation." doi: 10.1016/j.aprim.2012.01.001.

https://www.sciencedirect.com/science/article/pii/S0212656712000182?via\%3Dihub.

Øvretveit, J. 2017. “Digital Technologies Supporting Person-Centered Integrated Care - A Perspective.” International Journal of Integrated Care 17(4): 6. http://doi.org/10.5334/ijic.3051.

Quackenbush, James, Roger Shenkel, and Victoria Schatz. 2004. "Creating a Successful After-Hours Clinic. American Academy of Family Practice." https://www.aafp.org/fpm/2004/0100/p39.html

Raleigh, V., M. Bardsley, P. Smith, G. Wistow, R. Wittenberg, B. Erens, and N. Mays. 2014. Integrated Care and Support Pioneers: Indicators for Measuring the Quality of Integrated Care. Final Report. PIRU Publication 20148. https://piru.lshtm.ac.uk/assets/files/IC and support Pioneers-Indicators.pdf.

Reich, Michael R., and Kenji Shibuya. 2015. "The Future of Japan's Health System — Sustaining Good Health with Equity at Low Cost." N Engl J Med 373 (19): 1793-1797. doi:10.1056/ nejmp1410676

Reinertsen, J. L., M. Bisognano, and M. D. Pugh. 2008. "Institute for Healthcare Improvement. Innovation Series: Seven Leadership Leverage Points for Organization-Level Improvement in Health Care (Second Edition)." IHI Innovation Series White Paper. Institute for Healthcare Improvement, Cambridge, MA. http://www.ihi.org/resources/Pages/IHIWhitePapers/SevenLeadershipLeveragePointsWhitePaper.aspx.

Reinertsen, J. L., A. G. Gosfield, W. Rupp, and J. W. Whittington. 2007. "Engaging Physicians in a Shared Quality Agenda." IHI Innovation Series White Paper, Institute for Healthcare Improvement, Cambridge, MA.

http://www.ihi.org/resources/ layouts/download.aspx?SourceURL=\%2fresources\%2fKnowledge + Center + A ssets\%2fIHIWhitePapers+-+EngagingPhysiciansinaSharedQualityAgenda 146fd2f3-3d61-42ea-af95fdc76761e665\%2fIHIEngagingPhysiciansWhitePaper2007.pdfv

Resmi Gazete, Sayı 29007. 2014. Sağlık meslek mensupları ile sağlık hizmetlerinde çalışan diğer meslek mensuplarının iş ve görev tanımlarına dair yönetmelik https://dosyaism.saglik.gov.tr/Eklenti/12541,20140522-29007-sag-meslek-mensuplari-ile-sag-hizde-caldiger-meslek-mensuplarinin-is-ve-gorev-tanimlarina-dair-yonetmelikpdf.pdf?

Rosland, A. M., E. Wong, M. Maciejewski, D. Zulman, R. Piegari, S. Fihn, and K. Nelson. 2017. "Patient-Centered Medical Home Implementation and Improved Chronic Disease Quality: A Longitudinal Observational Study." Health Services Research 53 (4): 2503-2522. doi: 10.1111/1475-6773.12805.

Safety Net Medical Home Initiative. 2013a. "Engaged Leadership." http://www.safetynetmedicalhome.org/sites/default/files/Implementation-Guide-Engaged-Leadership.pdf. —_- 2013b. "Implementation Guide to Patient Centered Interactions." http://www.safetynetmedicalhome.org/sites/default/files/Implementation-Guide-Patient-CenteredInteractions.pdf.

- - 2014. "Patient-Centred Medical Home Assessment (PCMH-A)." Adapted for use in Australia from the Safety Net Medical Home Initiative. http://www.safetynetmedicalhome.org/sites/default/files/pcmh-a.pdf. Sağlık-Sen. 2014. Türkiye Sağlık Okuryazarlı̆̆ı Araştırması. http://www.sagliksen.org.tr/cdn/uploads/gallery/pdf/8dcec50aa18c21cdaf86a2b33001a409.pdf. 
Sağlık Bilgi sistemleri Genel Müdürlüğ̈ T.C. Sağlık Bakanlığı, Sağlık Hizmetleri Genel Müdürlügü, Sağlıkta Kalite ve Akreditasyon Daire Başkanlığı. 2015. Gösterge Yönetimi Rehberi.

https://dosyamerkez.saglik.gov.tr/Eklenti/3502,gostergeyonetimirehberi-1408151pdf.pdf.

Schottenfeld, L., D. Petersen, D. Peikes, R. Ricciardi, H. Burak, R. McNellis, and J. Genevro. 2016. Creating Patient-Centered Team-Based Primary Care. Rockville, MD: Agency for Healthcare Research and Quality. https://pcmh.ahrq.gov/page/creating-patient-centered-team-based-primary-care.

Schultz, E., N. Pineda, J. Lonhart, S. Davies, and K. McDonald. 2013. "A Systematic Review of the Care Coordination Measurement Landscape.” BMC Health Services Research 13: 119.

http://www.biomedcentral.com/1472-6963/13/119.

SCIE. 2018. "Delivering Integrated Care. The Role of the Multidisciplinary Team."

https://www.scie.org.uk/integrated-health-social-care/measuring-progress/role-multidisciplinary-team.

Selfie 2020. 2016. Work Package 2 Report, Germany. https://www.selfie2020.eu/wp-

content/uploads/2016/12/SELFIE WP2 Germany Final-thick-descriptions.pdf.

Sharfstein, J. M. 2014. “The Strange Journey of Population Health.” Milbank Quarterly 92 (4): 640-3.

https://doi.org/10.1111/1468-0009.12082.

Shi, L., B. Starfield, R. Politizer, and J. Regan. 2002. "Primary Care, Self-rated Health, and Reductions in Social Disparities in Health." Health Services Research 37 (3): 529-550.

https://www.ncbi.nlm.nih.gov/pmc/articles/PMC1434650/.

Social Care Institute for Excellence. 2017. "Developing an Integration Scorecard: A Model for Understanding and Measuring Progress Towards Health and Social Care Integration."

https://www.scie.org.uk/files/integrated-health-social-care/measuring-

progress/scorecard/developing/developing-an-integration-scorecard.pdf.

Solinis, Pérez P., N. Polanco, and C. Quevedo. 2013. “Integrated Care: The Basque Perspective.” International Journal of Healthcare Management 6 (4): 211-216. https://akademia.nfz.gov.pl/wp-

content/uploads/2016/06/IC-Basque.pdf.

Sørensen, K., J. Pelikan, F. Rothlin, K. Ganahl, Z. Slonska, G. Doyle, J. Fullam, B. Kondilis, D. Agrafiotis, E. Uiters, M. Falcon, M. Mensing, K. Tchamov, S. van den Broucke, and H. Brand. 2015. "Health Literacy in Europe: Comparative Results of the European Health Literacy Survey (HLS-EU)."

doi:10.1093/eurpub/ckv043 https://www.ncbi.nlm.nih.gov/pmc/articles/PMC4668324/pdf/ckv043.pdf.

Stein, V. 2016. Integrated Care around the World: Examples to Help Improve (Primary) Health Care in Poland. https://akademia.nfz.gov.pl/wp-content/uploads/2016/12/Raport Opieka koordynowana ENG.pdf.

Stout, S., C. Klucznik, A. Chevalier, R. Wheeler, J. Azzara, L. Gray, D. Scannell, L. Sweeney, M. Saginario, I. Lopes, in collaboration with K. Meisinger, M. Littlefield, and A. Jorgensen. "Cambridge Health Alliance Model of Team-Based Care Implementation Guide and Toolkit."

https://www.samhsa.gov/sites/default/files/programs campaigns/samhsa hrsa/team-based-careimplementation.pdf.

Strandberg-Larsen, M., and A. Krasnik. 2009. "Measurement of Integrated Healthcare Delivery: A Systematic Review of Methods and Future Research Directions." International Journal of Integrated Care 9 https://www.ncbi.nlm.nih.gov/pmc/articles/PMC2663702/pdf/ijic2009-200901.pdf.

Struckman, V. et al. 2017. "Relevant Models and Elements of Integrated Care for multi-morbidity: Results of a Scoping Review.” http://dx.doi.org/10.1016/j.healthpol.2017.08.008. 
T.C. Sağlık Bakanlığı. 2016. Türkiye Sağlık Okuryazarlığı Ölçekleri Güvenilirlik ve Geçerlilik Çalışması, https://sbu.saglik.gov.tr/Ekutuphane/kitaplar/Sa\%C4\%9Fl\%C4\%B1k\%200kur\%20Yazarl\%C4\%B1\%C4\%9 F\%C4\%B1.pdf.

The Economic Intelligence Unit Limited. 2016. “An Integrated Approach to Value-Based Healthcare: Germany's Gesundes Kinzigtal.” http://vbhcglobalassessment.eiu.com/an-integrated-approach-to-valuebased-healthcare-germanys-gesundes-kinzigtal/

The King's Fund. 2017. "Developing Accountable Care Systems: Lessons from Canterbury, New Zealand.” https://www.kingsfund.org.uk/publications/developing-accountable-care-systems.

Tranberg, M., P. Vedsted, B . Hammer, M,. Christensen. S. Birkeland, and G. Moth. 2018. "Factors Associated with Low Patient Satisfaction in Out-of-Hours Primary Care in Denmark-A Population-Based Cross-Sectional Study." BMC Family Practice https://bmcfampract.biomedcentral.com/track/pdf/10.1186/s12875-017-0681-6.

Türkiye Hastalık Yükü Çalışması. 2004. Refik Saydam Hıfzıssıhha Merkezi Başkanlığı. https://sbu.saglik.gov.tr/Ekutuphane/Yayin/166.

Weinick, R. M., R. M. Burns, and A. Mehrotra. 2010. "Many Emergency Department Visits could be Managed at Urgent Care Centers and Retail Clinics”. Health Aff (Millwood) 29 (9): 1630-6. doi:10.1377/hlthaff.2009.0748.

Valentijn, P. P., S. M. Schepman, W. Opheij, and M. A. Bruijnzeels. 2013. "Understanding Integrated Care: A Comprehensive Conceptual Framework Based on the Integrative Functions of Primary Care." Int J Integr Care 13: e010.

WHO (World Health Organization). 2002. Innovative Care for Chronic Conditions, Building Blocks for Action. WHO Global Report. http://www.who.int/chp/knowledge/publications/icccglobalreport.pdf?ua=1.

- - - 2010a. "Health Systems Financing: The Path to Universal Coverage." World Health Organization. https://www.who.int/whr/2010/overview.pdf.

——_.2010b. Package of Essential Non-communicable Disease Interventions for Primary Health Care in Low Resource Settings. Geneva: WHO.

https://www.who.int/cardiovascular diseases/publications/pen2010/en/.

. 2013. "Roadmap. Strengthening People-Centred Health Systems in the WHO European Region. A Framework for Action towards Coordinated/Integrated Health Services Delivery (CIHSD)." WHO Regional Office for Europe, Copenhagen, Denmark. "'”

http://www.euro.who.int/ data/assets/pdf file/0005/231692/e96929-replacement-CIHSD-Roadmap171014b.pdf.

- - 2015. "People Centered and Integrated Health Services Overview. An Overview of the Evidence." https://www.who.int/servicedeliverysafety/areas/people-centred-care/evidence-overview/en/.

_-_. 2016a. Be Healthy Be Mobile Annual Report 2016. https://www.who.int/ncds/prevention/be-healthybe-mobile/report-2016/en/.

———.. 2016b. "Integrated Care Models: An Overview."

http://www.euro.who.int/ data/assets/pdf file/0005/322475/Integrated-care-models-overview.pdf.

———. 2016c. "Global Strategy on Human Resources for Health: Workforce 2030."

https://www.who.int/hrh/resources/global strategy workforce2030 14 print.pdf?ua=1. 
_- - 2017. "STEPwise Approach to Chronic Disease Risk Factor Surveillance." STEPS.

http://www.who.int/ncds/surveillance/steps/WHO Turkey Risk Factors A4 ENG 19.06.2018.pdf.

- - 2018. Final Report. WHO GCM/NCD Working Group on the Inclusion of NCDs in other Programmatic Areas (Working Group 3.1, 2016-2017). Geneva: WHO. https://apps.who.int/iris/handle/10665/312271

WHO Europe. 2015. "Ambulatory Care Sensitive Conditions in Germany."

http://www.euro.who.int/ data/assets/pdf file/0004/295573/ASCS-Germany-2015-rev1.pdf.

——_. 2016. "Ambulatory Care Sensitive Conditions in Portugal."

http://www.euro.who.int/ data/assets/pdf file/0007/307195/Ambulatory-care-sensitive-conditions-

Portugal.pdf.

WHO, MoH, and UNDP. 2018. "Prevention and Control of NCDs in Turkey, The Case for Investment."

http://www.euro.who.int/ data/assets/pdf file/0009/387162/bizzcase-tur-eng.pdf?ua=1.

WHO, OECD, and International Bank for Reconstruction and Development/World Bank. 2018. "Delivering Quality Health Services: A Global Imperative for Universal Health Coverage."

https://www.worldbank.org/en/topic/universalhealthcoverage/publication/delivering-quality-healthservices-a-global-imperative-for-universal-health-coverage.

Willis, C. D., J. Saul, H. Bevan, M. Scheirer, A. Best, T. Greenhalgh, R. Mannion, E. Cornelissen, D, Howland, E. Jenkins, and J. Bitz. 2016. "Sustaining Organizational Culture Change in Health Systems." Journal of Health Organization and Management 30 (1): 2-30. http://dx.doi.org/10.1108/JHOM-07-2014-0117.

Wootton, Richard. 2012. "Twenty Years of Telemedicine in Chronic Disease Management-An Evidence Synthesis. Journal of Telemedicine and Telecare 18: 211-220. doi:10.1258/jtt.2012.120219. 
The ER utilization in the biggest provinces of Turkey is in line with the countrywide utilization patterns. In all the six provinces, the majority of these cases have a triage category yellow or green and the high utilization of ER services mostly concentrate after business hours (particularly between 1600 and 2400 hours and during weekends).
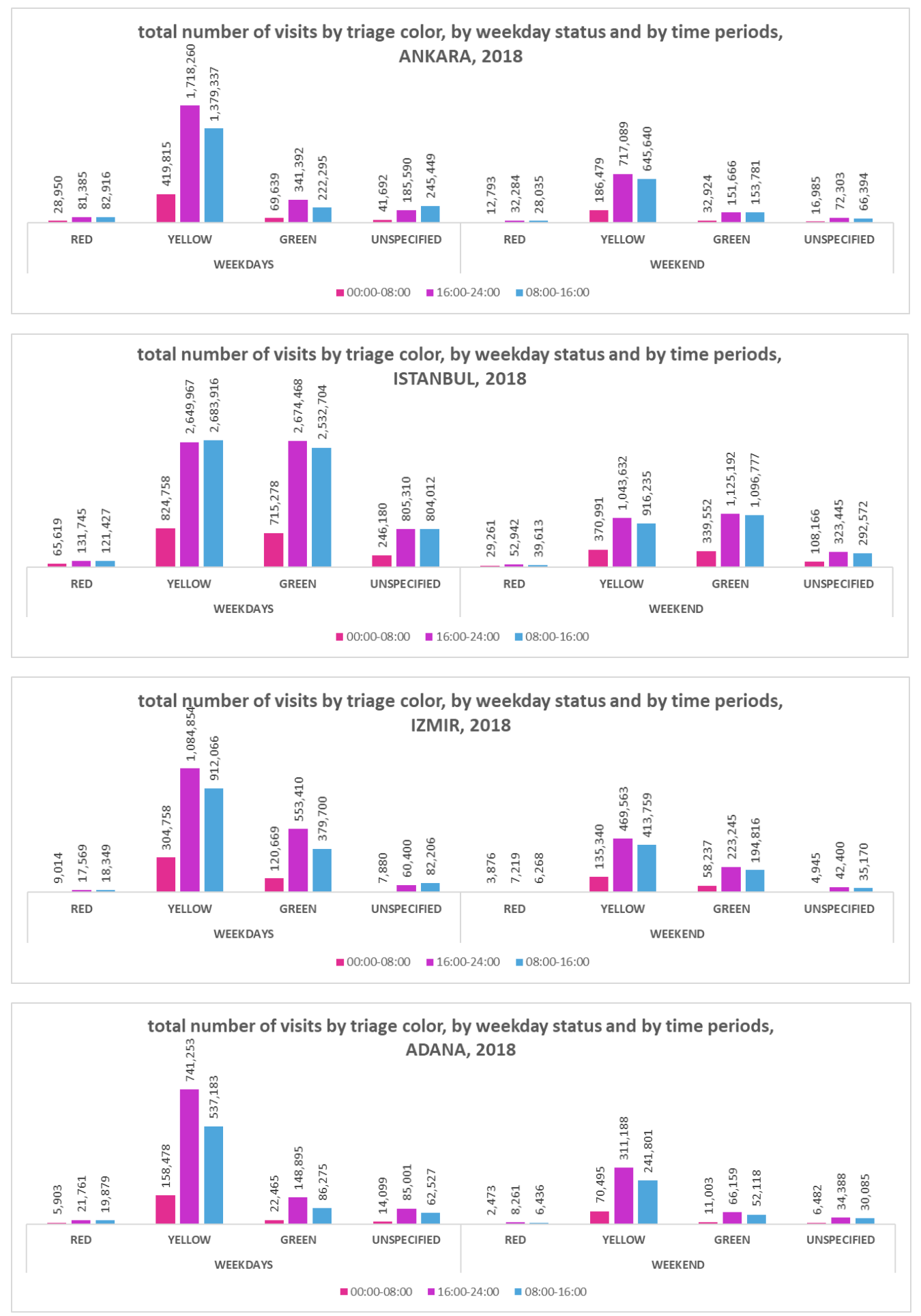

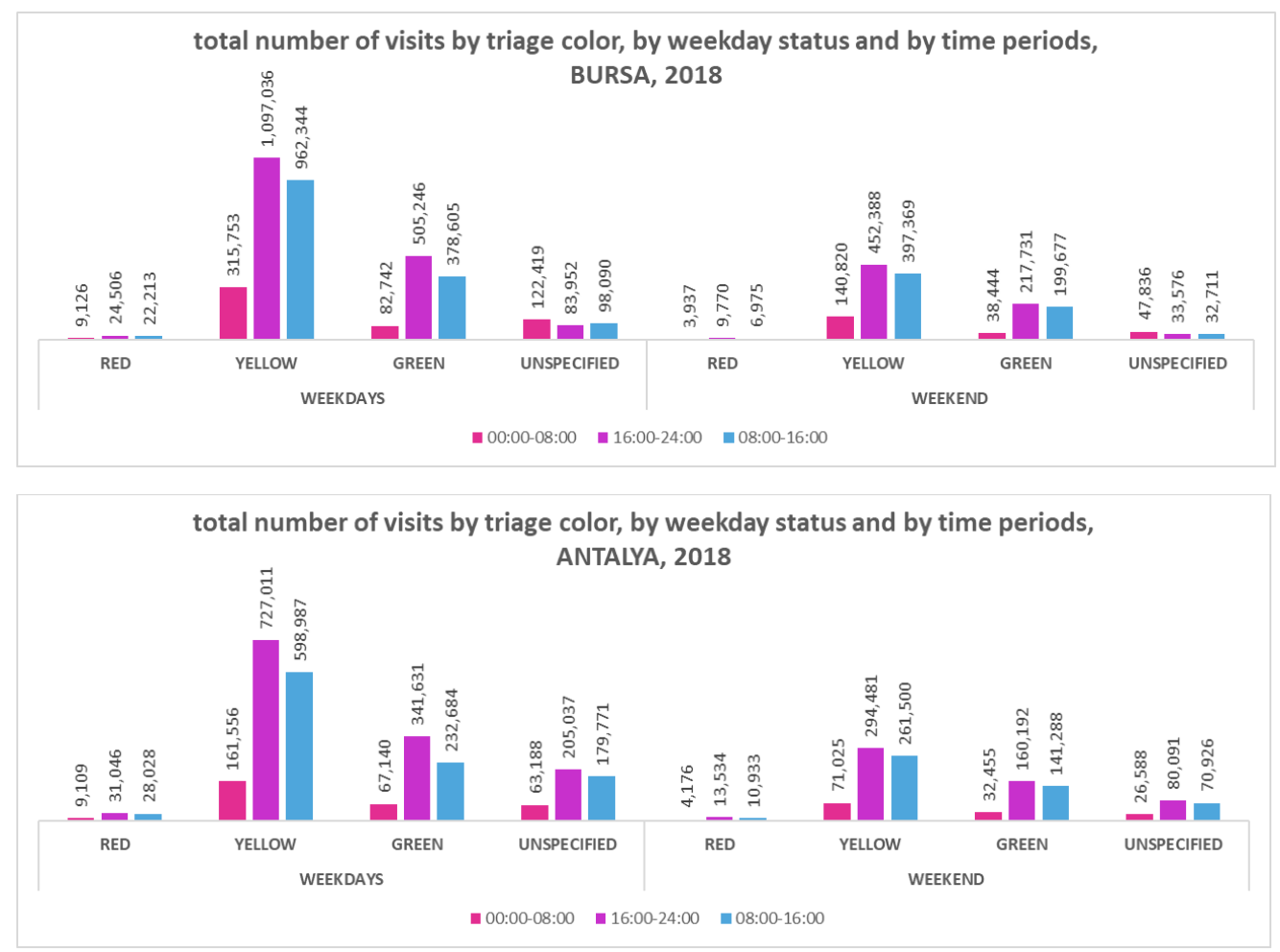


\section{ANNEX 2 ROLE OF PRIMARY CARE PROVIDERS IN NCD AND ELDERLY CARE AND PATIENT PATHWAYS DEVELOPED BY THE MOH FOR HT AND CVD RISK ASSESSMENT}

The following diagram shows the care provision tasks and the workflow of FMC and HLC staff. It also highlights the coordination between these two centers where HLCs take action either through group interventions or individual interventions.

\section{Role of FMCs and HLCs in NCD and elderly care}

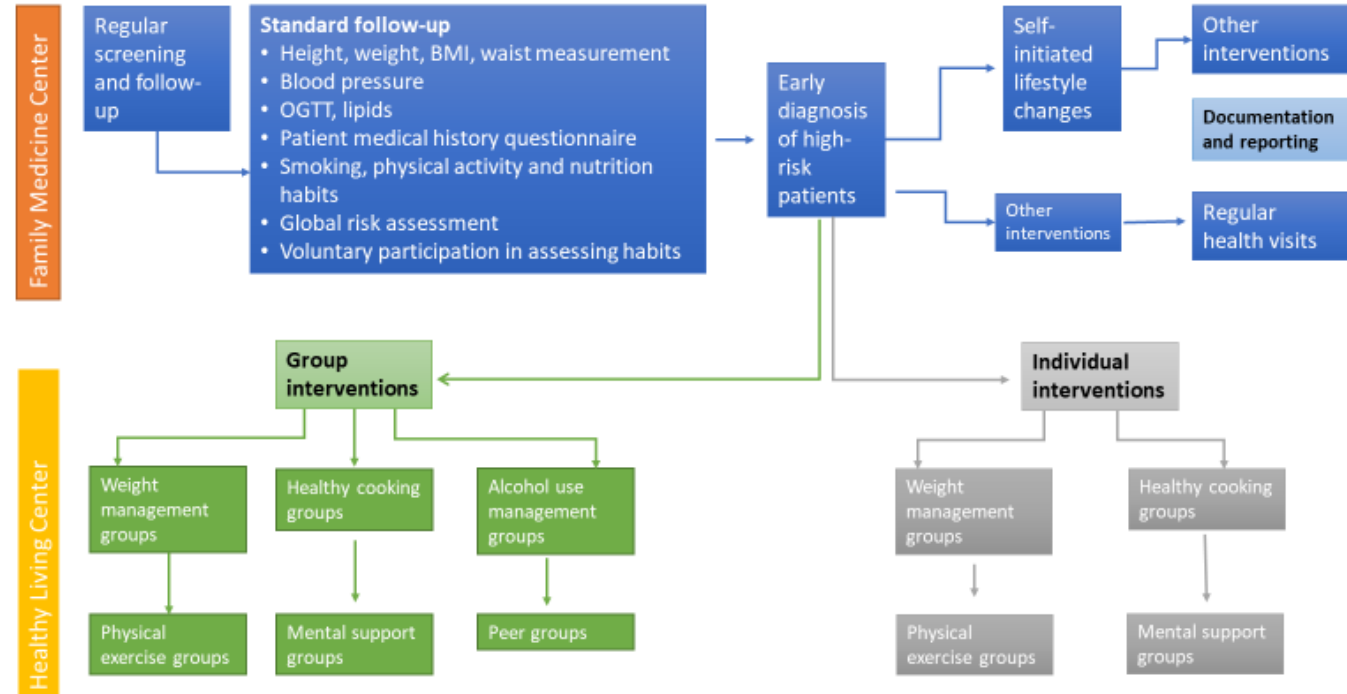

\section{Patient Pathways for HT}

The following diagrams show the HT management process flow for and the healthy lifestyle advice given to the patient. Lifestyle advice includes recommendations on dietary habits, physical activity, tobacco, and alcohol cessation.

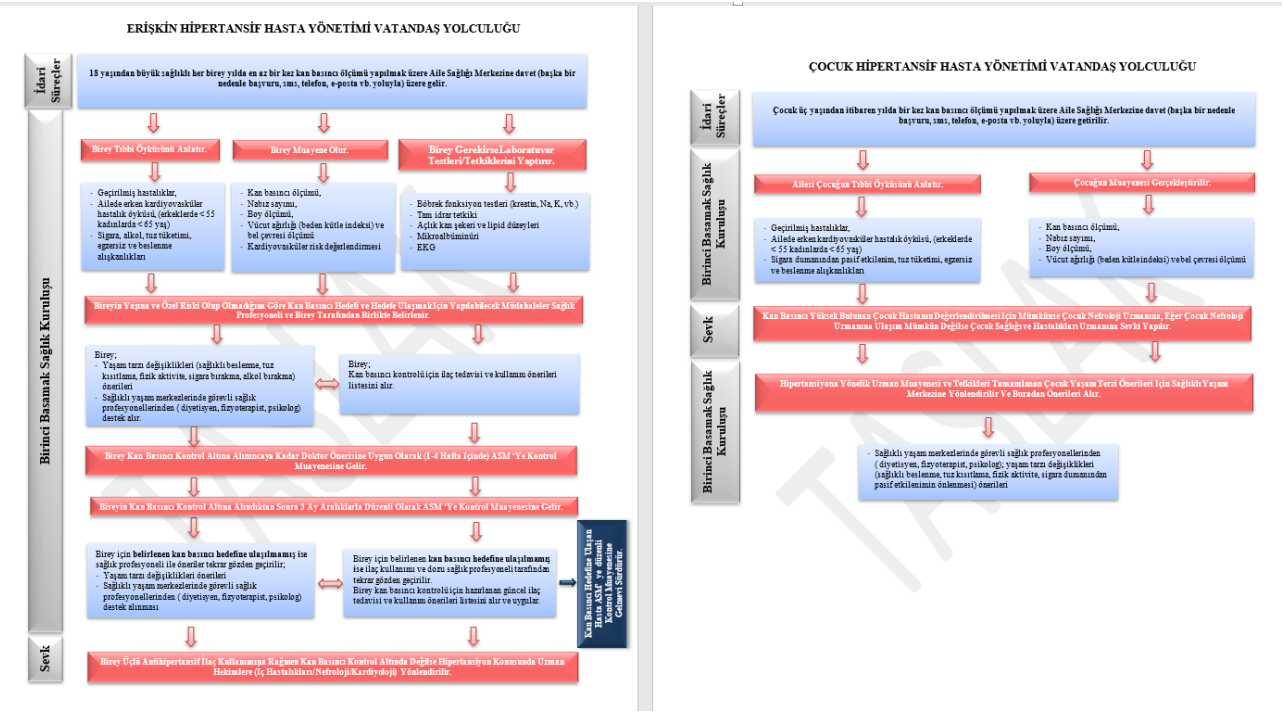




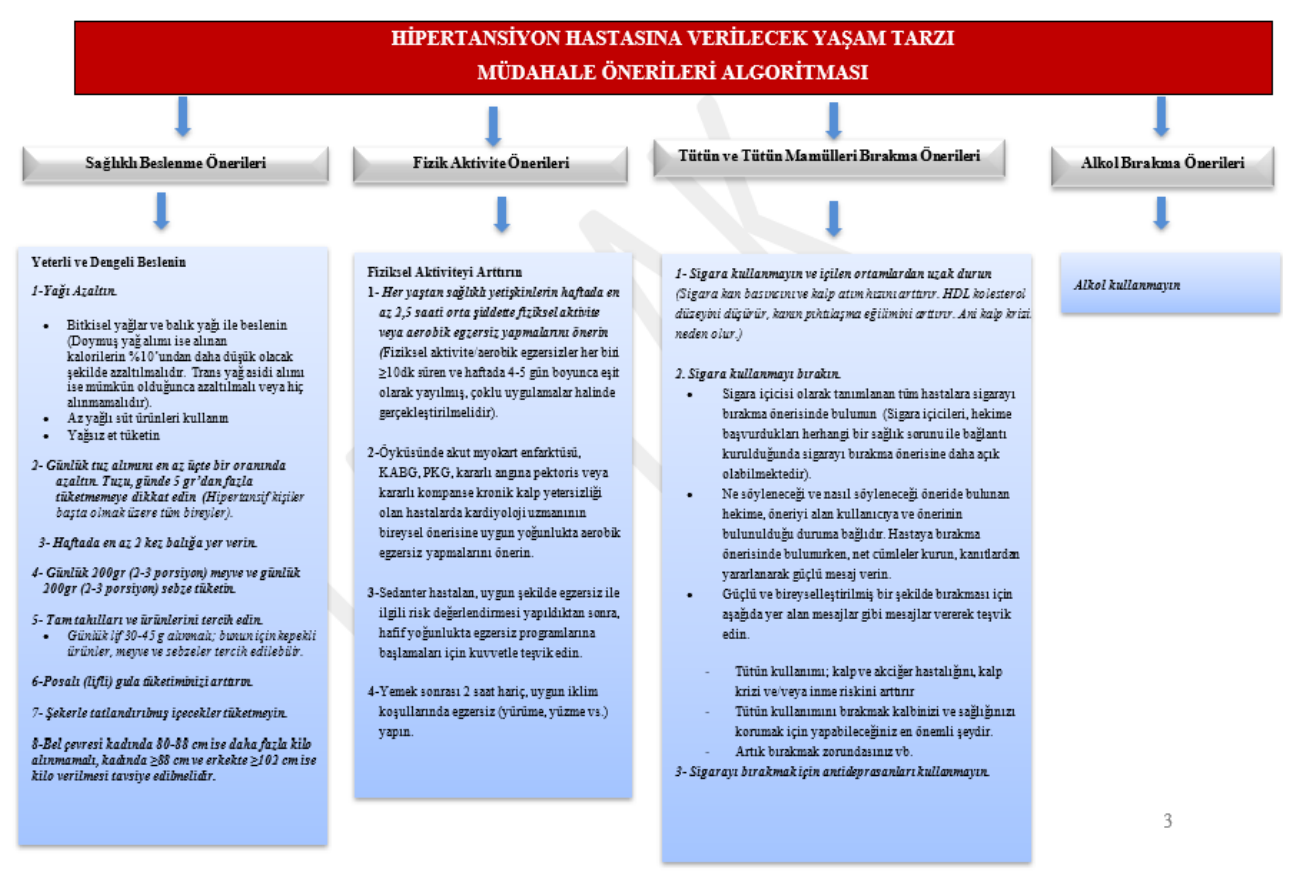

\section{Patient Pathways for CVD Risk Assessment}

The following diagrams show the CVD risk assessment process flow and the healthy lifestyle advice given to the patient. Lifestyle advice includes recommendations on dietary habits, physical activity, tobacco and alcohol cessation.

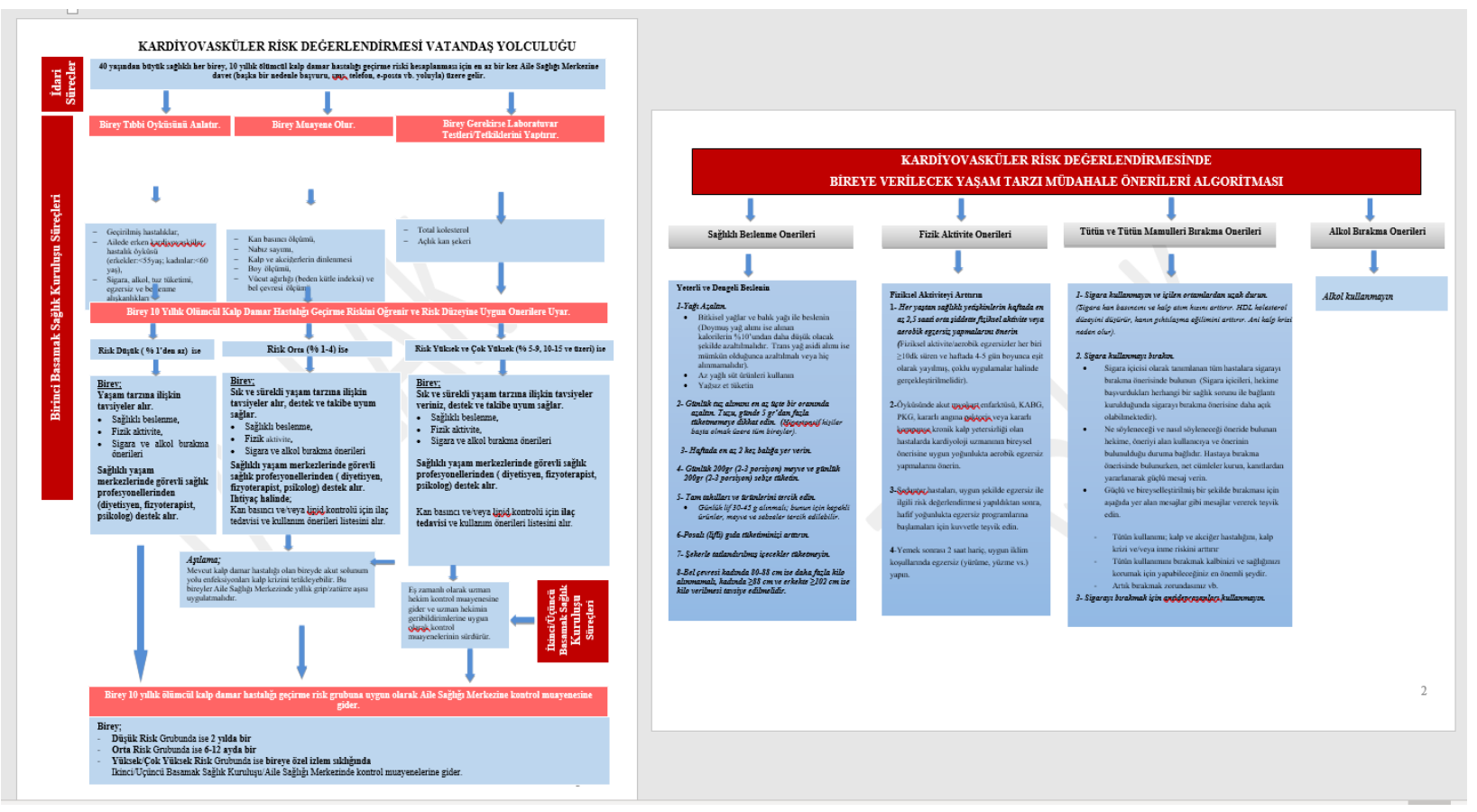

